\title{
Comprehensive Review of Radial Distribution Test Systems
}

\author{
Y. Latreche ${ }^{(1)}$, H.R.E.H. Bouchekara ${ }^{\left(2,{ }^{*}\right)}$, K. Naidu ${ }^{(3)}$, H. Mokhlis ${ }^{(4)}$, W.M. Dahalan ${ }^{(5)}$
}

(1) Modeling of Energy Renewable Devices and Nano-Metric (MoDERNa) Laboratory, University of Freres Mentouri Constantine, 25000 Constantine, Algeria.

(2) Department of Electrical Engineering, University of Hafr Al Batin, Hafr Al Batin 31991..

(3) Electrical Technology Section, University Kuala Lumpur British Malaysian Institute, Batu 8 Jalan Sungai Pusu, 53100 Gombak, Selangor, Malaysia

(4) Department of Electrical Engineering, Faculty of Engineering, University Malaya, 50603 Kuala Lumpur, Malaysia

(5) Department of Electrical Engineering, University of Kuala Lumpur, Malaysian Institute of Marine Engineering Technology, 32000, Perak, Malaysia

*Corresponding author: bouchekara.houssem@gmail.com, Tel: (+213)666605628, Fax: (+213)31908113

\begin{abstract}
:
This paper carries out a comprehensive review on the existing radial distribution test systems available for the power system distribution research area. The information available from this review can be used to establish a database of cases suitable for distribution operation and planning studies. The importance of distribution system, the structure of the system in the grid and the various configurations of the distribution network are provided. The major requirement of the distribution network is highlighted to underline important system parameters to be considered in design and planning stages. Various research can be conducted in the distribution network such as load flow algorithms, optimal incorporation of distributed generation, network reconfiguration, optimal capacitor allocation, etc. Important details pertaining to each test system such as bus and line data, network diagrams and power flow results are given. Additional information such as active and reactive loads and losses, minimum voltage values and bus location with weakest voltage values are given as well. Application of the reviewed works based on the test system is tabulated and presented. The information presented in this paper will be beneficial for future research work in the distribution system design and planning.
\end{abstract}

\section{Keywords:}

Education, radial systems, distribution, power systems, test systems. 


\section{Introduction}

Test systems are widely used in power system studies. Researchers often use these systems as benchmarks to implement their proposed algorithm to evaluate the algorithm's effectiveness and robustness. However, there are variation in the test systems despite its similar configuration. This is because some test systems are modified to suit the nature of the application. Changes in the line or bus data will significantly impact the behaviour and characteristics of the power system. In view of this, it is vital to have a proper database of the various distribution test system available with their pertinent network information to streamline research effort in this area.

The distribution network is often connected in radial connection. The radial nature of the distribution system is important due to its simplicity and least expensive configuration (Xavier and Llavall 2011). The laterals in the system is connected to a main source of power. Time grading based protection system can be implement to ensure that the tripping is done stage by stage for the downstream nodes. Various investigation based on planning and operation has been carried out for the distribution system. Hence it is important to ensure that a proper database comprising all these distribution networks are built. This is to ensure that researchers can easily benchmark the solution and verify their algorithm's effectiveness. In order to address this, this paper presents a comprehensive review on the most commonly used radial distribution test systems by giving network diagrams, respective network data and the power flow results. The main aim of this paper is to offer to the research community the possibility to investigate various test systems in the same paper for operation and planning studies on radial distribution systems.

In order to present the various distribution test system, the following information is provided:

- Network diagram: The connection diagram of the distribution system indicating the bus number and the loads connected in each bus.

- Network data: The line and bus data information ( $\mathrm{R}$ and $\mathrm{X}$ in $\mathrm{p} . \mathrm{u}$. .), the active and reactive power load and the $\mathrm{S}_{\text {base }}$ and $\mathrm{V}_{\text {base. }}$

- Load flow study: Total active power losses, Total reactive power losses and the bus where the minimum voltage is obtained

- The original reference for further information.

In this paper, the MATPOWER (Zimmerman et al. 2011) has been used to simulate the power flow studies using two methods namely the Newton Raphson (NR) and the Power Summation (PQSUM') algorithms. Moreover, a function that converts MATPOWER cases to VSOP (Gözel et al. 
2008) cases is developed. All the cods and/or the editable diagrams for the various test systems can be obtained through email by contacting the corresponding author.

The remaining paper is organized as following. In section 2, the important properties of the distribution system such as overview of the system and the network configuration is given. Section 3 provides the overview on distribution system which includes general review. The comprehensive database of the test system is given in Section 4. Application of reviewed works based on the test systems is given in Section 5 and the paper is concluded in Section 6.

\section{Overview on distribution systems}

There are many different voltage levels for each stage in the power system network. These voltage levels are required for different tasks in system. The ultra-high voltage grid is used to transmit power from large power plants over long distances. The high voltage grid is either connected to some large industries or stepped down through distribution substation to low voltage grids. In order to serve the customers / end users at the low voltage grid, the voltage is further stepped down to $230 / 400 \mathrm{~V}$. If the power requirement is high, large industries can be connected to the medium voltage grid. The general overview of this connection and the voltage levels is shown Figure 1 (Almasri 2011).

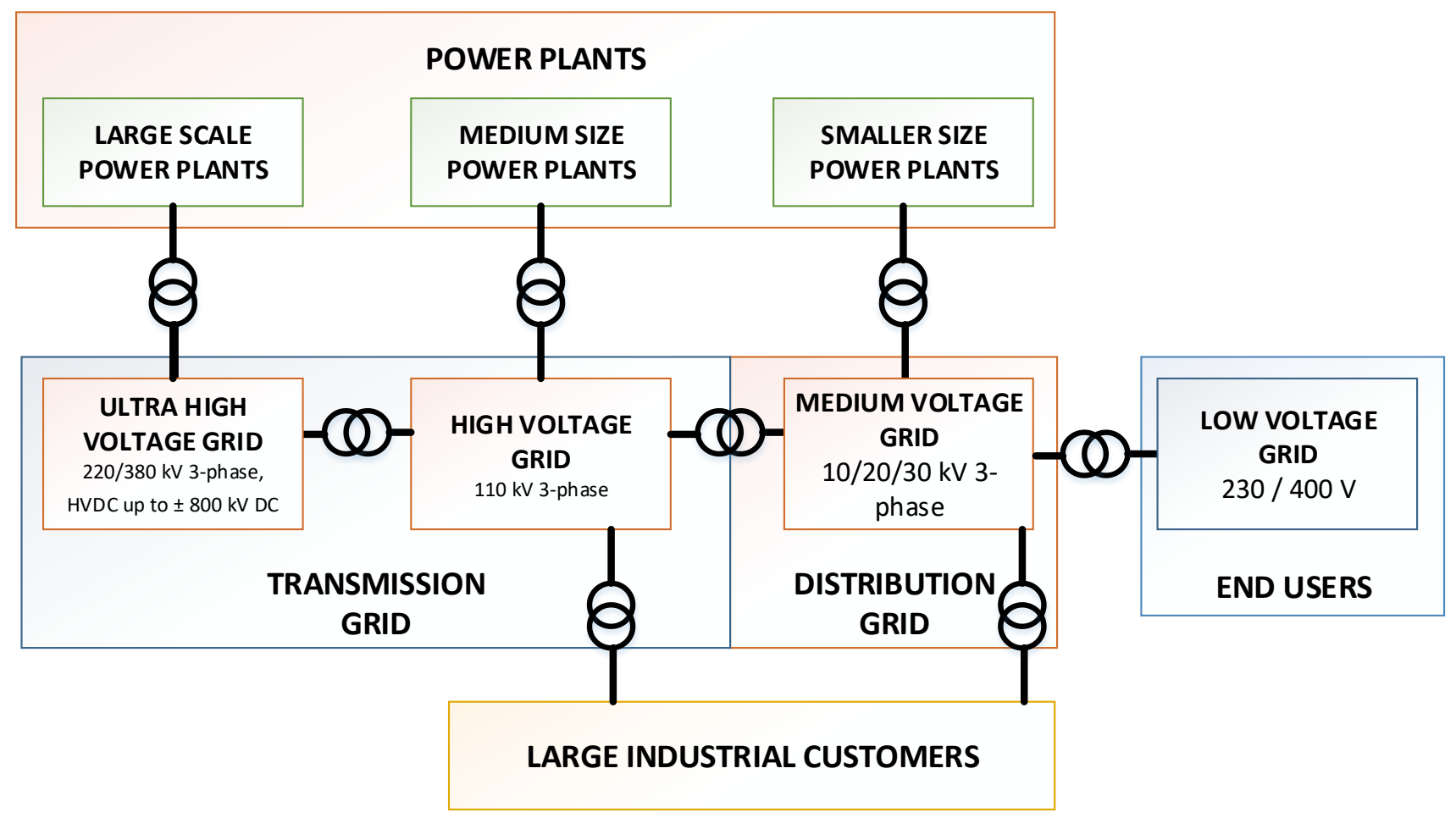

Figure 1 : General grid structure in the UCTE grid (UCTE - Union for the Co-ordination of Transmission of Electricity). 
The distribution system is defined as the portion of an electric system that delivers electric energy from transformation points on the transmission system to the customer (Warren 2003). The distribution system or network at it is termed comprises of feeders, distributors and service mains (V.K. Mehta and R. Mehta 2011). These three main components are responsible to deliver and distribute the power to end users.

- Feeders: The feeders are the main conduit in the substation which connects the substation (source of power) to the area where the power is required to be delivered. Usually no tappings are taken from the feeders. The endpoint of the feeders is connected to the distributors.

- Distributors: The tappings for the end users are taken from this point. The main consideration considered here is the voltage drop since various tapping will traduce voltage drop on the distributor.

- Service mains: Cables the connects from the distributors to the end users.

Figure 2 shows a general connection diagram of the typical low voltage distribution system which shows how the source of power (distribution substation) is delivered to the end users through the feeders, distributors and service means.

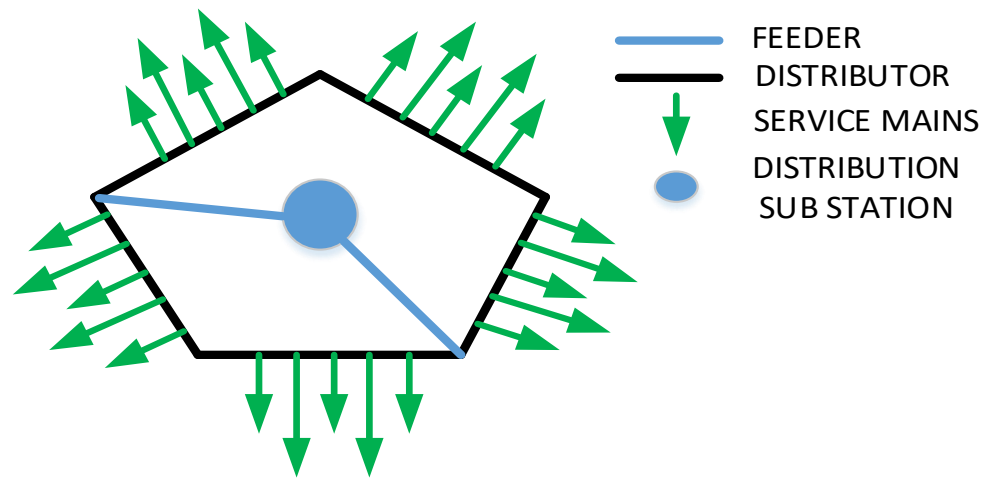

Figure 2 : General overview of low voltage distribution system.

The distribution system which is either connected using overhead or underground cable have different network configurations. The network configurations describe the connection between the loads and the power source. The three general configurations used in distribution network and their advantages and disadvantages are shown in Table 1 (V.K. Mehta and R. Mehta 2011). 
Table 1 : Common distribution systems network configurations.

\begin{tabular}{|c|c|c|}
\hline $\begin{array}{c}\text { Network } \\
\text { configuration }\end{array}$ & Network connection & Description \\
\hline $\begin{array}{l}\text { Radial } \\
\text { network }\end{array}$ & & $\begin{array}{l}\text { Also known as spur network } \\
\text { Advantages } \\
\text { Simple configuration since source is fed at one } \\
\text { end. } \\
\text { Easy protection coordination and network } \\
\text { monitoring } \\
\text { Investment cost is low (depending on size of } \\
\text { network) } \\
\text { Disadvantages } \\
\text { Voltage fluctuation for consumers at the end } \\
\text { of the distributor if the total connected load on } \\
\text { the distributor changes } \\
\text { Spur networks will cause end users to be } \\
\text { disconnected (no power supply) if fault occurs } \\
\text { on the feeder or distributor that they are } \\
\text { connected to. }\end{array}$ \\
\hline $\begin{array}{l}\text { Ringed } \\
\text { network }\end{array}$ & & $\begin{array}{l}\text { Advantages } \\
\text { Power is supplied form both ends - In case of } \\
\text { fault in the feeder, power can be supplied form } \\
\text { the other end, less voltage drops along the line } \\
\text { and less voltage fluctuation } \\
\text { Higher number of end users can be connected } \\
\text { to the system compared to radial network } \\
\text { Disadvantages } \\
\text { Higher cost of investment due to complex } \\
\text { protection coordination (line differential } \\
\text { protection) } \\
\text { Switching operation for fault localisation is } \\
\text { more complex }\end{array}$ \\
\hline $\begin{array}{l}\text { Meshed } \\
\text { network }\end{array}$ & & $\begin{array}{l}\text { Advantages } \\
\text { More than one source of power / substation is } \\
\text { connected - increases reliability } \\
\text { Increased efficiency - power can be supplied } \\
\text { from different generating stations during peak } \\
\text { load hours } \\
\text { Disadvantages } \\
\text { Complex protection coordination due to } \\
\text { power flow from different sources. }\end{array}$ \\
\hline
\end{tabular}

In this paper we are interested in radial distribution systems. 


\section{Overview on distribution studies}

RDS test systems can be used in distribution systems for various purposes and studies, some of them are listed in the following subsections.

\subsection{Load Flow Analysis}

Load flow analysis is considered basic analysis for both transmission and distribution systems. The analysis provides the voltage and phase angle of the bus in the network at the transmission or distribution level. From these parameters, other parameters such as power flow and power losses can be determined. It is applied for planning and design stages of distribution systems as well as during operational stage. Due to the different characteristic of transmission and distribution system, the load flow methods are formulated differently. For transmission system the well-known methods are Newton-Raphson method and Fast-Decoupled method. Meanwhile, Backward-Forward method regarded as the common method for distribution system. In distribution system, researchers uses the test systems in order to validate their new load flow algorithms.

\subsection{Planning of radial distribution systems}

In general distribution systems planning involve development and enhancement of the existing system. In the development stage, main consideration is on the minimizing the design cost of the system. In this stage, the overall design of the system such as location of substation (HV and MV), medium voltage (MV) feeders routing, load points and transformer capacity need to be determined with the objective of minimizing the cost. At the same time, technical constrains must be also considered such as maximum allowable voltage drops at load nodes and the maximum load carrying capacity of lines, at peak load. Meanwhile for existing systems, main consideration is to minimize the operation cost and maximize system reliability.

\subsection{Optimal conductor size selection}

One of the important aspects of designing cost-effective and reliable distribution systems is to determine optimal sizing of conductors for the network. Un-optimal selection of conductors will yield high power losses that translated into high operating cost. Therefore, the main objective of this study is to minimize the investment cost and the energy losses subject to feasible operation constraints. Several parameters need to be taken into account in the formulation of conductor optimal sizing such as conductor's economic life, discount rate, cable and installation costs and type of circuit (overhead or underground). This study is also required for reconductoring process when; (1) there are excessive 
power losses in the existing system, (2) the maximum current capacity of existing circuits is violated or (3) the voltage magnitudes in the EDS are lower than its minimum limit.

\subsection{Optimal siting and sizing of DG}

Penetration distribution generation (DG) in the distribution system has provided system operators a challenging task to maximize its potential benefits. Two major properties that's influences the DG's behaviour in the system are its sitting (location) and size. One of the major challenges in deciding the location of the DG will be type of DG. The main types of DGs available are the variable power DGs (intermittent sources) and DGs with predictable power output (Wang and Nehrir 2004). The variable power DGs such as wind and photovoltaic are limited by meteorological and geographic factors. The DGs with predictable power output such as fuel cells and microturbines are mobile and can be placed anywhere. The optimal DG placement (ODGP) is also a challenging problem with critical importance. This due to the fact that incorrect placement or location of DG might increase power losses in the system and operating cost (Georgilakis and Hatziargyriou 2013). An optimal placement of the DG will guarantee improved network performance, power quality and reliability of supply. Researchers have used various objective functions for the ODGP problem such as minimization of power loss, minimization of cost, minimization of voltage deviations, minimization of system average interruption duration index (SAIDI), maximization of profit and maximization of voltage limit loadability. The size of the DG is not decided based on power consumption in the area alone, but careful consideration has to be taken to ensure that total power loss of system (Lee and Park 2009). Load flow analysis such optimal power flow is used to find the optimal DG size which can meet the pre-defined criteria. Reliability analysis which comprises of reliability index calculation, restoration analysis, protection / coordination analysis, fault analysis, load flow analysis and load estimation also have to be carried out to determine the optimal DG sitting and size (Zhu et al. 2006).

\subsection{Optimal siting and sizing of capacitors}

Capacitors are energy storage device that is used for a variety of purpose in power system. One of the main usage of capacitors in distribution system is to compensate reactive power loss. High reactive power losses leads to many issue in the distribution network such as high system loss, high voltage drop and low power factor (Baran and Wu 1989b). Among the challenges faced in integrating the capacitor into distribution system is determining the location, the size, the type and the controls scheme of the capacitor. Incorrect sizing and location of the capacitor reduced system benefits, disturbances in voltage profile, increased power loss and unfavourable power factor (Diab et al. 2017). Objective functions such as minimization of total system losses, minimization of bus voltage violation 
and load balancing in feeders are considered in the capacitor sizing and location problem. The capacitor placement and sizing in the distribution network has to carefully carried out since the network comprises of linear and non-linear loads. In light of that, power quality constraints are included to ensure that issues such as harmonics occurrence is prevented. Current research has highlighted the importance of switching transient overvoltage of capacitor banks which is a critical power quality issue that would negatively impact the reliability of the distribution network (Javadi et al. 2017). Hence, the optimal sizing and location of the capacitor will guarantee lower system loss, greater system benefits, improved voltage profile and enhanced power quality (Masoum et al. 2004).

\subsection{Network reconfiguration}

Network reconfiguration for distribution system requires optimization techniques and is performed commonly to reduce the real power loss of the network. It also enhances the quality of the electrical power and improves the system reliability and security. Network reconfiguration involves the process of selection of the switches, which will determine the best combination set of switches to be open, in order to optimize the network system. The execution of the process of selection should fulfil the requirement of optimization and satisfying the operating constraints. Meanwhile, the application of reconfiguration network is much simpler and cost efficient compared to other techniques. In general, reconfiguration have two primary objectives which are to provide the maximum amount of electrical supply to the end customers and reconfigure the network system automatically as soon as the problems arise. 


\section{Test systems}

\subsection{0-bus radial system}

This system is composed of 10 buses and 9 branches as shown in Figure 3. The bus data, line data and load flow solution of this system are given in Table 2 and they are driven from (Baghzouz and Ertem 1990).

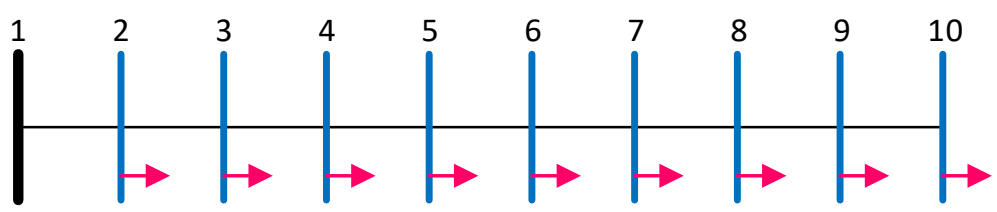

Figure 3 : Single line diagram of the 10-bus radial system.

Table 2: Data and load flow solution for the 10-bus radial system.

\begin{tabular}{|c|c|c|c|c|c|c|c|c|}
\hline $\begin{array}{c}\text { Branch } \\
\mathbf{N}^{\circ}\end{array}$ & $\begin{array}{c}\text { Bus } \\
\text { From }\end{array}$ & $\begin{array}{c}\text { Bus } \\
\text { To }\end{array}$ & $\begin{array}{c}\mathbf{R} \\
\text { (p.u.) }\end{array}$ & $\begin{array}{c}\mathbf{X} \\
\text { (p.u.) }\end{array}$ & $\begin{array}{c}\text { PL } \\
\text { (Bus To) } \\
\text { (kW) }\end{array}$ & $\begin{array}{c}\text { QL } \\
\text { (Bus To) } \\
\text { (kVAR) }\end{array}$ & $\begin{array}{c}\mathrm{V} \\
\text { (Bus To) } \\
\text { (pu) } \\
\text { NR } \\
\end{array}$ & $\begin{array}{c}\mathbf{V} \\
\text { (Bus To) } \\
\text { (pu) } \\
\text { PQSUM } \\
\end{array}$ \\
\hline 1 & 1 & 2 & 0.0233 & 0.0780 & 1840 & 460 & 0.9929 & 0.9929 \\
\hline 2 & 2 & 3 & 0.0026 & 0.1144 & 980 & 340 & 0.9874 & 0.9874 \\
\hline 3 & 3 & 4 & 0.1411 & 0.2278 & 1790 & 446 & 0.9634 & 0.9634 \\
\hline 4 & 4 & 5 & 0.1320 & 0.1150 & 1598 & 1840 & 0.9480 & 0.9480 \\
\hline 5 & 5 & 6 & 0.3749 & 0.3266 & 1610 & 600 & 0.9172 & 0.9172 \\
\hline 6 & 6 & 7 & 0.1711 & 0.1491 & 780 & 110 & 0.9072 & 0.9072 \\
\hline 7 & 7 & 8 & 0.3885 & 0.2200 & 1150 & 60 & 0.8890 & 0.8890 \\
\hline 8 & 8 & 9 & 0.9065 & 0.5134 & 980 & 130 & 0.8587 & 0.8587 \\
\hline 9 & 9 & 10 & 1.0101 & 0.5721 & 1640 & 200 & 0.8375 & 0.8375 \\
\hline
\end{tabular}

$\mathrm{S}_{\mathrm{base}}=100$ and $V_{\text {base }}=23 \mathrm{kV}$

The total active power load $=12368.0000 \mathrm{~kW}$

The total reactive power load $=4186.0000 \mathrm{kVAr}$

The total active power losses $=783.7754 \mathrm{~kW}$

The total reactive power losses $=1036.4710 \mathrm{kVAr}$

The minimum voltage value in p.u. $=0.838$

The minimum voltage is at bus 10 


\subsection{2-bus radial system}

This system is composed of 12 buses and 11 branches as shown in Figure 4. The bus data, line data and load flow solution of this system are given in Table 3 and they are driven from (Das 1994).

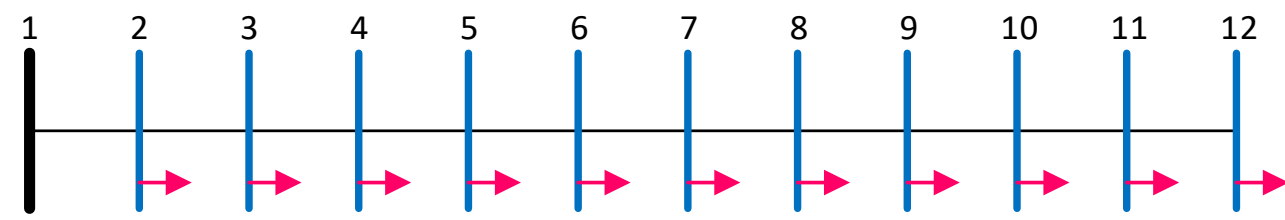

Figure 4 : Single line diagram of the 12-bus radial system.

Table 3 : Data and load flow solution for the 12-bus radial system.

\begin{tabular}{|c|c|c|c|c|c|c|c|c|}
\hline $\begin{array}{c}\text { Branch } \\
\mathbf{N}^{\circ}\end{array}$ & $\begin{array}{c}\text { Bus } \\
\text { From }\end{array}$ & $\begin{array}{c}\text { Bus } \\
\text { To }\end{array}$ & $\begin{array}{c}\mathbf{R} \\
(\mathbf{p . u .})\end{array}$ & $\begin{array}{c}\mathbf{X} \\
(\mathbf{p . u .})\end{array}$ & $\begin{array}{c}\text { PL } \\
(\mathbf{B u s} \text { To) } \\
(\mathbf{k W})\end{array}$ & $\begin{array}{c}\mathbf{Q L} \\
(\mathbf{B u s} \text { To) } \\
(\mathbf{k V A R})\end{array}$ & $\begin{array}{c}\mathbf{V} \\
(\mathbf{B u s} \text { To) } \\
(\mathbf{p u}) \\
\mathbf{\text { NR }}\end{array}$ & $\begin{array}{c}\mathbf{V} \\
(\mathbf{B u s} \text { To) } \\
(\mathbf{p u}) \\
\mathbf{\text { PQSUM }}\end{array}$ \\
\hline 1 & 1 & 2 & 0.9033 & 0.3760 & 60 & 60 & 0.9943 & 0.9943 \\
\hline 2 & 2 & 3 & 0.9785 & 0.4083 & 40 & 30 & 0.9890 & 0.9890 \\
\hline 3 & 3 & 4 & 1.7314 & 0.7215 & 55 & 55 & 0.9806 & 0.9806 \\
\hline 4 & 4 & 5 & 2.6347 & 1.0983 & 30 & 30 & 0.9698 & 0.9698 \\
\hline 5 & 5 & 6 & 0.9033 & 0.3760 & 20 & 15 & 0.9665 & 0.9665 \\
\hline 6 & 6 & 7 & 0.8281 & 0.3446 & 55 & 55 & 0.9637 & 0.9637 \\
\hline 7 & 7 & 8 & 3.6388 & 1.0041 & 45 & 45 & 0.9553 & 0.9553 \\
\hline 8 & 8 & 9 & 4.6628 & 1.3198 & 40 & 40 & 0.9473 & 0.9473 \\
\hline 9 & 9 & 10 & 2.3884 & 0.6760 & 35 & 30 & 0.9445 & 0.9445 \\
\hline 10 & 10 & 11 & 1.2512 & 0.3537 & 40 & 30 & 0.9436 & 0.9436 \\
\hline 11 & 11 & 12 & 1.0231 & 0.2901 & 15 & 15 & 0.9434 & 0.9434 \\
\hline
\end{tabular}

$S_{\text {base }}=100$ and $V_{\text {base }}=11 \mathrm{kV}$

The total active power load $=435.0000 \mathrm{~kW}$

The total reactive power load $=405.0000 \mathrm{kVAr}$

The total active power losses $=20.7135 \mathrm{~kW}$

The total reactive power losses $=8.0410 \mathrm{kVAr}$

The minimum voltage value in p.u. $=0.943$

The minimum voltage is at bus 12 


\subsection{5-bus radial system}

There are two 15-bus radial systems in the literature.

\subsubsection{System 1}

This system is composed of 15 buses and 14 branches as shown in Figure 5. The bus data, line data and load flow solution of this system are given in Table 4 and they are driven from (Das et al. 1995).

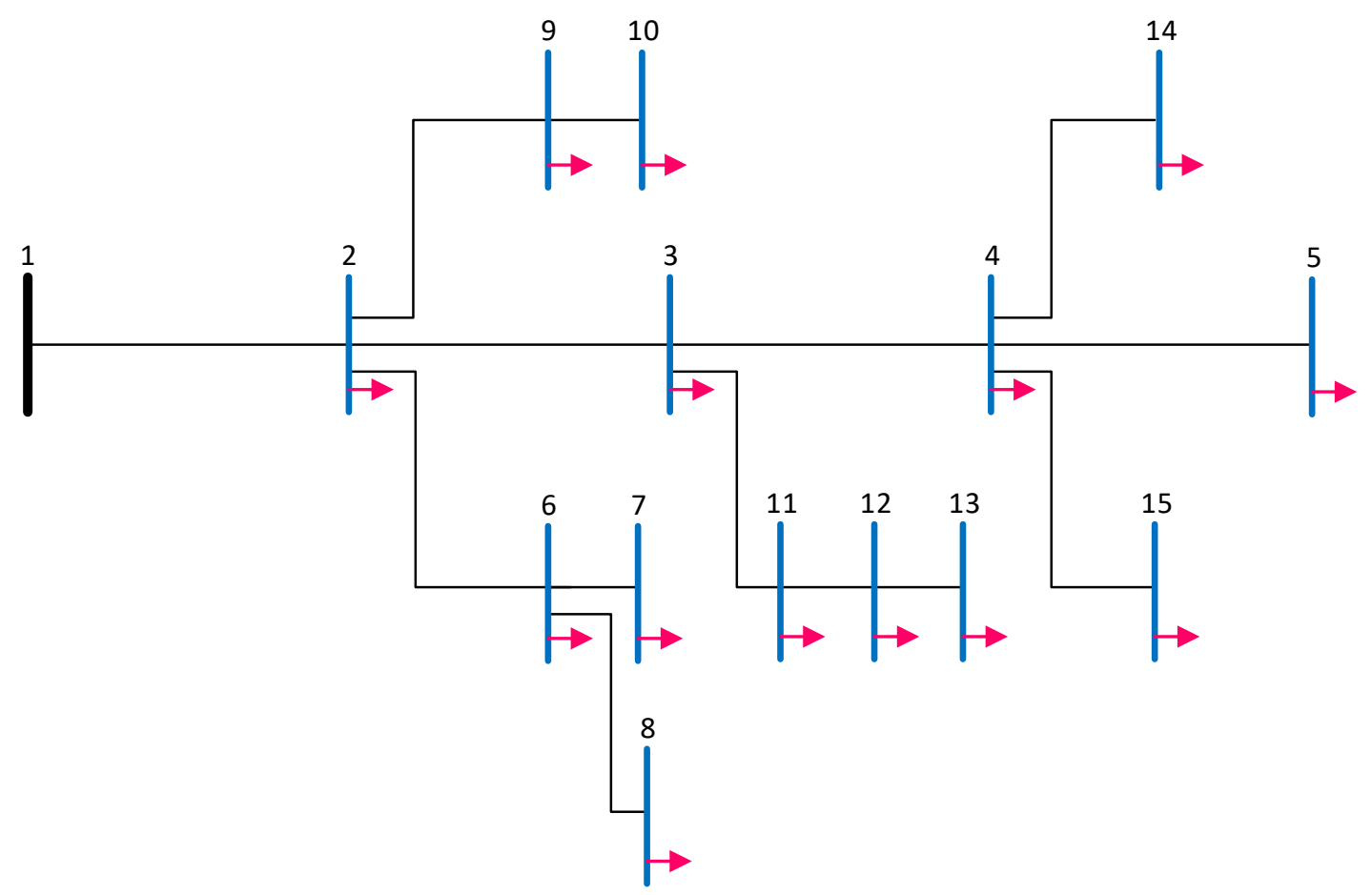

Figure 5 : Single line diagram of the 15-bus radial system.

Table 4 : Data and load flow solution for the 15-bus radial system.

\begin{tabular}{|c|c|c|c|c|c|c|c|c|}
\hline $\begin{array}{c}\text { Branch } \\
\mathbf{N}^{\circ}\end{array}$ & $\begin{array}{c}\text { Bus } \\
\text { From }\end{array}$ & $\begin{array}{c}\text { Bus } \\
\text { To }\end{array}$ & $\begin{array}{c}\mathbf{R} \\
(\mathbf{p . u} .)\end{array}$ & $\begin{array}{c}\mathbf{X} \\
(\mathbf{p . u .})\end{array}$ & $\begin{array}{c}\text { PL } \\
(\text { Bus To) } \\
(\mathbf{k W})\end{array}$ & $\begin{array}{c}\mathbf{Q L} \\
(\mathbf{B u s} \text { To) } \\
(\mathbf{k V A R})\end{array}$ & $\begin{array}{c}\mathbf{V} \\
(\mathbf{B u s} \text { To) } \\
(\mathbf{p u}) \\
\mathbf{N R}\end{array}$ & $\begin{array}{c}\mathbf{V} \\
(\mathbf{B u s} \text { To) } \\
(\mathbf{p u}) \\
\text { PQSUM }\end{array}$ \\
\hline 1 & 1 & 2 & 1.1183 & 1.0938 & 44.1 & 45.0 & 0.9713 & 0.9713 \\
\hline 2 & 2 & 3 & 0.9671 & 0.9460 & 70.0 & 71.4 & 0.9567 & 0.9567 \\
\hline 3 & 3 & 4 & 0.6951 & 0.6799 & 140.0 & 142.8 & 0.9509 & 0.9509 \\
\hline 4 & 4 & 5 & 1.2591 & 0.8493 & 44.1 & 45.0 & 0.9499 & 0.9499 \\
\hline 5 & 2 & 9 & 1.6638 & 1.1222 & 70.0 & 71.4 & 0.9680 & 0.9680 \\
\hline 6 & 9 & 10 & 1.3940 & 0.9402 & 44.1 & 45.0 & 0.9669 & 0.9669 \\
\hline 7 & 2 & 6 & 2.1134 & 1.4255 & 140.0 & 142.8 & 0.9582 & 0.9582 \\
\hline 8 & 6 & 7 & 0.8993 & 0.6066 & 140.0 & 142.8 & 0.9560 & 0.9560 \\
\hline 9 & 6 & 8 & 1.0342 & 0.6976 & 70.0 & 71.4 & 0.9570 & 0.9570 \\
\hline 10 & 3 & 11 & 1.4839 & 1.0009 & 140.0 & 142.8 & 0.9500 & 0.9500 \\
\hline 11 & 11 & 12 & 2.0235 & 1.3649 & 70.0 & 71.4 & 0.9458 & 0.9458 \\
\hline 12 & 12 & 13 & 1.6638 & 1.1222 & 44.1 & 45.0 & 0.9445 & 0.9445 \\
\hline 13 & 4 & 14 & 1.8436 & 1.2436 & 70.0 & 71.4 & 0.9486 & 0.9486 \\
\hline 14 & 4 & 15 & 0.9893 & 0.6673 & 140.0 & 142.8 & 0.9484 & 0.9484 \\
\hline
\end{tabular}


$\mathrm{S}_{\text {base }}=100$ and $V_{\text {base }}=11 \mathrm{kV}$

The total active power load $=1226.4000 \mathrm{~kW}$

The total reactive power load $=1251.1785 \mathrm{kVAr}$

The total active power losses $=61.4960 \mathrm{~kW}$

The total reactive power losses $=57.0393 \mathrm{kVAr}$

The minimum voltage value in p.u. $=0.945$

The minimum voltage is at bus 13

\subsubsection{System 2}

This second system is similar to the first one except for the values of $\mathrm{R}$ and $\mathrm{X}$ that are given in Table 5 (Battu et al. 2016).

Table 5 : Data and load flow solution for the 15-bus radial system.

\begin{tabular}{|c|c|c|c|c|c|c|c|c|}
\hline $\begin{array}{c}\text { Branch } \\
\mathbf{N}^{\circ}\end{array}$ & $\begin{array}{c}\text { Bus } \\
\text { From }\end{array}$ & $\begin{array}{c}\text { Bus } \\
\text { To }\end{array}$ & $\begin{array}{c}\mathbf{R} \\
(\mathbf{p . u} .)\end{array}$ & $\begin{array}{c}\mathbf{X} \\
(\mathbf{p . u .})\end{array}$ & $\begin{array}{c}\mathbf{P L} \\
(\mathbf{B u s} \text { To) } \\
(\mathbf{k W})\end{array}$ & $\begin{array}{c}\mathbf{Q L} \\
(\mathbf{B u s} \text { To) } \\
(\mathbf{k V A R})\end{array}$ & $\begin{array}{c}\mathbf{V} \\
(\mathbf{B u s} \mathbf{T o}) \\
(\mathbf{p u}) \\
\mathbf{N R}\end{array}$ & $\begin{array}{c}\mathbf{V} \\
(\mathbf{B u s} \text { To) } \\
(\mathbf{p u}) \\
\mathbf{P Q S U M}\end{array}$ \\
\hline 1 & 1 & 2 & 0.7766 & 0.7596 & 44.1 & 45.0 & 0.9804 & 0.9804 \\
\hline 2 & 2 & 3 & 0.6716 & 0.6569 & 70.0 & 71.4 & 0.9704 & 0.9704 \\
\hline 3 & 3 & 4 & 0.4827 & 0.4722 & 140.0 & 142.8 & 0.9664 & 0.9664 \\
\hline 4 & 4 & 5 & 0.8744 & 0.5898 & 44.1 & 45.0 & 0.9658 & 0.9658 \\
\hline 5 & 2 & 9 & 1.1554 & 0.7793 & 70.0 & 71.4 & 0.9781 & 0.9781 \\
\hline 6 & 9 & 10 & 0.9680 & 0.6529 & 44.1 & 45.0 & 0.9773 & 0.9773 \\
\hline 7 & 2 & 6 & 1.4677 & 0.9900 & 140.0 & 142.8 & 0.9714 & 0.9714 \\
\hline 8 & 6 & 7 & 0.6245 & 0.4213 & 140.0 & 142.8 & 0.9699 & 0.9699 \\
\hline 9 & 6 & 8 & 0.7182 & 0.4844 & 70.0 & 71.4 & 0.9705 & 0.9705 \\
\hline 10 & 3 & 11 & 1.0305 & 0.6951 & 140.0 & 142.8 & 0.9658 & 0.9658 \\
\hline 11 & 11 & 12 & 1.4052 & 0.9478 & 70.0 & 71.4 & 0.9630 & 0.9630 \\
\hline 12 & 12 & 13 & 1.1554 & 0.7793 & 44.1 & 45.0 & 0.9621 & 0.9621 \\
\hline 13 & 4 & 14 & 1.2803 & 0.8636 & 70.0 & 71.4 & 0.9649 & 0.9649 \\
\hline 14 & 4 & 15 & 0.6870 & 0.4634 & 140.0 & 142.8 & 0.9648 & 0.9648 \\
\hline
\end{tabular}

$S_{\text {base }}=100$ and $V_{\text {base }}=11 \mathrm{kV}$

The total active power load $=1226.4000 \mathrm{~kW}$

The total reactive power load $=1251.1785 \mathrm{kVAr}$

The total active power losses $=41.4752 \mathrm{~kW}$

The total reactive power losses $=38.4636 \mathrm{kVAr}$

The minimum voltage value in p.u. $=0.962$

The minimum voltage is at bus 13 


\subsection{6-bus radial system}

The initial version of this system is composed of 3 feeders, 16 buses and 13 branches as shown in Figure 6. The bus data, line data and load flow solution of this system are given in Table 6 and they are driven from (Civanlar et al. 1988) and (Zhu 2002).
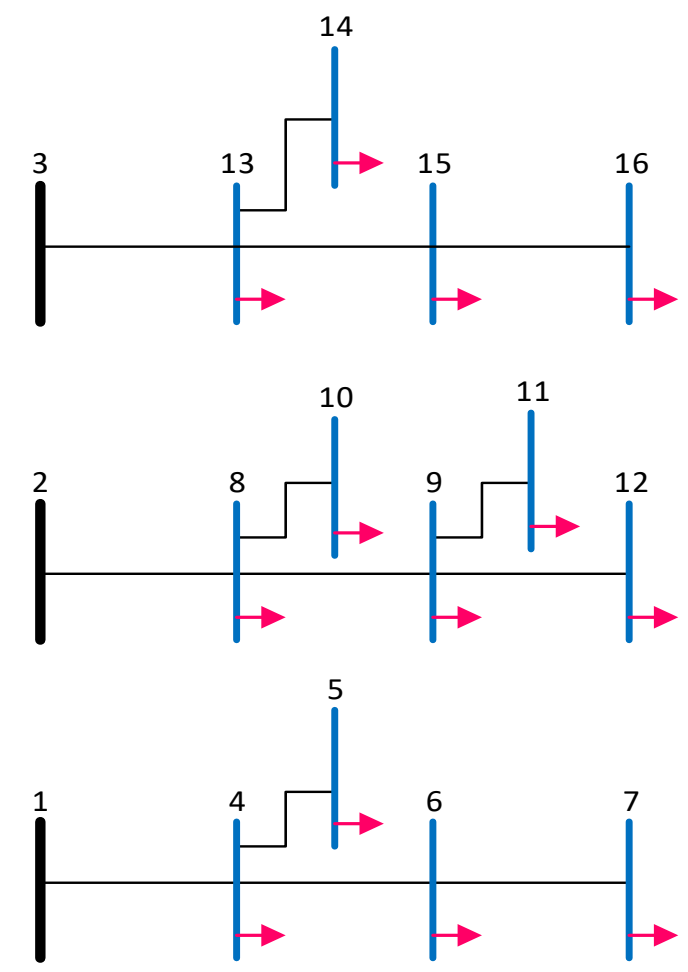

Figure 6 : Single line diagram of the 16-bus radial system.

Table 6 : Data and load flow solution for the 16-bus radial system.

\begin{tabular}{|c|c|c|c|c|c|c|c|c|}
\hline $\begin{array}{c}\text { Branch } \\
\mathbf{N}^{\circ}\end{array}$ & $\begin{array}{c}\text { Bus } \\
\text { From }\end{array}$ & $\begin{array}{c}\text { Bus } \\
\text { To }\end{array}$ & $\begin{array}{c}\mathbf{R} \\
(\mathbf{p . u} .)\end{array}$ & $\begin{array}{c}\mathbf{X} \\
(\mathbf{p . u .})\end{array}$ & $\begin{array}{c}\mathbf{P L} \\
(\mathbf{B u s} \text { To) } \\
(\mathbf{k W})\end{array}$ & $\begin{array}{c}\mathbf{Q L} \\
(\mathbf{B u s} \text { To) } \\
(\mathbf{k V A R})\end{array}$ & $\begin{array}{c}\mathbf{V} \\
(\mathbf{B u s} \text { To) } \\
(\mathbf{p u}) \\
\mathbf{\text { NR }}\end{array}$ & $\begin{array}{c}\mathbf{V} \\
(\mathbf{B u s} \text { To) } \\
(\mathbf{p u}) \\
\mathbf{P Q S U M}\end{array}$ \\
\hline 1 & 1 & 4 & 0.0468 & 0.0624 & 2000.0 & 1600.0 & 0.9942 & $/$ \\
\hline 2 & 4 & 5 & 0.0499 & 0.0686 & 3000.0 & 400.0 & 0.9924 & $/$ \\
\hline 3 & 4 & 6 & 0.0562 & 0.1123 & 2000.0 & -400.0 & 0.9913 & $/$ \\
\hline 4 & 6 & 7 & 0.0250 & 0.0250 & 1500.0 & 1200.0 & 0.9906 & $/$ \\
\hline 5 & 2 & 8 & 0.0686 & 0.0686 & 4000.0 & 2700.0 & 0.9871 & $/$ \\
\hline 6 & 8 & 9 & 0.0499 & 0.0686 & 5000.0 & 1800.0 & 0.9822 & $/$ \\
\hline 7 & 8 & 10 & 0.0686 & 0.0686 & 1000.0 & 900.0 & 0.9858 & $/$ \\
\hline 8 & 9 & 11 & 0.0686 & 0.0686 & 600.0 & -500.0 & 0.9822 & $/$ \\
\hline 9 & 9 & 12 & 0.0499 & 0.0686 & 4500.0 & -1700.0 & 0.9811 & $/$ \\
\hline 10 & 3 & 13 & 0.0686 & 0.0686 & 1000.0 & 900.0 & 0.9965 & $/$ \\
\hline 11 & 13 & 14 & 0.0562 & 0.0749 & 1000.0 & -1100.0 & 0.9968 & $/$ \\
\hline 12 & 13 & 15 & 0.0499 & 0.0686 & 1000.0 & 900.0 & 0.9949 & $/$ \\
\hline 13 & 15 & 16 & 0.0250 & 0.0250 & 2100.0 & -800.0 & 0.9946 & $/$ \\
\hline
\end{tabular}


$\mathrm{S}_{\text {base }}=100$ and $V_{\text {base }}=12.66 \mathrm{kV}$

Total active power load $=28700.0000 \mathrm{~kW}$

Total reactive power load $=5900.0000 \mathrm{kVAr}$

The total active power losses $=312.7765 \mathrm{~kW}$

The total reactive power losses $=361.1848 \mathrm{kVAr}$

The minimum voltage value in p.u. $=0.981$

The minimum voltage is at bus 12

However, in (Aman et al. 2014) the initial system has been transformed to a single feeder, 15bus radial distribution test system as shown in Figure 7. The bus data, line data and load flow solution of this system are given in Table 7.

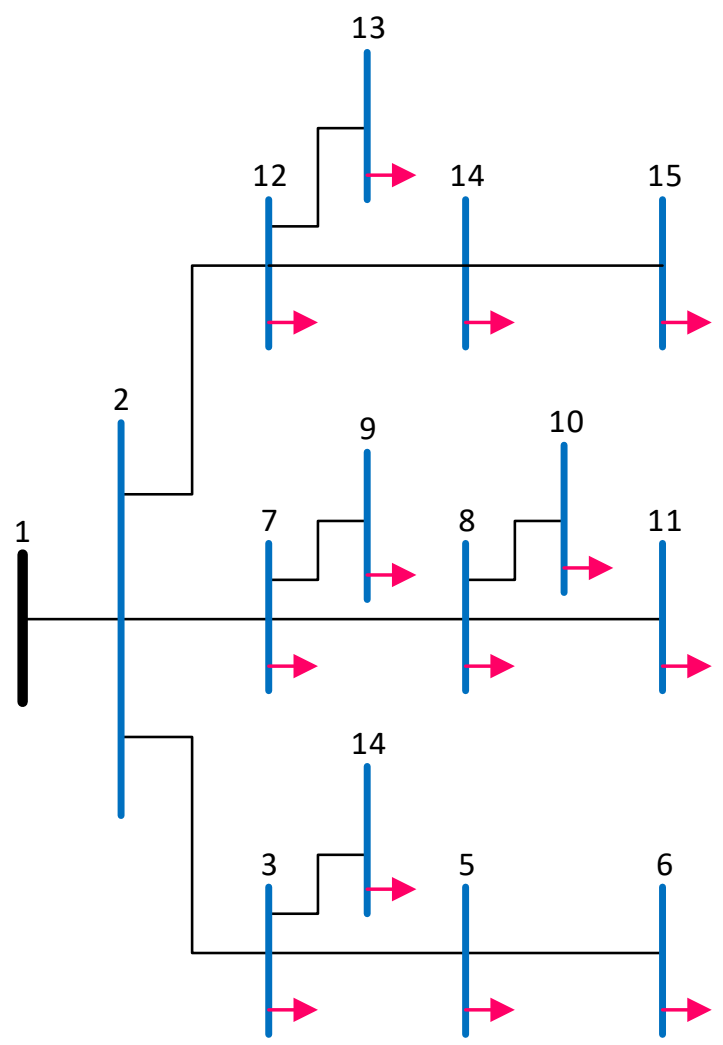

Figure 7 : Single line diagram of the 16-bus radial system. 
Table 7 : Data and load flow solution for the 16-bus radial system.

\begin{tabular}{|c|c|c|c|c|c|c|c|c|}
\hline $\begin{array}{c}\text { Branch } \\
\mathbf{N}^{\circ}\end{array}$ & $\begin{array}{c}\text { Bus } \\
\text { From }\end{array}$ & $\begin{array}{c}\text { Bus } \\
\text { To }\end{array}$ & $\begin{array}{c}\mathbf{R} \\
\text { (p.u.) }\end{array}$ & $\begin{array}{c}\mathbf{X} \\
\text { (p.u.) }\end{array}$ & $\begin{array}{c}\text { PL } \\
\text { (Bus To) } \\
\text { (kW) }\end{array}$ & $\begin{array}{c}\text { QL } \\
\text { (Bus To) } \\
\text { (kVAR) }\end{array}$ & $\begin{array}{c}\mathbf{V} \\
\text { (Bus To) } \\
\text { (pu) } \\
\text { NR } \\
\end{array}$ & $\begin{array}{c}\mathbf{V} \\
\text { (Bus To) } \\
\text { (pu) } \\
\text { PQSUM } \\
\end{array}$ \\
\hline 1 & 1 & 2 & 0.0000 & 0.0000 & 0.0 & 0.0 & 1.0000 & 1.0000 \\
\hline 2 & 2 & 3 & 0.0750 & 0.1000 & 2000.0 & 1600.0 & 0.9907 & 0.9907 \\
\hline 3 & 3 & 4 & 0.0800 & 0.1100 & 3000.0 & 400.0 & 0.9878 & 0.9878 \\
\hline 4 & 3 & 5 & 0.0900 & 0.1800 & 2000.0 & -400.0 & 0.9860 & 0.9860 \\
\hline 5 & 5 & 6 & 0.0400 & 0.0400 & 1500.0 & 1200.0 & 0.9849 & 0.9849 \\
\hline 6 & 2 & 7 & 0.1100 & 0.1100 & 4000.0 & 2700.0 & 0.9791 & 0.9791 \\
\hline 7 & 7 & 8 & 0.0800 & 0.1100 & 5000.0 & 1800.0 & 0.9711 & 0.9711 \\
\hline 8 & 7 & 9 & 0.1100 & 0.1100 & 1000.0 & 900.0 & 0.9769 & 0.9769 \\
\hline 9 & 8 & 10 & 0.1100 & 0.1100 & 600.0 & -500.0 & 0.9710 & 0.9710 \\
\hline 10 & 8 & 11 & 0.0800 & 0.1100 & 4500.0 & -1700.0 & 0.9693 & 0.9693 \\
\hline 11 & 2 & 12 & 0.1100 & 0.1100 & 1000.0 & 900.0 & 0.9944 & 0.9944 \\
\hline 12 & 12 & 13 & 0.0900 & 0.1200 & 1000.0 & -1100.0 & 0.9948 & 0.9948 \\
\hline 13 & 12 & 14 & 0.0800 & 0.1100 & 1000.0 & 900.0 & 0.9918 & 0.9918 \\
\hline 14 & 14 & 15 & 0.0400 & 0.0400 & 2100.0 & -800.0 & 0.9913 & 0.9913 \\
\hline
\end{tabular}

$\mathrm{S}_{\text {base }}=100$ and $V_{\text {base }}=12.66 \mathrm{kV}$

The total active power load $=28700.0000 \mathrm{~kW}$

The total reactive power load $=5900.0000 \mathrm{kVAr}$

The total active power losses $=511.4004 \mathrm{~kW}$

The total reactive power losses $=590.3684 \mathrm{kVAr}$

The minimum voltage value in p.u. $=0.969$

The minimum voltage is at bus 11 


\subsection{7-bus radial system}

This system is composed of 17 buses and 16 branches as shown in Figure 8. The bus data, line data and load flow solution of this system are given in Table 8 and they are driven from (Mendoza et al. 2007).

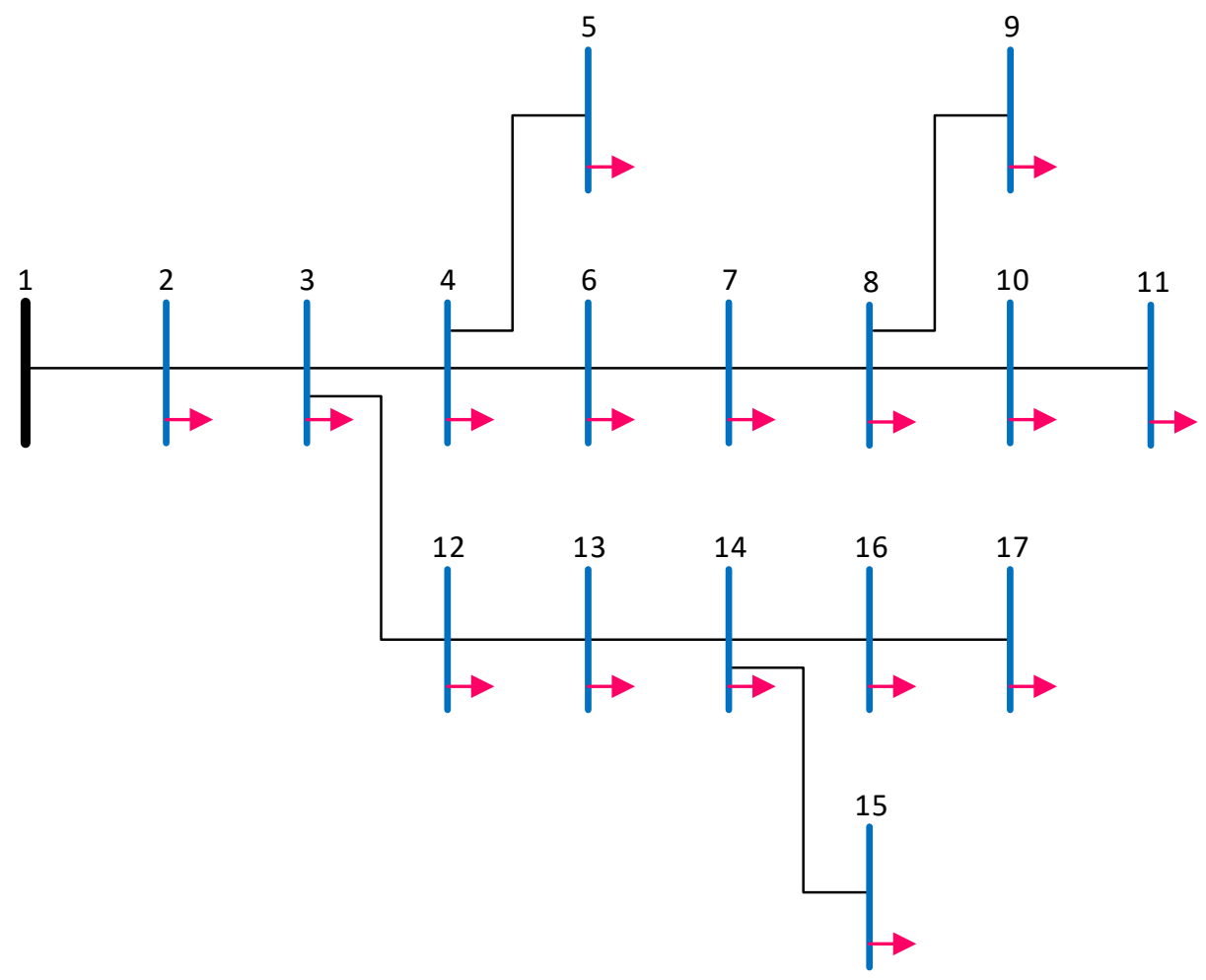

Figure 8 : Single line diagram of the 17-bus radial system.

Table 8 : Data and load flow solution for the 17-bus radial system.

\begin{tabular}{|c|c|c|c|c|c|c|c|c|}
\hline $\begin{array}{c}\text { Branch } \\
\mathbf{N}^{\circ}\end{array}$ & $\begin{array}{c}\text { Bus } \\
\text { From }\end{array}$ & $\begin{array}{c}\text { Bus } \\
\text { To }\end{array}$ & $\begin{array}{c}\mathbf{R} \\
(\mathbf{p . u .})\end{array}$ & $\begin{array}{c}\mathbf{X} \\
(\mathbf{p . u .})\end{array}$ & $\begin{array}{c}\text { PL } \\
(\mathbf{B u s} \text { To) } \\
(\mathbf{k W})\end{array}$ & $\begin{array}{c}\text { QL } \\
(\mathbf{B u s} \text { To) } \\
(\mathbf{k V A R})\end{array}$ & $\begin{array}{c}\mathbf{V} \\
(\mathbf{B u s} \text { To) } \\
(\mathbf{p u}) \\
\mathbf{\text { NR }}\end{array}$ & $\begin{array}{c}\mathbf{V} \\
(\mathbf{B u s} \text { To) } \\
(\mathbf{p u}) \\
\mathbf{\text { PQSUM }}\end{array}$ \\
\hline 1 & 1 & 2 & 0.0500 & 0.0500 & 800.00 & 600.00 & 0.9894 & 0.9894 \\
\hline 2 & 2 & 3 & 0.1100 & 0.1100 & 800.00 & 600.00 & 0.9678 & 0.9678 \\
\hline 3 & 3 & 4 & 0.1500 & 0.1100 & 800.00 & 600.00 & 0.9496 & 0.9496 \\
\hline 4 & 4 & 5 & 0.0800 & 0.1100 & 800.00 & 640.00 & 0.9482 & 0.9482 \\
\hline 5 & 4 & 6 & 0.1100 & 0.1100 & 1200.00 & 160.00 & 0.9383 & 0.9383 \\
\hline 6 & 6 & 7 & 0.0400 & 0.0400 & 800.00 & -160.00 & 0.9347 & 0.9347 \\
\hline 7 & 7 & 8 & 0.8000 & 0.1100 & 600.00 & 480.00 & 0.8898 & 0.8898 \\
\hline 8 & 8 & 9 & 0.0750 & 0.1000 & 1600.00 & 1080.00 & 0.8872 & 0.8872 \\
\hline 9 & 8 & 10 & 0.0900 & 0.1800 & 2000.00 & 720.00 & 0.8852 & 0.8852 \\
\hline 10 & 10 & 11 & 0.0400 & 0.0400 & 400.00 & 360.00 & 0.8848 & 0.8848 \\
\hline 11 & 3 & 12 & 0.1100 & 0.1100 & 240.00 & -200.00 & 0.9625 & 0.9625 \\
\hline 12 & 12 & 13 & 0.0400 & 0.0400 & 1800.00 & 800.00 & 0.9606 & 0.9606 \\
\hline 13 & 13 & 14 & 0.0900 & 0.1200 & 400.00 & 360.00 & 0.9587 & 0.9587 \\
\hline 14 & 14 & 15 & 0.1100 & 0.1100 & 400.00 & -440.00 & 0.9587 & 0.9587 \\
\hline 15 & 14 & 16 & 0.0800 & 0.1100 & 400.00 & 360.00 & 0.9576 & 0.9576 \\
\hline 16 & 16 & 17 & 0.0400 & 0.0400 & 840.00 & -320.00 & 0.9574 & 0.9574 \\
\hline
\end{tabular}


$\mathrm{S}_{\text {base }}=100$ and $V_{\text {base }}=23 \mathrm{kV}$

The total active power load $=13880.0000 \mathrm{~kW}$

The total reactive power load $=5640.0000 \mathrm{kVAr}$

The total active power losses $=950.6771 \mathrm{~kW}$

The total reactive power losses $=675.1011 \mathrm{kVAr}$

The minimum voltage value in p.u. $=0.885$

The minimum voltage is at bus 11 


\subsection{8-bus radial system}

This system is composed of 18 buses and 17 branches as shown in Figure 9. The bus data, line data and load flow solution of this system are given in Table 9 and they are driven from (Battu et al. 2016).

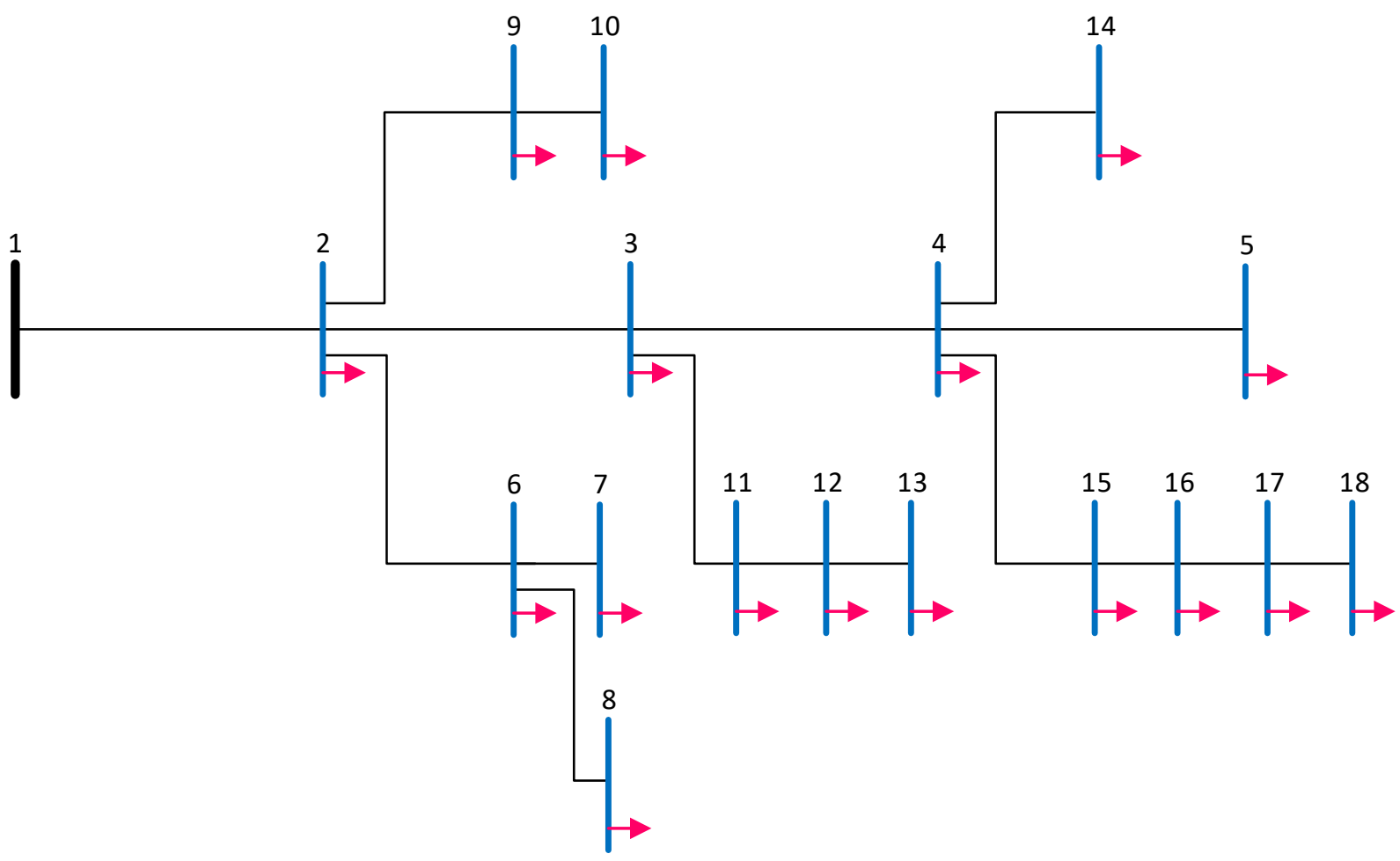

Figure 9 : Single line diagram of the 18-bus radial system.

Table 9 : Data and load flow solution for the 18-bus radial system.

\begin{tabular}{|c|c|c|c|c|c|c|c|c|}
\hline $\begin{array}{c}\text { Branch } \\
\mathbf{N}^{\circ}\end{array}$ & $\begin{array}{c}\text { Bus } \\
\text { From }\end{array}$ & $\begin{array}{c}\text { Bus } \\
\text { To }\end{array}$ & $\begin{array}{c}\mathbf{R} \\
\text { (p.u.) }\end{array}$ & $\begin{array}{c}\mathbf{X} \\
\text { (p.u.) }\end{array}$ & $\begin{array}{c}\text { PL } \\
\text { (Bus To) } \\
(\mathrm{kW})\end{array}$ & $\begin{array}{c}\text { QL } \\
\text { (Bus To) } \\
\text { (kVAR) }\end{array}$ & $\begin{array}{c}\mathrm{V} \\
\text { (Bus To) } \\
\text { (pu) } \\
\text { NR } \\
\end{array}$ & $\begin{array}{c}\mathrm{V} \\
\text { (Bus To) } \\
\text { (pu) } \\
\text { PQSUM } \\
\end{array}$ \\
\hline 1 & 1 & 2 & 0.7766 & 0.7596 & 44.1 & 45.0 & 0.9772 & 0.9772 \\
\hline 2 & 2 & 3 & 0.6716 & 0.6569 & 70.0 & 71.4 & 0.9646 & 0.9646 \\
\hline 3 & 3 & 4 & 0.4827 & 0.4722 & 140.0 & 142.8 & 0.9588 & 0.9588 \\
\hline 4 & 4 & 5 & 0.8744 & 0.5898 & 44.1 & 45.0 & 0.9552 & 0.9552 \\
\hline 5 & 2 & 9 & 1.1554 & 0.7793 & 70.0 & 71.4 & 0.9750 & 0.9750 \\
\hline 6 & 9 & 10 & 0.9680 & 0.6529 & 44.1 & 45.0 & 0.9742 & 0.9742 \\
\hline 7 & 2 & 6 & 1.4677 & 0.9900 & 140.0 & 142.8 & 0.9683 & 0.9683 \\
\hline 8 & 6 & 7 & 0.6245 & 0.4213 & 140.0 & 142.8 & 0.9668 & 0.9668 \\
\hline 9 & 6 & 8 & 0.7182 & 0.4844 & 70.0 & 71.4 & 0.9674 & 0.9674 \\
\hline 10 & 3 & 11 & 1.0305 & 0.6951 & 140.0 & 142.8 & 0.9600 & 0.9600 \\
\hline 11 & 11 & 12 & 1.4052 & 0.9478 & 70.0 & 71.4 & 0.9572 & 0.9572 \\
\hline 12 & 12 & 13 & 1.1554 & 0.7793 & 44.1 & 45.0 & 0.9563 & 0.9563 \\
\hline 13 & 4 & 14 & 1.2803 & 0.8636 & 70.0 & 71.4 & 0.9572 & 0.9572 \\
\hline 14 & 4 & 15 & 0.6870 & 0.4634 & 140.0 & 142.8 & 0.9571 & 0.9571 \\
\hline 15 & 5 & 16 & 0.6245 & 0.4213 & 70 & 71.4 & 0.9532 & 0.9532 \\
\hline 16 & 16 & 17 & 0.7182 & 0.4844 & 70 & 71.4 & 0.9517 & 0.9517 \\
\hline 17 & 17 & 18 & 0.7182 & 0.4844 & 44.1 & 45 & 0.9512 & 0.9512 \\
\hline
\end{tabular}


$\mathrm{S}_{\mathrm{base}}=100$ and $V_{\text {base }}=11 \mathrm{kV}$

The total active power load $=1410.5000 \mathrm{~kW}$

The total reactive power load $=1438.8000 \mathrm{kVAr}$

The total active power losses $=58.6080 \mathrm{~kW}$

The total reactive power losses $=54.6710 \mathrm{kVAr}$

The minimum voltage value in $\mathrm{p} . \mathrm{u} .=0.951$

The minimum voltage is at bus 18 


\subsection{2-bus radial system}

This system is composed of 22 buses and 21 branches as shown in Figure 10. The bus data, line data and load flow solution of this system are given in Table 10 and they are driven from (Ramalinga Raju et al. 2012).

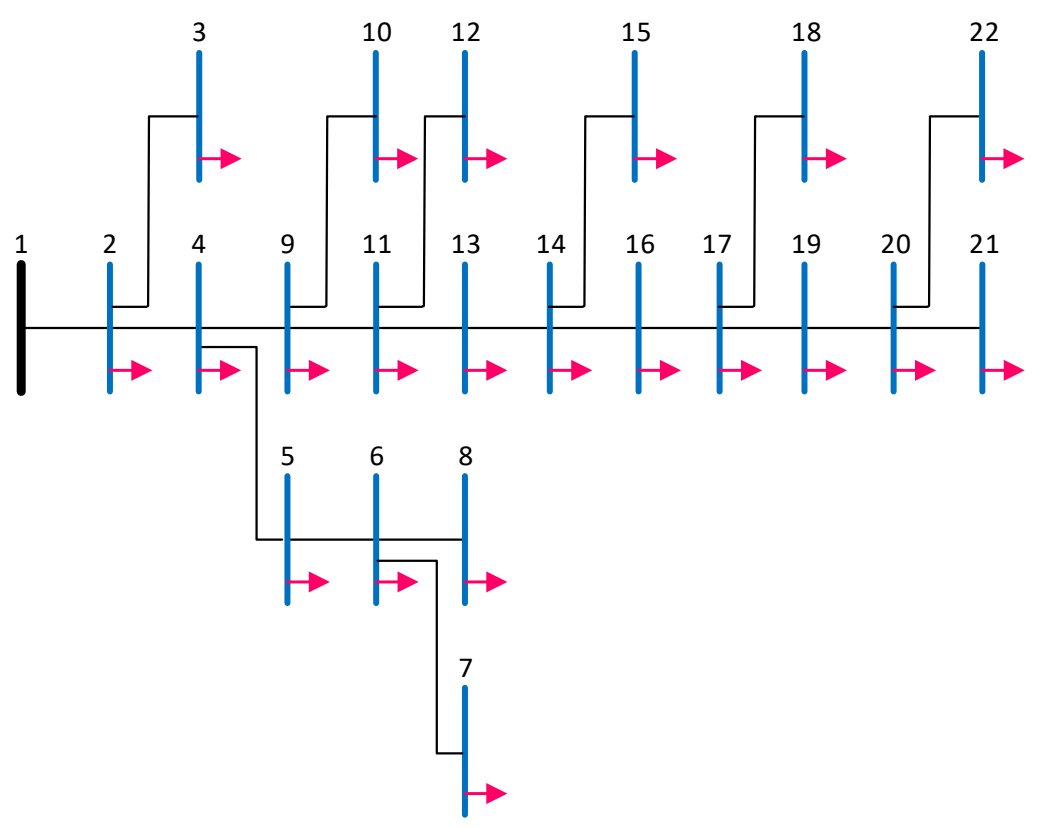

Figure 10 : Single line diagram of the 22-bus radial system.

Table 10 : Data and load flow solution for the 22-bus radial system.

\begin{tabular}{|c|c|c|c|c|c|c|c|c|}
\hline $\begin{array}{c}\text { Branch } \\
\mathrm{N}^{\circ}\end{array}$ & $\begin{array}{c}\text { Bus } \\
\text { From }\end{array}$ & $\begin{array}{c}\text { Bus } \\
\text { To }\end{array}$ & $\begin{array}{c}\mathbf{R} \\
\text { (p.u.) }\end{array}$ & $\begin{array}{c}\mathbf{X} \\
\text { (p.u.) }\end{array}$ & $\begin{array}{c}\text { PL } \\
\text { (Bus To) } \\
\text { (kW) }\end{array}$ & $\begin{array}{c}\text { QL } \\
\text { (Bus To) } \\
\text { (kVAR) }\end{array}$ & $\begin{array}{c}\mathbf{V} \\
\text { (Bus To) } \\
\text { (pu) } \\
\text { NR } \\
\end{array}$ & $\begin{array}{c}\mathrm{V} \\
\text { (Bus To) } \\
\text { (pu) } \\
\text { PQSUM } \\
\end{array}$ \\
\hline 1 & 1 & 2 & 0.3028 & 0.1493 & 16.8 & 20.9 & 0.9969 & 0.9969 \\
\hline 2 & 2 & 3 & 0.0452 & 0.0233 & 16.8 & 20.9 & 0.9969 & 0.9969 \\
\hline 3 & 2 & 4 & 0.4476 & 0.2305 & 33.8 & 37.3 & 0.9926 & 0.9926 \\
\hline 4 & 4 & 5 & 0.1595 & 0.0818 & 14.6 & 12.5 & 0.9925 & 0.9925 \\
\hline 5 & 4 & 9 & 0.6141 & 0.3163 & 14.4 & 18.6 & 0.9875 & 0.9875 \\
\hline 6 & 5 & 6 & 1.0835 & 0.5580 & 19.3 & 25.9 & 0.9918 & 0.9918 \\
\hline 7 & 6 & 7 & 0.0494 & 0.0255 & 10.5 & 14.2 & 0.9918 & 0.9918 \\
\hline 8 & 6 & 8 & 0.2401 & 0.1236 & 8.8 & 11.7 & 0.9917 & 0.9917 \\
\hline 9 & 9 & 10 & 0.0452 & 0.0233 & 14.4 & 18.6 & 0.9875 & 0.9875 \\
\hline 10 & 9 & 11 & 0.5579 & 0.2877 & 16.3 & 19.5 & 0.9832 & 0.9832 \\
\hline 11 & 11 & 12 & 0.0452 & 0.0233 & 16.3 & 19.5 & 0.9832 & 0.9832 \\
\hline 12 & 11 & 13 & 0.3258 & 0.1678 & 82.1 & 71.7 & 0.9808 & 0.9808 \\
\hline 13 & 13 & 14 & 0.8645 & 0.4453 & 34.7 & 30.1 & 0.9756 & 0.9756 \\
\hline 14 & 14 & 15 & 0.0182 & 0.0096 & 34.7 & 30.1 & 0.9756 & 0.9756 \\
\hline 15 & 14 & 16 & 0.0452 & 0.0233 & 80.3 & 70.1 & 0.9754 & 0.9754 \\
\hline 16 & 16 & 17 & 0.2655 & 0.1367 & 49.6 & 47.82 & 0.9744 & 0.9744 \\
\hline 17 & 17 & 18 & 0.0784 & 0.0403 & 49.6 & 47.82 & 0.9743 & 0.9743 \\
\hline 18 & 17 & 19 & 0.4744 & 0.2445 & 43.8 & 38.93 & 0.9733 & 0.9733 \\
\hline 19 & 19 & 20 & 0.1068 & 0.0545 & 37.32 & 35.96 & 0.9731 & 0.9731 \\
\hline 20 & 20 & 21 & 0.0720 & 0.0372 & 37.32 & 35.96 & 0.9731 & 0.9731 \\
\hline 21 & 20 & 22 & 0.4404 & 0.2268 & 31.02 & 29.36 & 0.9729 & 0.9729 \\
\hline
\end{tabular}


$\mathrm{S}_{\mathrm{base}}=100$ and $V_{\text {base }}=11 \mathrm{kV}$

Total active power load $=662.4600 \mathrm{~kW}$

Total reactive power load $=657.4500 \mathrm{kVAr}$

The total active power losses $=17.6732 \mathrm{~kW}$

The total reactive power losses $=9.0439 \mathrm{kVAr}$

The minimum voltage value in p.u. $=0.973$

The minimum voltage is at bus 22 


\subsection{8-bus radial system}

This system is composed of 28 buses and 27 branches as shown in Figure 9. The bus data, line data and load flow solution of this system are given in Table 9 and they are driven from (Das 1994).

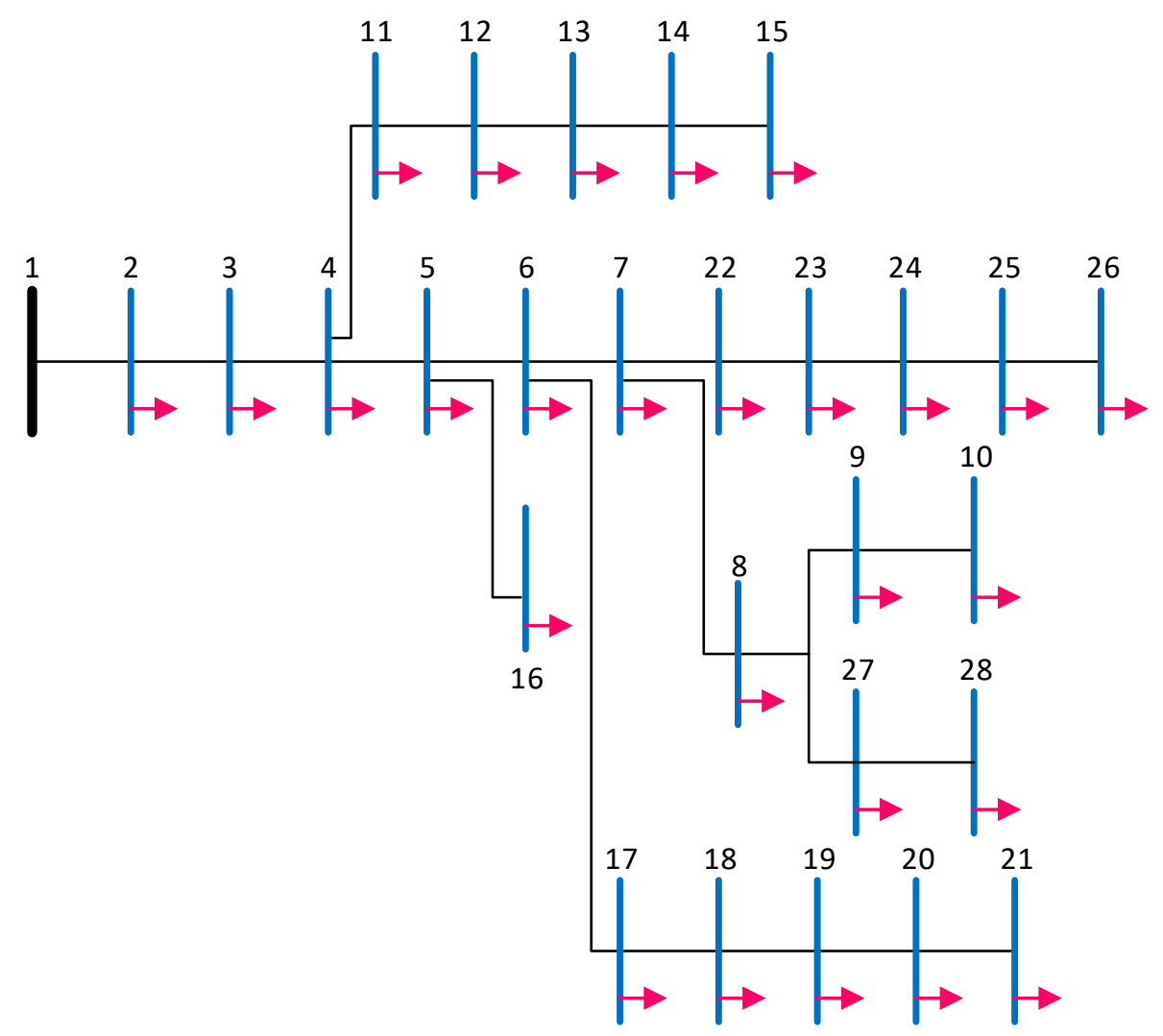

Figure 11 : Single line diagram of the 28-bus radial system. 
Table 11 : Data and load flow solution for the 28-bus radial system.

\begin{tabular}{|c|c|c|c|c|c|c|c|c|}
\hline $\begin{array}{c}\text { Branch } \\
\mathbf{N}^{\circ}\end{array}$ & $\begin{array}{c}\text { Bus } \\
\text { From }\end{array}$ & $\begin{array}{c}\text { Bus } \\
\text { To }\end{array}$ & $\begin{array}{c}\mathbf{R} \\
(\mathbf{p . u} .)\end{array}$ & $\begin{array}{c}\mathbf{X} \\
(\mathbf{p . u} .)\end{array}$ & $\begin{array}{c}\text { PL } \\
(\mathbf{B u s} \text { To) } \\
(\mathbf{k W})\end{array}$ & $\begin{array}{c}\mathbf{Q L} \\
(\mathbf{B u s} \text { To) } \\
(\mathbf{k V A R})\end{array}$ & $\begin{array}{c}\mathbf{V} \\
(\mathbf{B u s} \text { To) } \\
(\mathbf{p u}) \\
\mathbf{N R}\end{array}$ & $\begin{array}{c}\mathbf{V} \\
(\mathbf{B u s} \text { To) } \\
\mathbf{( p u )} \\
\mathbf{P Q S U M}\end{array}$ \\
\hline 1 & 1 & 2 & 0.9893 & 0.6777 & 35.280 & 35.993 & 0.9862 & 0.9862 \\
\hline 2 & 2 & 3 & 1.4843 & 1.0174 & 14.000 & 14.283 & 0.9665 & 0.9665 \\
\hline 3 & 3 & 4 & 1.0793 & 0.7397 & 35.280 & 35.993 & 0.9524 & 0.9524 \\
\hline 4 & 4 & 5 & 1.5298 & 1.0479 & 14.000 & 14.283 & 0.9382 & 0.9382 \\
\hline 5 & 5 & 6 & 1.2595 & 0.8628 & 35.280 & 35.993 & 0.9277 & 0.9277 \\
\hline 6 & 6 & 7 & 1.5744 & 1.0785 & 35.280 & 35.993 & 0.9185 & 0.9185 \\
\hline 7 & 7 & 8 & 0.9893 & 0.6777 & 35.280 & 35.993 & 0.9160 & 0.9160 \\
\hline 8 & 8 & 9 & 0.5397 & 0.3694 & 14.000 & 14.283 & 0.9158 & 0.9158 \\
\hline 9 & 9 & 10 & 0.9446 & 0.6471 & 14.000 & 14.283 & 0.9155 & 0.9155 \\
\hline 10 & 4 & 11 & 2.3331 & 0.9686 & 56.000 & 57.131 & 0.9462 & 0.9462 \\
\hline 11 & 11 & 12 & 0.9785 & 0.4058 & 35.280 & 35.993 & 0.9444 & 0.9444 \\
\hline 12 & 12 & 13 & 0.8281 & 0.3438 & 35.280 & 35.993 & 0.9433 & 0.9433 \\
\hline 13 & 13 & 14 & 0.3760 & 0.1562 & 14.000 & 14.283 & 0.9431 & 0.9431 \\
\hline 14 & 14 & 15 & 0.4512 & 0.1876 & 35.280 & 35.993 & 0.9428 & 0.9428 \\
\hline 15 & 5 & 16 & 2.1074 & 0.8744 & 35.280 & 35.993 & 0.9371 & 0.9371 \\
\hline 16 & 6 & 17 & 1.1289 & 0.4686 & 8.960 & 9.141 & 0.9259 & 0.9259 \\
\hline 17 & 17 & 18 & 0.6769 & 0.2810 & 8.960 & 9.141 & 0.9249 & 0.9249 \\
\hline 18 & 18 & 19 & 1.2793 & 0.5306 & 35.280 & 35.993 & 0.9232 & 0.9232 \\
\hline 19 & 19 & 20 & 1.1289 & 0.4686 & 35.280 & 35.993 & 0.9224 & 0.9224 \\
\hline 20 & 20 & 21 & 2.9355 & 1.2182 & 14.000 & 14.283 & 0.9217 & 0.9217 \\
\hline 21 & 7 & 22 & 1.2793 & 0.5306 & 35.280 & 35.993 & 0.9156 & 0.9156 \\
\hline 22 & 22 & 23 & 0.9025 & 0.3744 & 8.960 & 9.141 & 0.9141 & 0.9141 \\
\hline 23 & 23 & 24 & 0.7521 & 0.3124 & 56.000 & 57.131 & 0.9129 & 0.9129 \\
\hline 24 & 24 & 25 & 0.3760 & 0.1562 & 8.960 & 9.141 & 0.9126 & 0.9126 \\
\hline 25 & 25 & 26 & 0.3008 & 0.1248 & 35.280 & 35.993 & 0.9125 & 0.9125 \\
\hline 26 & 8 & 27 & 0.4512 & 0.1868 & 35.280 & 35.993 & 0.9155 & 0.9155 \\
\hline 27 & 27 & 28 & 0.2256 & 0.0934 & 35.280 & 35.993 & 0.9154 & 0.9154 \\
\hline
\end{tabular}

$\mathrm{S}_{\text {base }}=100$ and $V_{\text {base }}=11 \mathrm{kV}$

The total active power load $=761.0400 \mathrm{~kW}$

The total reactive power load $=776.4190 \mathrm{kVAr}$

The total active power losses $=68.8195 \mathrm{~kW}$

The total reactive power losses $=46.0420 \mathrm{kVAr}$

The minimum voltage value in p.u. $=0.912$

The minimum voltage is at bus 26 


\subsection{3-bus radial system}

This system is composed of 33 buses and 32 branches as shown in Figure 12. The bus data, line data and load flow solution of this system are given in Table 12 and they are driven from (Baran and Wu 1989a).

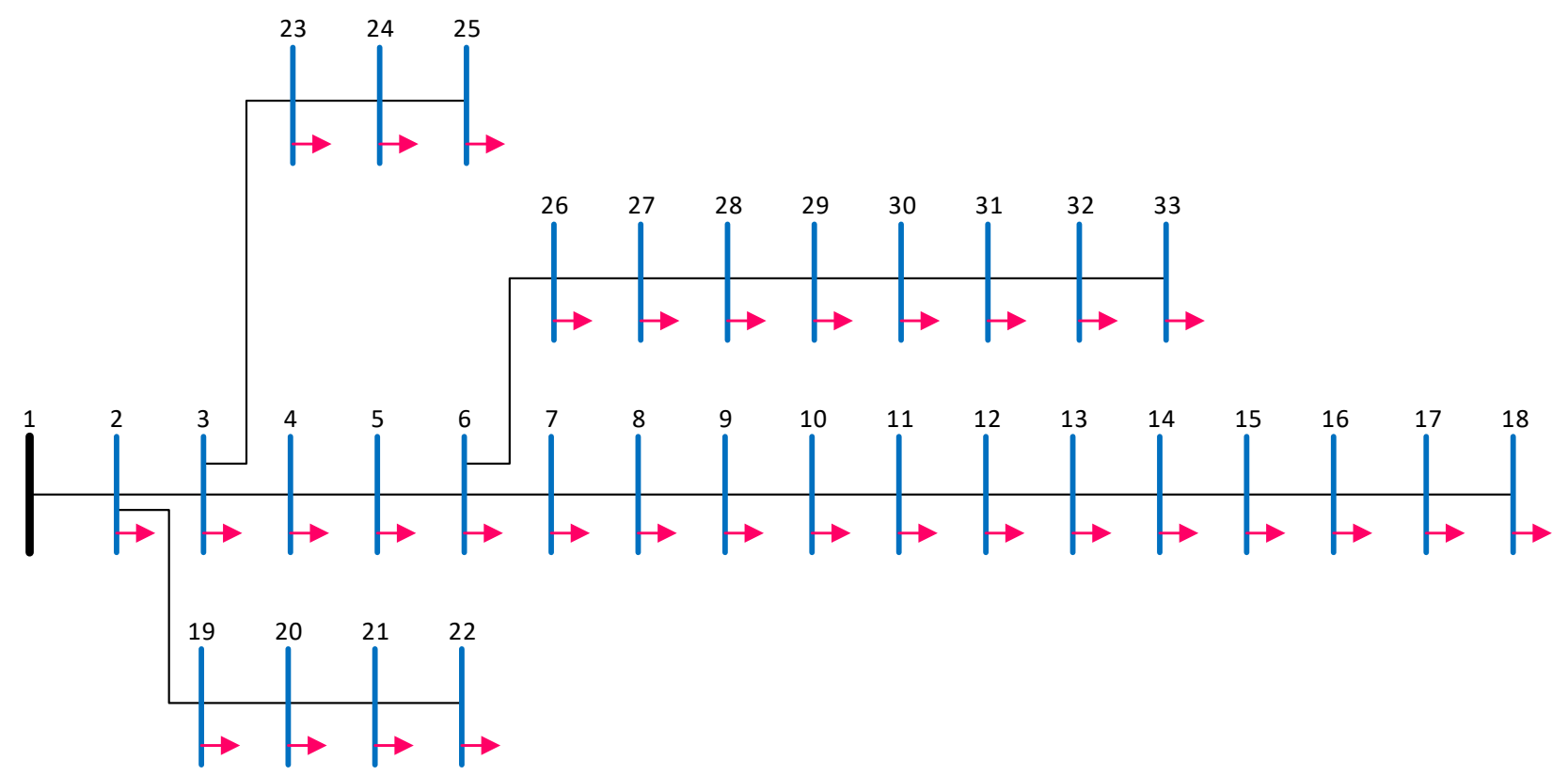

Figure 12 : Single line diagram of the 33-bus radial system. 
Table 12 : Data and load flow solution for the 33-bus radial system.

\begin{tabular}{|c|c|c|c|c|c|c|c|c|}
\hline $\begin{array}{c}\text { Branch } \\
\mathbf{N}^{\circ}\end{array}$ & $\begin{array}{c}\text { Bus } \\
\text { From }\end{array}$ & $\begin{array}{c}\text { Bus } \\
\text { To }\end{array}$ & $\begin{array}{c}\mathbf{R} \\
\text { (p.u.) }\end{array}$ & $\begin{array}{c}\mathbf{X} \\
\text { (p.u.) }\end{array}$ & $\begin{array}{c}\text { PL } \\
\text { (Bus To) } \\
\text { (kW) }\end{array}$ & $\begin{array}{c}\text { QL } \\
\text { (Bus To) } \\
\text { (kVAR) }\end{array}$ & $\begin{array}{c}\mathbf{V} \\
\text { (Bus To) } \\
\text { (pu) } \\
\text { NR }\end{array}$ & $\begin{array}{c}\mathbf{V} \\
\text { (Bus To) } \\
\text { (pu) } \\
\text { PQSUM }\end{array}$ \\
\hline 1 & 1 & 2 & 0.0575 & 0.0293 & 100.00 & 60.00 & 0.9970 & 0.9970 \\
\hline 2 & 2 & 3 & 0.3076 & 0.1567 & 90.00 & 40.00 & 0.9829 & 0.9829 \\
\hline 3 & 3 & 4 & 0.2284 & 0.1163 & 120.00 & 80.00 & 0.9755 & 0.9755 \\
\hline 4 & 4 & 5 & 0.2378 & 0.1211 & 60.00 & 30.00 & 0.9681 & 0.9681 \\
\hline 5 & 5 & 6 & 0.5110 & 0.4411 & 60.00 & 20.00 & 0.9497 & 0.9497 \\
\hline 6 & 6 & 7 & 0.1168 & 0.3861 & 200.00 & 100.00 & 0.9462 & 0.9462 \\
\hline 7 & 7 & 8 & 0.4439 & 0.1467 & 200.00 & 100.00 & 0.9413 & 0.9413 \\
\hline 8 & 8 & 9 & 0.6426 & 0.4617 & 60.00 & 20.00 & 0.9351 & 0.9351 \\
\hline 9 & 9 & 10 & 0.6514 & 0.4617 & 60.00 & 20.00 & 0.9292 & 0.9292 \\
\hline 10 & 10 & 11 & 0.1227 & 0.0406 & 45.00 & 30.00 & 0.9284 & 0.9284 \\
\hline 11 & 11 & 12 & 0.2336 & 0.0772 & 60.00 & 35.00 & 0.9269 & 0.9269 \\
\hline 12 & 12 & 13 & 0.9159 & 0.7206 & 60.00 & 35.00 & 0.9208 & 0.9208 \\
\hline 13 & 13 & 14 & 0.3379 & 0.4448 & 120.00 & 80.00 & 0.9185 & 0.9185 \\
\hline 14 & 14 & 15 & 0.3687 & 0.3282 & 60.00 & 10.00 & 0.9171 & 0.9171 \\
\hline 15 & 15 & 16 & 0.4656 & 0.3400 & 60.00 & 20.00 & 0.9157 & 0.9157 \\
\hline 16 & 16 & 17 & 0.8042 & 1.0738 & 60.00 & 20.00 & 0.9137 & 0.9137 \\
\hline 17 & 17 & 18 & 0.4567 & 0.3581 & 90.00 & 40.00 & 0.9131 & 0.9131 \\
\hline 18 & 2 & 19 & 0.1023 & 0.0976 & 90.00 & 40.00 & 0.9965 & 0.9965 \\
\hline 19 & 19 & 20 & 0.9385 & 0.8457 & 90.00 & 40.00 & 0.9929 & 0.9929 \\
\hline 20 & 20 & 21 & 0.2555 & 0.2985 & 90.00 & 40.00 & 0.9922 & 0.9922 \\
\hline 21 & 21 & 22 & 0.4423 & 0.5848 & 90.00 & 40.00 & 0.9916 & 0.9916 \\
\hline 22 & 3 & 23 & 0.2815 & 0.1924 & 90.00 & 50.00 & 0.9794 & 0.9794 \\
\hline 23 & 23 & 24 & 0.5603 & 0.4424 & 420.00 & 200.00 & 0.9727 & 0.9727 \\
\hline 24 & 24 & 25 & 0.5590 & 0.4374 & 420.00 & 200.00 & 0.9694 & 0.9694 \\
\hline 25 & 6 & 26 & 0.1267 & 0.0645 & 60.00 & 25.00 & 0.9477 & 0.9477 \\
\hline 26 & 26 & 27 & 0.1773 & 0.0903 & 60.00 & 25.00 & 0.9452 & 0.9452 \\
\hline 27 & 27 & 28 & 0.6607 & 0.5826 & 60.00 & 20.00 & 0.9337 & 0.9337 \\
\hline 28 & 28 & 29 & 0.5018 & 0.4371 & 120.00 & 70.00 & 0.9255 & 0.9255 \\
\hline 29 & 29 & 30 & 0.3166 & 0.1613 & 200.00 & 600.00 & 0.9220 & 0.9220 \\
\hline 30 & 30 & 31 & 0.6080 & 0.6008 & 150.00 & 70.00 & 0.9178 & 0.9178 \\
\hline 31 & 31 & 32 & 0.1937 & 0.2258 & 210.00 & 100.00 & 0.9169 & 0.9169 \\
\hline 32 & 32 & 33 & 0.2128 & 0.3308 & 60.00 & 40.00 & 0.9166 & 0.9166 \\
\hline
\end{tabular}

$S_{\text {base }}=100$ and $V_{\text {base }}=12.66 \mathrm{kV}$

The total active power load $=3715.0000 \mathrm{~kW}$

The total reactive power load $=2300.0000 \mathrm{kVAr}$

The total active power losses $=202.6771 \mathrm{~kW}$

The total reactive power losses $=135.1410 \mathrm{kVAr}$

The minimum voltage value in p.u. $=0.913$

The minimum voltage is at bus 18 


\subsection{4-bus radial system}

This system is composed of 34 buses and 33 branches as shown in Figure 13. The bus data, line data and load flow solution of this system are given in Table 13 and they are driven from (Salama and Chikhani 1993).

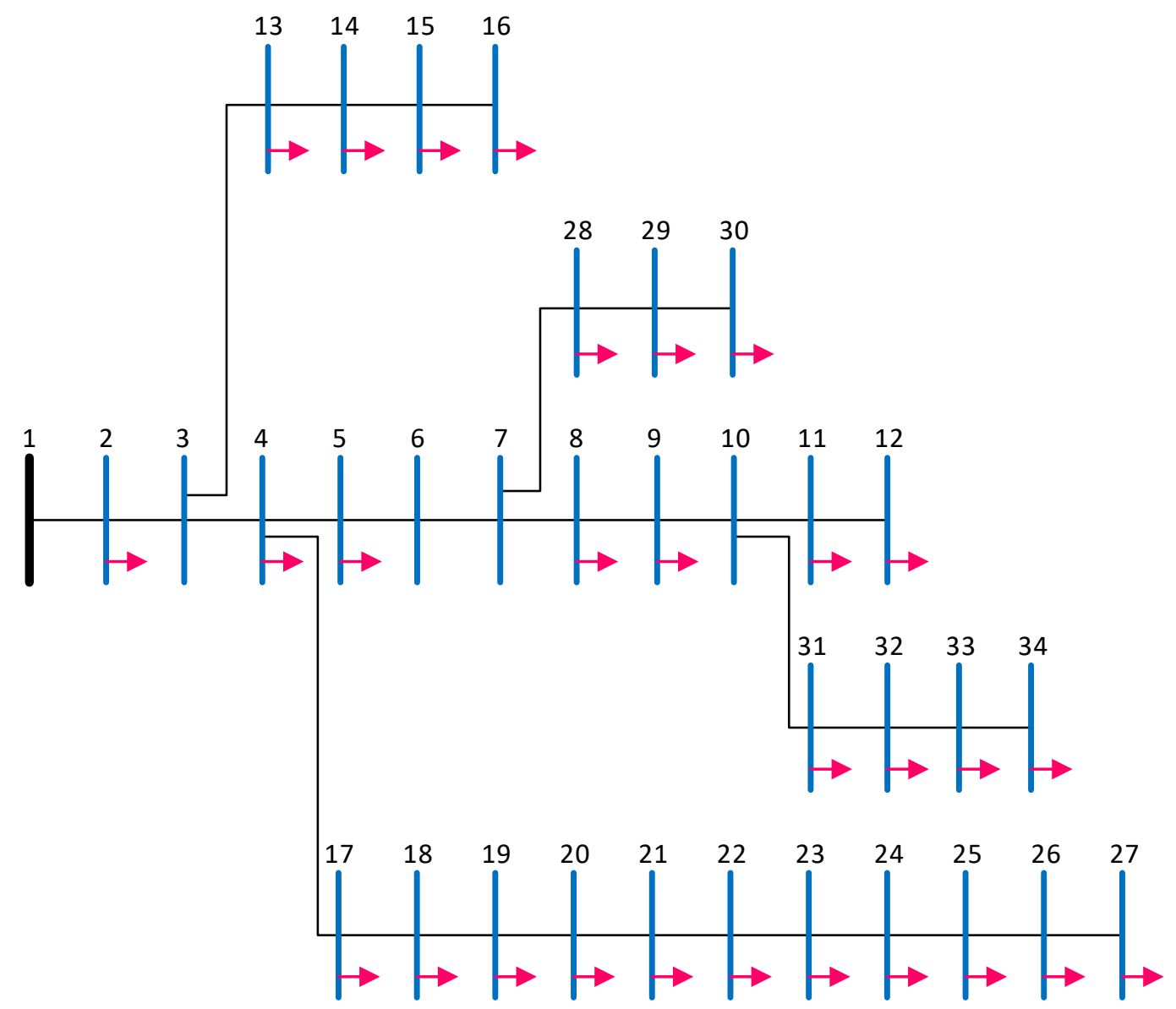

Figure 13 : Single line diagram of the 34-bus radial system. 
Table 13 : Data and load flow solution for the 34-bus radial system.

\begin{tabular}{|c|c|c|c|c|c|c|c|c|}
\hline $\begin{array}{c}\text { Branch } \\
\mathbf{N}^{\circ}\end{array}$ & $\begin{array}{c}\text { Bus } \\
\text { From }\end{array}$ & $\begin{array}{c}\text { Bus } \\
\text { To }\end{array}$ & $\begin{array}{c}\mathbf{R} \\
\text { (p.u.) }\end{array}$ & $\begin{array}{c}\mathbf{X} \\
\text { (p.u.) }\end{array}$ & $\begin{array}{c}\text { PL } \\
\text { (Bus To) } \\
(\mathbf{k W})\end{array}$ & $\begin{array}{c}\text { QL } \\
\text { (Bus To) } \\
\text { (kVAR) }\end{array}$ & $\begin{array}{c}\mathbf{V} \\
\text { (Bus To) } \\
\text { (pu) } \\
\text { NR } \\
\end{array}$ & $\begin{array}{c}\mathbf{V} \\
\text { (Bus To) } \\
\text { (pu) } \\
\text { PQSUM } \\
\end{array}$ \\
\hline 1 & 1 & 2 & 0.0967 & 0.0397 & 142.50 & 230.00 & 0.9952 & 0.9952 \\
\hline 2 & 2 & 3 & 0.0886 & 0.0364 & 0.00 & 0.00 & 0.9909 & 0.9909 \\
\hline 3 & 3 & 4 & 0.1359 & 0.0377 & 142.50 & 230.00 & 0.9856 & 0.9856 \\
\hline 4 & 4 & 5 & 0.1236 & 0.0343 & 142.50 & 230.00 & 0.9810 & 0.9810 \\
\hline 5 & 5 & 6 & 0.1236 & 0.0343 & 0.00 & 0.00 & 0.9766 & 0.9766 \\
\hline 6 & 6 & 7 & 0.2598 & 0.0446 & 0.00 & 0.00 & 0.9739 & 0.9739 \\
\hline 7 & 7 & 8 & 0.1732 & 0.0298 & 142.50 & 230.00 & 0.9724 & 0.9724 \\
\hline 8 & 8 & 9 & 0.2598 & 0.0446 & 142.50 & 230.00 & 0.9707 & 0.9707 \\
\hline 9 & 9 & 10 & 0.1732 & 0.0298 & 0.00 & 0.00 & 0.9698 & 0.9698 \\
\hline 10 & 10 & 11 & 0.1083 & 0.0186 & 142.50 & 230.00 & 0.9695 & 0.9695 \\
\hline 11 & 11 & 12 & 0.0866 & 0.0149 & 84.00 & 137.00 & 0.9694 & 0.9694 \\
\hline 12 & 3 & 13 & 0.1299 & 0.0223 & 45.00 & 72.00 & 0.9907 & 0.9907 \\
\hline 13 & 13 & 14 & 0.1732 & 0.0298 & 45.00 & 72.00 & 0.9905 & 0.9905 \\
\hline 14 & 14 & 15 & 0.0866 & 0.0149 & 45.00 & 72.00 & 0.9904 & 0.9904 \\
\hline 15 & 15 & 16 & 0.0433 & 0.0074 & 7.50 & 13.50 & 0.9904 & 0.9904 \\
\hline 16 & 6 & 17 & 0.1483 & 0.0412 & 142.50 & 230.00 & 0.9732 & 0.9732 \\
\hline 17 & 17 & 18 & 0.1359 & 0.0377 & 142.50 & 230.00 & 0.9704 & 0.9704 \\
\hline 18 & 18 & 19 & 0.1718 & 0.0391 & 142.50 & 230.00 & 0.9674 & 0.9674 \\
\hline 19 & 19 & 20 & 0.1562 & 0.0355 & 142.50 & 230.00 & 0.9649 & 0.9649 \\
\hline 20 & 20 & 21 & 0.1562 & 0.0355 & 142.50 & 230.00 & 0.9628 & 0.9628 \\
\hline 21 & 21 & 22 & 0.2165 & 0.0372 & 142.50 & 230.00 & 0.9605 & 0.9605 \\
\hline 22 & 22 & 23 & 0.2165 & 0.0372 & 142.50 & 230.00 & 0.9586 & 0.9586 \\
\hline 23 & 23 & 24 & 0.2598 & 0.0446 & 142.50 & 230.00 & 0.9568 & 0.9568 \\
\hline 24 & 24 & 25 & 0.1732 & 0.0298 & 142.50 & 230.00 & 0.9560 & 0.9560 \\
\hline 25 & 25 & 26 & 0.1083 & 0.0186 & 142.50 & 230.00 & 0.9556 & 0.9556 \\
\hline 26 & 26 & 27 & 0.0866 & 0.0149 & 85.00 & 137.00 & 0.9556 & 0.9556 \\
\hline 27 & 7 & 28 & 0.1299 & 0.0223 & 48.00 & 75.00 & 0.9737 & 0.9737 \\
\hline 28 & 28 & 29 & 0.1299 & 0.0223 & 48.00 & 75.00 & 0.9735 & 0.9735 \\
\hline 29 & 29 & 30 & 0.1299 & 0.0223 & 48.00 & 75.00 & 0.9734 & 0.9734 \\
\hline 30 & 10 & 31 & 0.1299 & 0.0223 & 34.50 & 57.00 & 0.9696 & 0.9696 \\
\hline 31 & 31 & 32 & 0.1732 & 0.0298 & 34.50 & 57.00 & 0.9694 & 0.9694 \\
\hline 32 & 32 & 33 & 0.1299 & 0.0223 & 34.50 & 57.00 & 0.9693 & 0.9693 \\
\hline 33 & 33 & 34 & 0.0866 & 0.0149 & 34.50 & 57.00 & 0.9692 & 0.9692 \\
\hline
\end{tabular}

$\mathrm{S}_{\text {base }}=100$ and $V_{\text {base }}=11 \mathrm{kV}$

The total active power load $=2873.5000 \mathrm{~kW}$

The total reactive power load $=4636.5000 \mathrm{kVAr}$

The total active power losses $=217.0102 \mathrm{~kW}$

The total reactive power losses $=63.7539 \mathrm{kVAr}$

The minimum voltage value in p.u. $=0.956$

The minimum voltage is at bus 27 


\subsection{8-bus radial system}

This system is composed of 38 buses and 37 branches as shown in Figure 14. The bus data, line data and load flow solution of this system are given in Table 14 and they are driven from (Singh and Misra 2007). It is worth to mention that this test system is similar to the 33-bus radial system except that the ending buses of tie lines are replaced by new busses that are buses $N^{\circ} 34,35,36,37$ and 38 .

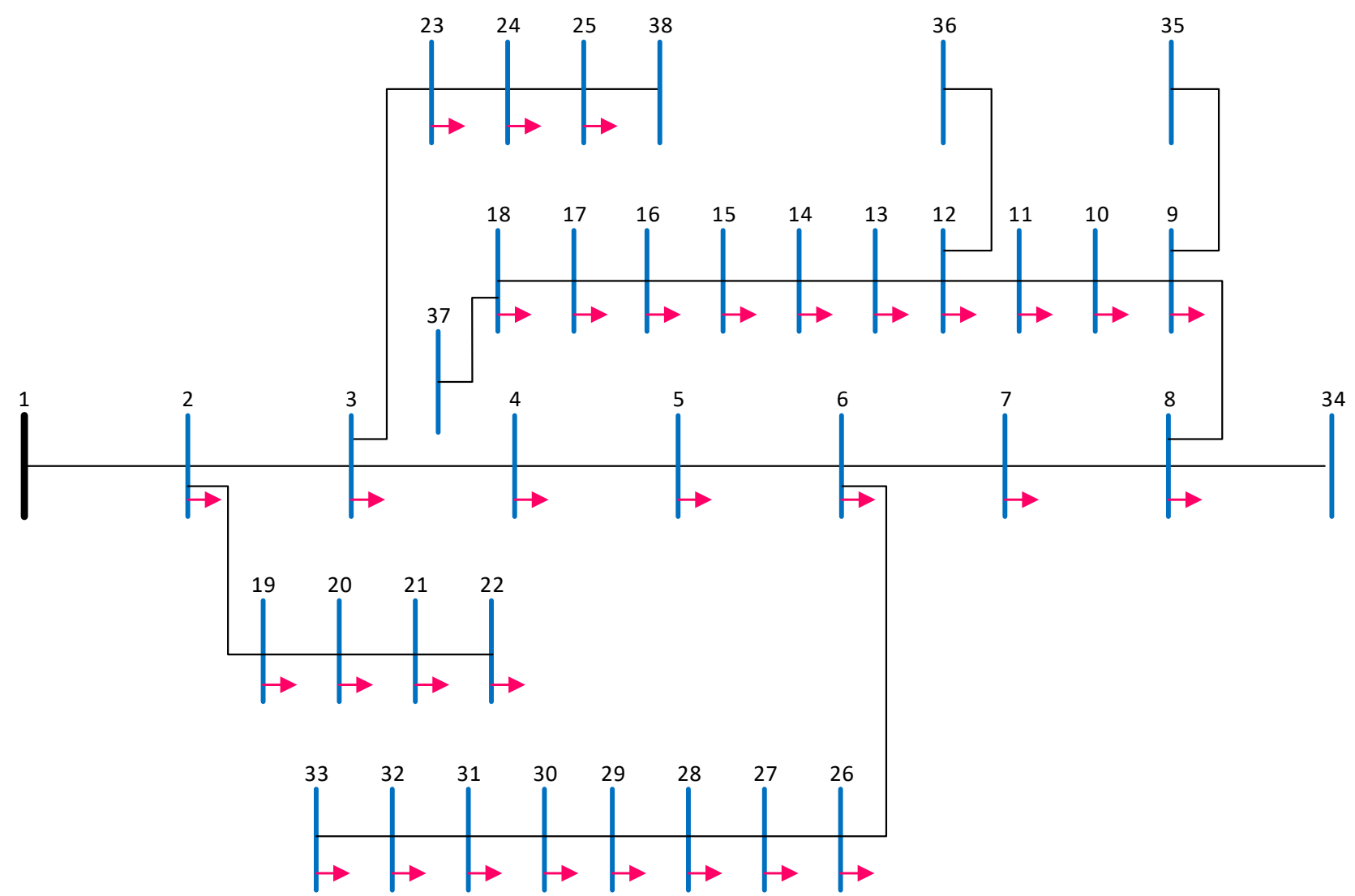

Figure 14 : Single line diagram of the 38-bus radial system. 
Table 14 : Data and load flow solution for the 38-bus radial system.

\begin{tabular}{|c|c|c|c|c|c|c|c|c|}
\hline $\begin{array}{c}\text { Branch } \\
\mathbf{N}^{\circ}\end{array}$ & $\begin{array}{c}\text { Bus } \\
\text { From }\end{array}$ & $\begin{array}{c}\text { Bus } \\
\text { To }\end{array}$ & $\begin{array}{c}\mathbf{R} \\
\text { (p.u.) }\end{array}$ & $\begin{array}{c}\mathbf{X} \\
\text { (p.u.) }\end{array}$ & $\begin{array}{c}\text { PL } \\
\text { (Bus To) } \\
\text { (kW) }\end{array}$ & $\begin{array}{c}\text { QL } \\
\text { (Bus To) } \\
\text { (kVAR) }\end{array}$ & $\begin{array}{c}\mathbf{V} \\
\text { (Bus To) } \\
\text { (pu) } \\
\text { NR } \\
\end{array}$ & $\begin{array}{c}\mathbf{V} \\
\text { (Bus To) } \\
\text { (pu) } \\
\text { PQSUM } \\
\end{array}$ \\
\hline 1 & 1 & 2 & 0.0575 & 0.0293 & 100.00 & 60.00 & 0.9970 & 0.9970 \\
\hline 2 & 2 & 3 & 0.3076 & 0.1567 & 90.00 & 40.00 & 0.9829 & 0.9829 \\
\hline 3 & 3 & 4 & 0.2284 & 0.1163 & 120.00 & 80.00 & 0.9755 & 0.9755 \\
\hline 4 & 4 & 5 & 0.2378 & 0.1211 & 60.00 & 30.00 & 0.9681 & 0.9681 \\
\hline 5 & 5 & 6 & 0.5110 & 0.4411 & 60.00 & 20.00 & 0.9497 & 0.9497 \\
\hline 6 & 6 & 7 & 0.1168 & 0.3861 & 200.00 & 100.00 & 0.9462 & 0.9462 \\
\hline 7 & 7 & 8 & 0.4439 & 0.1467 & 200.00 & 100.00 & 0.9413 & 0.9413 \\
\hline 8 & 8 & 9 & 0.6426 & 0.4617 & 60.00 & 20.00 & 0.9351 & 0.9351 \\
\hline 9 & 9 & 10 & 0.6514 & 0.4617 & 60.00 & 20.00 & 0.9292 & 0.9292 \\
\hline 10 & 10 & 11 & 0.1227 & 0.0406 & 45.00 & 30.00 & 0.9284 & 0.9284 \\
\hline 11 & 11 & 12 & 0.2336 & 0.0772 & 60.00 & 35.00 & 0.9269 & 0.9269 \\
\hline 12 & 12 & 13 & 0.9159 & 0.7206 & 60.00 & 35.00 & 0.9208 & 0.9208 \\
\hline 13 & 13 & 14 & 0.3379 & 0.4448 & 120.00 & 80.00 & 0.9185 & 0.9185 \\
\hline 14 & 14 & 15 & 0.3687 & 0.3282 & 60.00 & 10.00 & 0.9171 & 0.9171 \\
\hline 15 & 15 & 16 & 0.4656 & 0.3400 & 60.00 & 20.00 & 0.9157 & 0.9157 \\
\hline 16 & 16 & 17 & 0.8042 & 1.0738 & 60.00 & 20.00 & 0.9137 & 0.9137 \\
\hline 17 & 17 & 18 & 0.4567 & 0.3581 & 90.00 & 40.00 & 0.9131 & 0.9131 \\
\hline 18 & 2 & 19 & 0.1023 & 0.0976 & 90.00 & 40.00 & 0.9965 & 0.9965 \\
\hline 19 & 19 & 20 & 0.9385 & 0.8457 & 90.00 & 40.00 & 0.9929 & 0.9929 \\
\hline 20 & 20 & 21 & 0.2555 & 0.2985 & 90.00 & 40.00 & 0.9922 & 0.9922 \\
\hline 21 & 21 & 22 & 0.4423 & 0.5848 & 90.00 & 40.00 & 0.9916 & 0.9916 \\
\hline 22 & 3 & 23 & 0.2815 & 0.1924 & 90.00 & 50.00 & 0.9794 & 0.9794 \\
\hline 23 & 23 & 24 & 0.5603 & 0.4424 & 420.00 & 200.00 & 0.9727 & 0.9727 \\
\hline 24 & 24 & 25 & 0.5590 & 0.4374 & 420.00 & 200.00 & 0.9694 & 0.9694 \\
\hline 25 & 6 & 26 & 0.1267 & 0.0645 & 60.00 & 25.00 & 0.9477 & 0.9477 \\
\hline 26 & 26 & 27 & 0.1773 & 0.0903 & 60.00 & 25.00 & 0.9452 & 0.9452 \\
\hline 27 & 27 & 28 & 0.6607 & 0.5826 & 60.00 & 20.00 & 0.9337 & 0.9337 \\
\hline 28 & 28 & 29 & 0.5018 & 0.4371 & 120.00 & 70.00 & 0.9255 & 0.9255 \\
\hline 29 & 29 & 30 & 0.3166 & 0.1613 & 200.00 & 600.00 & 0.9220 & 0.9220 \\
\hline 30 & 30 & 31 & 0.6080 & 0.6008 & 150.00 & 70.00 & 0.9178 & 0.9178 \\
\hline 31 & 31 & 32 & 0.1937 & 0.2258 & 210.00 & 100.00 & 0.9169 & 0.9169 \\
\hline 32 & 32 & 33 & 0.2128 & 0.3308 & 60.00 & 40.00 & 0.9166 & 0.9166 \\
\hline 33 & 8 & 34 & 1.2479 & 1.2479 & 0.00 & 0.00 & 0.9413 & 0.9413 \\
\hline 34 & 9 & 35 & 1.2479 & 1.2479 & 0.00 & 0.00 & 0.9351 & 0.9351 \\
\hline 35 & 12 & 36 & 1.2479 & 1.2479 & 0.00 & 0.00 & 0.9269 & 0.9269 \\
\hline 36 & 18 & 37 & 0.3120 & 0.3120 & 0.00 & 0.00 & 0.9131 & 0.9131 \\
\hline 37 & 25 & 38 & 0.3120 & 0.3120 & 0.00 & 0.00 & 0.9694 & 0.9694 \\
\hline
\end{tabular}

$\mathrm{S}_{\text {base }}=100$ and $V_{\text {base }}=12.66 \mathrm{kV}$

The total active power load $=3715.0000 \mathrm{~kW}$

The total reactive power load $=2300.0000 \mathrm{kVAr}$

The total active power losses $=202.6771 \mathrm{~kW}$

The total reactive power losses $=135.1410 \mathrm{kVAr}$

The minimum voltage value in p.u. $=0.913$

The minimum voltage is at bus 18 


\subsection{1-bus radial system}

There are two 51-bus radial test system systems that we have found in the literature.

\subsubsection{System 1}

This system is composed of 51 buses and 50 branches as shown in Figure 15. The bus data, line data and load flow solution of this system are given in Table 15 and they are driven from (Gampa and Das 2015).

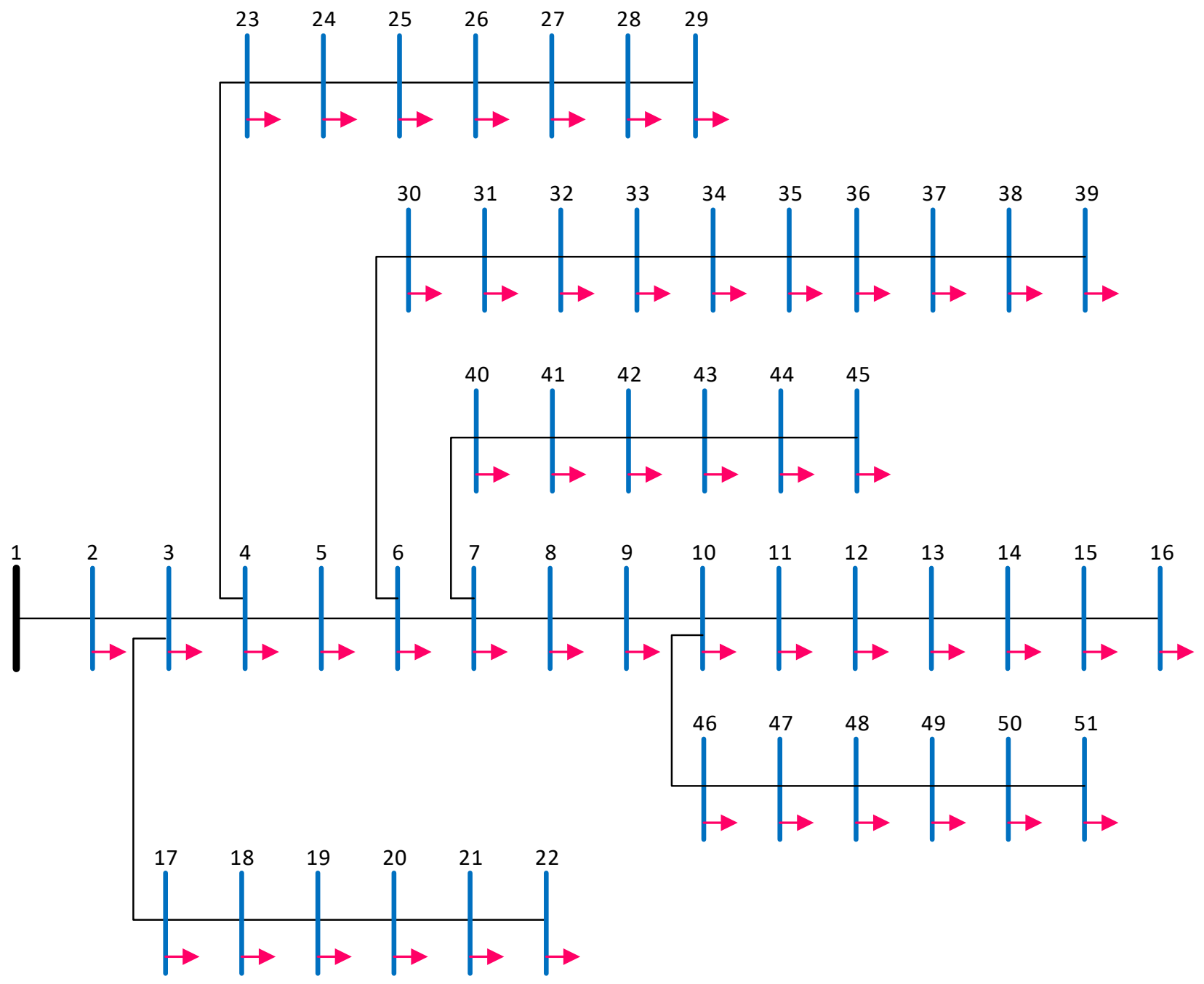

Figure 15 : Single line diagram of the 51-bus radial system. 
Table 15 : Data and load flow solution for the 51-bus radial system.

\begin{tabular}{|c|c|c|c|c|c|c|c|c|}
\hline $\begin{array}{c}\text { Branch } \\
\mathbf{N}^{\circ}\end{array}$ & $\begin{array}{c}\text { Bus } \\
\text { From }\end{array}$ & $\begin{array}{c}\text { Bus } \\
\text { To }\end{array}$ & $\begin{array}{c}\mathbf{R} \\
\text { (p.u.) }\end{array}$ & $\begin{array}{c}\mathbf{X} \\
\text { (p.u.) }\end{array}$ & $\begin{array}{c}\text { PL } \\
\text { (Bus To) } \\
(\mathbf{k W})\end{array}$ & $\begin{array}{c}\text { QL } \\
\text { (Bus To) } \\
\text { (kVAR) }\end{array}$ & $\begin{array}{c}\mathbf{V} \\
\text { (Bus To) } \\
\text { (pu) } \\
\text { NR } \\
\end{array}$ & $\begin{array}{c}\mathbf{V} \\
\text { (Bus To) } \\
\text { (pu) } \\
\text { PQSUM } \\
\end{array}$ \\
\hline 1 & 1 & 2 & 0.2264 & 0.2942 & 40 & 30 & 0.9892 & 0.9892 \\
\hline 2 & 2 & 3 & 0.1132 & 0.1471 & 60 & 40 & 0.9839 & 0.9839 \\
\hline 3 & 3 & 4 & 0.2717 & 0.3531 & 20 & 10 & 0.9731 & 0.9731 \\
\hline 4 & 4 & 5 & 0.0906 & 0.1177 & 80 & 60 & 0.9701 & 0.9701 \\
\hline 5 & 5 & 6 & 0.4463 & 0.3600 & 38 & 18 & 0.9584 & 0.9584 \\
\hline 6 & 6 & 7 & 0.2975 & 0.2400 & 20 & 15 & 0.9526 & 0.9526 \\
\hline 7 & 7 & 8 & 0.2975 & 0.2400 & 60 & 40 & 0.9487 & 0.9487 \\
\hline 8 & 8 & 9 & 0.5950 & 0.4800 & 70 & 45 & 0.9413 & 0.9413 \\
\hline 9 & 9 & 10 & 2.2579 & 0.6440 & 60 & 35 & 0.9299 & 0.9299 \\
\hline 10 & 10 & 11 & 1.6934 & 0.4830 & 80 & 50 & 0.9226 & 0.9226 \\
\hline 11 & 11 & 12 & 1.6934 & 0.4830 & 10 & 5 & 0.9171 & 0.9171 \\
\hline 12 & 12 & 13 & 0.7902 & 0.2254 & 25 & 15 & 0.9146 & 0.9146 \\
\hline 13 & 13 & 14 & 0.9031 & 0.2576 & 55 & 45 & 0.9120 & 0.9120 \\
\hline 14 & 14 & 15 & 1.2418 & 0.3542 & 120 & 80 & 0.9094 & 0.9094 \\
\hline 15 & 15 & 16 & 2.4836 & 0.7083 & 40 & 25 & 0.9081 & 0.9081 \\
\hline 16 & 3 & 17 & 2.2579 & 0.6440 & 35 & 25 & 0.9747 & 0.9747 \\
\hline 17 & 17 & 18 & 0.6774 & 0.1932 & 60 & 30 & 0.9723 & 0.9723 \\
\hline 18 & 18 & 19 & 1.1289 & 0.3220 & 80 & 50 & 0.9690 & 0.9690 \\
\hline 19 & 19 & 20 & 1.1289 & 0.3220 & 60 & 35 & 0.9668 & 0.9668 \\
\hline 20 & 20 & 21 & 1.6934 & 0.4830 & 50 & 30 & 0.9648 & 0.9648 \\
\hline 21 & 21 & 22 & 1.2418 & 0.3542 & 50 & 30 & 0.9640 & 0.9640 \\
\hline 22 & 4 & 23 & 1.3547 & 0.3864 & 80 & 60 & 0.9679 & 0.9679 \\
\hline 23 & 23 & 24 & 1.4676 & 0.4186 & 45 & 25 & 0.9637 & 0.9637 \\
\hline 24 & 24 & 25 & 0.9031 & 0.2576 & 38 & 18 & 0.9616 & 0.9616 \\
\hline 25 & 25 & 26 & 0.6774 & 0.1932 & 78 & 48 & 0.9603 & 0.9603 \\
\hline 26 & 26 & 27 & 0.4516 & 0.1288 & 16 & 8 & 0.9599 & 0.9599 \\
\hline 27 & 27 & 28 & 0.9031 & 0.2576 & 18 & 10 & 0.9593 & 0.9593 \\
\hline 28 & 28 & 29 & 0.2258 & 0.0644 & 40 & 30 & 0.9592 & 0.9592 \\
\hline 29 & 6 & 30 & 0.5802 & 0.3945 & 40 & 30 & 0.9551 & 0.9551 \\
\hline 30 & 30 & 31 & 0.5355 & 0.3641 & 20 & 15 & 0.9524 & 0.9524 \\
\hline 31 & 31 & 32 & 0.5355 & 0.3641 & 30 & 20 & 0.9498 & 0.9498 \\
\hline 32 & 32 & 33 & 0.5355 & 0.3641 & 36 & 26 & 0.9475 & 0.9475 \\
\hline 33 & 33 & 34 & 0.4463 & 0.3035 & 50 & 40 & 0.9458 & 0.9458 \\
\hline 34 & 34 & 35 & 0.2678 & 0.1821 & 27 & 18 & 0.9450 & 0.9450 \\
\hline 35 & 35 & 36 & 0.3213 & 0.2185 & 33 & 16 & 0.9442 & 0.9442 \\
\hline 36 & 36 & 37 & 0.3570 & 0.2428 & 42 & 22 & 0.9435 & 0.9435 \\
\hline 37 & 37 & 38 & 0.4909 & 0.3338 & 55 & 30 & 0.9428 & 0.9428 \\
\hline 38 & 38 & 39 & 0.5802 & 0.3945 & 44 & 26 & 0.9424 & 0.9424 \\
\hline 39 & 7 & 40 & 1.5805 & 0.4507 & 80 & 70 & 0.9451 & 0.9451 \\
\hline 40 & 40 & 41 & 2.4836 & 0.7083 & 60 & 30 & 0.9360 & 0.9360 \\
\hline 41 & 41 & 42 & 2.0321 & 0.5796 & 45 & 30 & 0.9300 & 0.9300 \\
\hline 42 & 42 & 43 & 1.8063 & 0.5152 & 48 & 28 & 0.9257 & 0.9257 \\
\hline 43 & 43 & 44 & 1.8063 & 0.5152 & 68 & 38 & 0.9226 & 0.9226 \\
\hline 44 & 44 & 45 & 0.5645 & 0.1610 & 77 & 23 & 0.9221 & 0.9221 \\
\hline 45 & 9 & 46 & 0.7902 & 0.2254 & 60 & 30 & 0.9385 & 0.9385 \\
\hline 46 & 46 & 47 & 0.8467 & 0.2415 & 40 & 20 & 0.9361 & 0.9361 \\
\hline 47 & 47 & 48 & 1.0160 & 0.2898 & 45 & 45 & 0.9336 & 0.9336 \\
\hline 48 & 48 & 49 & 1.4676 & 0.4186 & 70 & 50 & 0.9311 & 0.9311 \\
\hline 49 & 49 & 50 & 1.3547 & 0.3864 & 30 & 20 & 0.9299 & 0.9299 \\
\hline 50 & 50 & 51 & 1.1289 & 0.3220 & 35 & 30 & 0.9294 & 0.9294 \\
\hline
\end{tabular}


$\mathrm{S}_{\text {base }}=100$ and $V_{\text {base }}=11 \mathrm{kV}$

The total active power load $=2463.0000 \mathrm{~kW}$

The total reactive power load $=1569.0000 \mathrm{kVAr}$

The total active power losses $=129.5559 \mathrm{~kW}$

The total reactive power losses $=111.6835 \mathrm{kVAr}$

The minimum voltage value in p.u. $=0.908$

The minimum voltage is at bus 16

\subsubsection{System 2}

This system is composed of 51 buses and 50 branches as shown in Figure 16. The bus data, line data and load flow solution of this system are given in Table 16 and they are driven from (Hengsritawat et al. 2012).

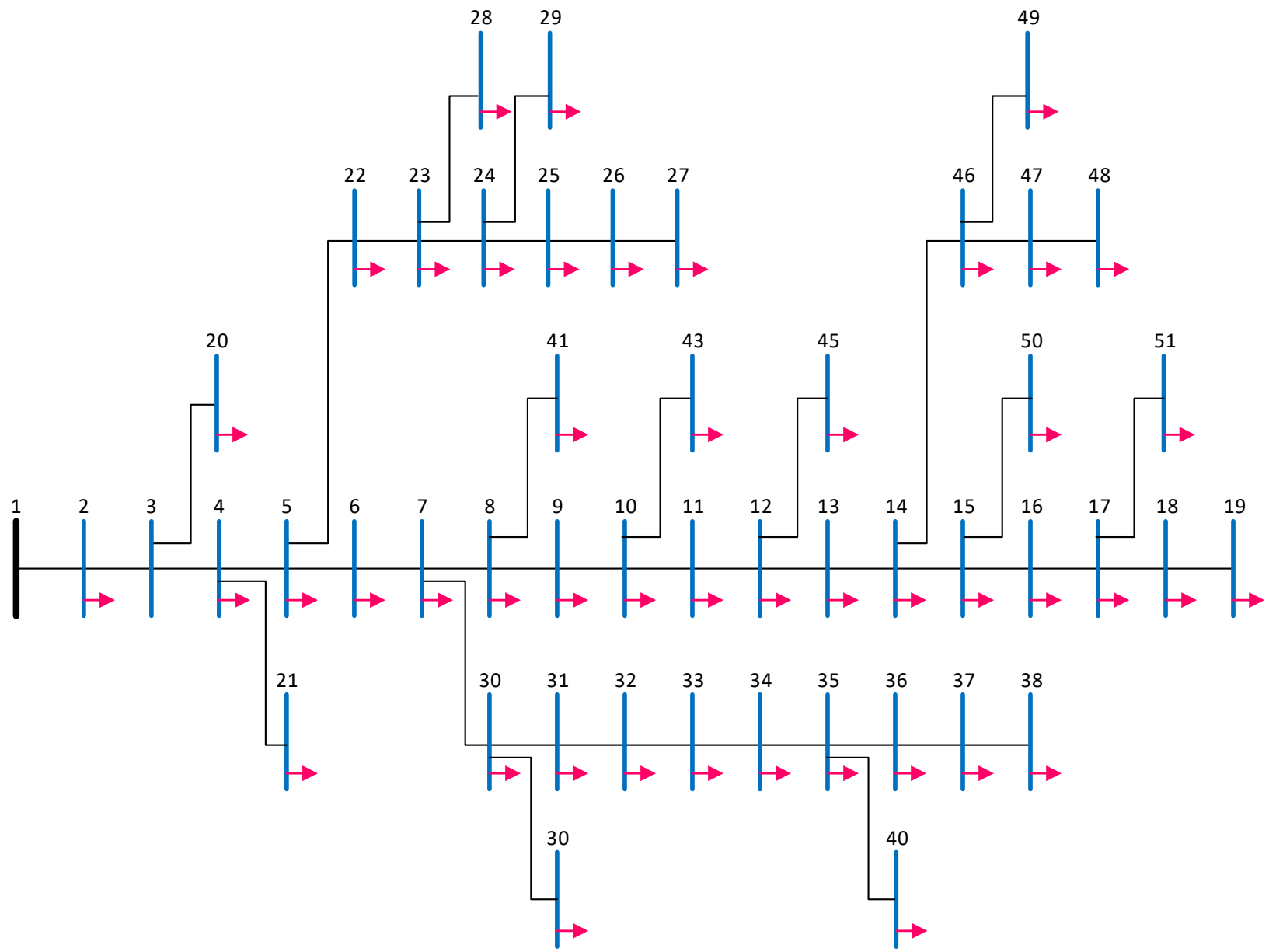

Figure 16 : Single line diagram of the 51-bus radial system. 
Table 16 : Data and load flow solution for the 51-bus radial system.

\begin{tabular}{|c|c|c|c|c|c|c|c|c|}
\hline $\begin{array}{c}\text { Branch } \\
\mathbf{N}^{\circ}\end{array}$ & $\begin{array}{c}\text { Bus } \\
\text { From }\end{array}$ & $\begin{array}{c}\text { Bus } \\
\text { To }\end{array}$ & $\begin{array}{c}\mathbf{R} \\
\text { (p.u.) }\end{array}$ & $\begin{array}{c}\mathbf{X} \\
\text { (p.u.) }\end{array}$ & $\begin{array}{c}\text { PL } \\
\text { (Bus To) } \\
\text { (kW) }\end{array}$ & $\begin{array}{c}\text { QL } \\
\text { (Bus To) } \\
\text { (kVAR) }\end{array}$ & $\begin{array}{c}\mathbf{V} \\
\text { (Bus To) } \\
\text { (pu) } \\
\text { NR } \\
\end{array}$ & $\begin{array}{c}\mathbf{V} \\
\text { (Bus To) } \\
\text { (pu) } \\
\text { PQSUM } \\
\end{array}$ \\
\hline 1 & 1 & 2 & 0.0871 & 0.1515 & 14.58 & 8.07 & 0.9966 & 0.9966 \\
\hline 2 & 2 & 3 & 0.0871 & 0.1515 & 0.00 & 0.00 & 0.9933 & 0.9933 \\
\hline 3 & 3 & 4 & 0.0435 & 0.0758 & 0.00 & 0.00 & 0.9916 & 0.9916 \\
\hline 4 & 4 & 5 & 0.0871 & 0.1515 & 0.00 & 0.00 & 0.9884 & 0.9884 \\
\hline 5 & 5 & 6 & 0.0435 & 0.0758 & 14.58 & 8.07 & 0.9871 & 0.9871 \\
\hline 6 & 6 & 7 & 0.0435 & 0.0758 & 0.00 & 0.00 & 0.9858 & 0.9858 \\
\hline 7 & 7 & 8 & 0.0871 & 0.1515 & 0.00 & 0.00 & 0.9841 & 0.9841 \\
\hline 8 & 8 & 9 & 0.0871 & 0.1515 & 58.33 & 32.27 & 0.9824 & 0.9824 \\
\hline 9 & 9 & 10 & 0.0826 & 0.1389 & 0.00 & 0.00 & 0.9811 & 0.9811 \\
\hline 10 & 10 & 11 & 0.1101 & 0.1852 & 0.00 & 0.00 & 0.9793 & 0.9793 \\
\hline 11 & 11 & 12 & 0.0550 & 0.0926 & 20.00 & 15.00 & 0.9786 & 0.9786 \\
\hline 12 & 12 & 13 & 0.1651 & 0.2777 & 0.00 & 0.00 & 0.9766 & 0.9766 \\
\hline 13 & 13 & 14 & 0.1101 & 0.1852 & 0.00 & 0.00 & 0.9752 & 0.9752 \\
\hline 14 & 14 & 15 & 0.3444 & 0.2277 & 0.00 & 0.00 & 0.9729 & 0.9729 \\
\hline 15 & 15 & 16 & 0.4132 & 0.2732 & 29.17 & 16.14 & 0.9708 & 0.9708 \\
\hline 16 & 16 & 17 & 0.1377 & 0.0911 & 72.92 & 40.34 & 0.9701 & 0.9701 \\
\hline 17 & 17 & 18 & 0.2755 & 0.1821 & 20.00 & 15.00 & 0.9695 & 0.9695 \\
\hline 18 & 18 & 19 & 0.1377 & 0.0911 & 145.67 & 80.69 & 0.9692 & 0.9692 \\
\hline 19 & 3 & 20 & 1.1020 & 0.7286 & 62.88 & 33.33 & 0.9923 & 0.9923 \\
\hline 20 & 4 & 21 & 0.2755 & 0.1821 & 14.58 & 8.07 & 0.9916 & 0.9916 \\
\hline 21 & 5 & 22 & 0.6887 & 0.4554 & 94.79 & 52.45 & 0.9855 & 0.9855 \\
\hline 22 & 22 & 23 & 0.5510 & 0.3643 & 14.58 & 8.07 & 0.9838 & 0.9838 \\
\hline 23 & 23 & 24 & 0.1377 & 0.0911 & 0.00 & 0.00 & 0.9835 & 0.9835 \\
\hline 24 & 24 & 25 & 1.3775 & 0.9107 & 14.58 & 8.07 & 0.9820 & 0.9820 \\
\hline 25 & 25 & 26 & 0.2755 & 0.1821 & 35.00 & 19.36 & 0.9817 & 0.9817 \\
\hline 26 & 26 & 27 & 0.4132 & 0.2732 & 29.17 & 16.14 & 0.9816 & 0.9816 \\
\hline 27 & 23 & 28 & 1.1020 & 0.7286 & 29.17 & 16.14 & 0.9834 & 0.9834 \\
\hline 28 & 24 & 29 & 0.2755 & 0.1821 & 91.88 & 50.83 & 0.9832 & 0.9832 \\
\hline 29 & 7 & 30 & 1.0331 & 0.6831 & 85.90 & 36.83 & 0.9778 & 0.9778 \\
\hline 30 & 30 & 31 & 0.1377 & 0.0911 & 29.17 & 16.14 & 0.9770 & 0.9770 \\
\hline 31 & 31 & 32 & 0.2755 & 0.1821 & 14.58 & 8.07 & 0.9755 & 0.9755 \\
\hline 32 & 32 & 33 & 0.2755 & 0.1821 & 43.75 & 24.21 & 0.9740 & 0.9740 \\
\hline 33 & 33 & 34 & 0.2066 & 0.1366 & 43.75 & 24.21 & 0.9731 & 0.9731 \\
\hline 34 & 34 & 35 & 0.2755 & 0.1821 & 0.00 & 0.00 & 0.9720 & 0.9720 \\
\hline 35 & 35 & 36 & 0.4821 & 0.3188 & 145.83 & 80.69 & 0.9702 & 0.9702 \\
\hline 36 & 36 & 37 & 0.2755 & 0.1821 & 91.88 & 50.83 & 0.9697 & 0.9697 \\
\hline 37 & 37 & 38 & 0.2066 & 0.1366 & 29.17 & 16.14 & 0.9696 & 0.9696 \\
\hline 38 & 30 & 39 & 0.4132 & 0.2732 & 58.33 & 32.27 & 0.9774 & 0.9774 \\
\hline 39 & 35 & 40 & 0.2755 & 0.1821 & 14.58 & 8.07 & 0.9720 & 0.9720 \\
\hline 40 & 8 & 41 & 0.2066 & 0.1366 & 29.17 & 16.14 & 0.9840 & 0.9840 \\
\hline 41 & 9 & 42 & 0.2755 & 0.1821 & 29.17 & 16.14 & 0.9823 & 0.9823 \\
\hline 42 & 10 & 43 & 0.8265 & 0.5464 & 29.17 & 16.14 & 0.9807 & 0.9807 \\
\hline 43 & 11 & 44 & 0.2755 & 0.1821 & 148.75 & 82.30 & 0.9787 & 0.9787 \\
\hline 44 & 12 & 45 & 0.9642 & 0.6375 & 29.17 & 16.14 & 0.9782 & 0.9782 \\
\hline 45 & 14 & 46 & 0.1377 & 0.0911 & 0.00 & 0.00 & 0.9750 & 0.9750 \\
\hline 46 & 46 & 47 & 0.4132 & 0.2732 & 29.17 & 16.14 & 0.9745 & 0.9745 \\
\hline 47 & 47 & 48 & 0.4132 & 0.2732 & 58.33 & 32.27 & 0.9741 & 0.9741 \\
\hline 48 & 46 & 49 & 0.0275 & 0.0182 & 43.75 & 24.21 & 0.9749 & 0.9749 \\
\hline 49 & 15 & 50 & 0.9642 & 0.6375 & 116.67 & 64.55 & 0.9713 & 0.9713 \\
\hline 50 & 17 & 51 & 0.5510 & 0.3643 & 91.88 & 50.83 & 0.9694 & 0.9694 \\
\hline
\end{tabular}


$\mathrm{S}_{\text {base }}=100$ and $V_{\text {base }}=22 \mathrm{kV}$

The total active power load $=1924.0500 \mathrm{~kW}$

The total reactive power load $=1060.3600 \mathrm{kVAr}$

The total active power losses $=34.2918 \mathrm{~kW}$

The total reactive power losses $=47.5025 \mathrm{kVAr}$

The minimum voltage value in p.u. $=0.969$

The minimum voltage is at bus 19 


\subsection{9-bus radial system}

This system is composed of 69 buses and 68 branches as shown in Figure 17. The bus data, line data and load flow solution of this system are given in Table 17 and they are driven from (Baran and Wu 1989c).

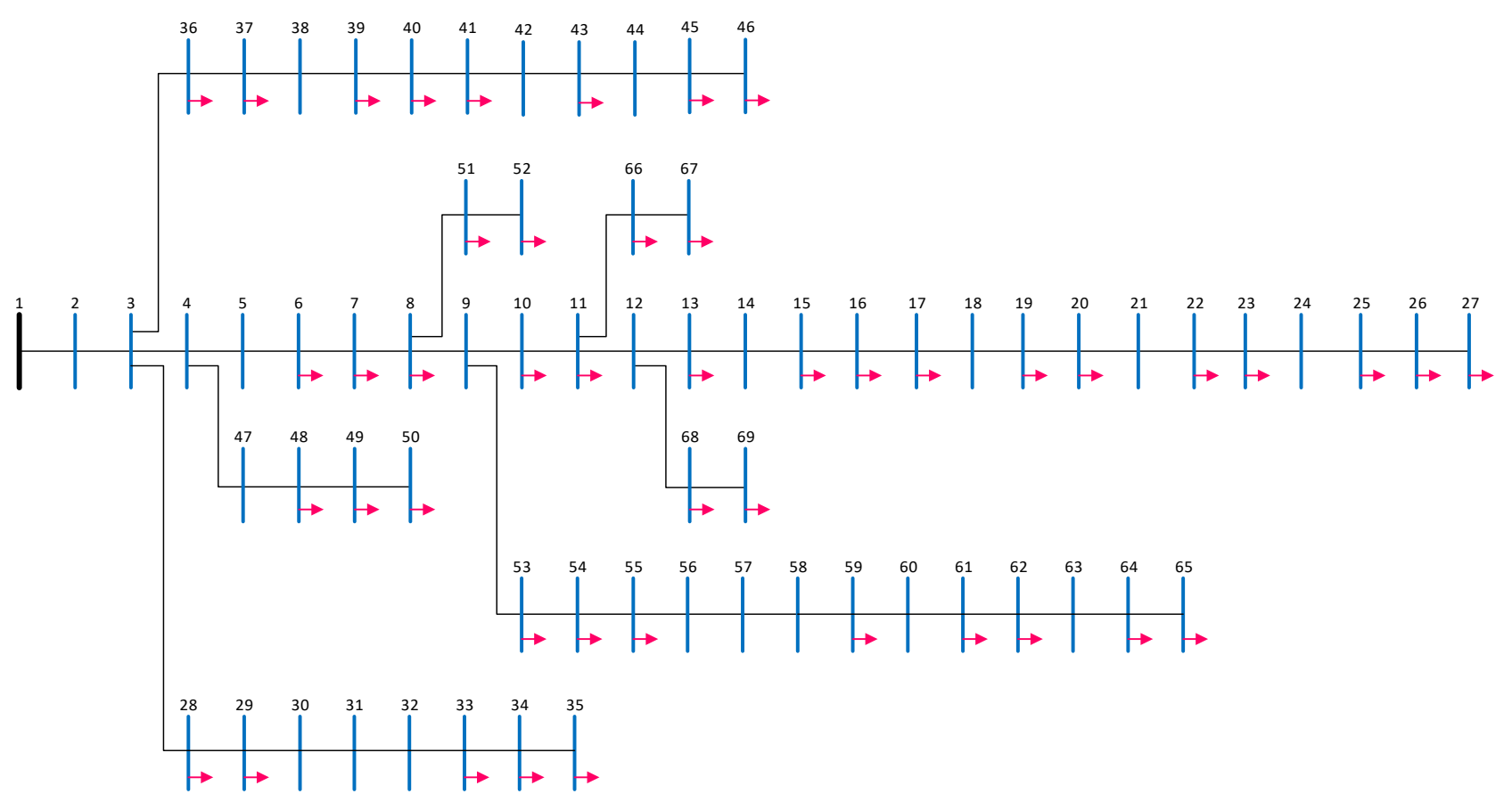

Figure 17 : Single line diagram of the 69-bus radial system. 
Table 17 : Data and load flow solution for the 69-bus radial system.

\begin{tabular}{|c|c|c|c|c|c|c|c|c|}
\hline $\begin{array}{c}\text { Branch } \\
\mathbf{N}^{\circ}\end{array}$ & $\begin{array}{c}\text { Bus } \\
\text { From }\end{array}$ & $\begin{array}{c}\text { Bus } \\
\text { To }\end{array}$ & $\begin{array}{c}\mathbf{R} \\
\text { (p.u.) }\end{array}$ & $\begin{array}{c}\mathbf{X} \\
\text { (p.u.) }\end{array}$ & $\begin{array}{c}\text { PL } \\
\text { (Bus To) } \\
\text { (kW) }\end{array}$ & $\begin{array}{c}\text { QL } \\
\text { (Bus To) } \\
\text { (kVAR) }\end{array}$ & $\begin{array}{c}\mathrm{V} \\
\text { (Bus To) } \\
\text { (pu) } \\
\text { NR } \\
\end{array}$ & $\begin{array}{c}\mathbf{V} \\
\text { (Bus To) } \\
\text { (pu) } \\
\text { PQSUM } \\
\end{array}$ \\
\hline 1 & 1 & 2 & 0.0000 & 0.0001 & 0.00 & 0.00 & 1.0000 & 1.0000 \\
\hline 2 & 2 & 3 & 0.0000 & 0.0001 & 0.00 & 0.00 & 0.9999 & 0.9999 \\
\hline 3 & 3 & 4 & 0.0001 & 0.0002 & 0.00 & 0.00 & 0.9998 & 0.9998 \\
\hline 4 & 4 & 5 & 0.0016 & 0.0018 & 0.00 & 0.00 & 0.9990 & 0.9990 \\
\hline 5 & 5 & 6 & 0.0228 & 0.0116 & 2.60 & 2.20 & 0.9901 & 0.9901 \\
\hline 6 & 6 & 7 & 0.0238 & 0.0121 & 40.40 & 30.00 & 0.9808 & 0.9808 \\
\hline 7 & 7 & 8 & 0.0058 & 0.0029 & 75.00 & 54.00 & 0.9786 & 0.9786 \\
\hline 8 & 8 & 9 & 0.0031 & 0.0016 & 30.00 & 22.00 & 0.9774 & 0.9774 \\
\hline 9 & 9 & 10 & 0.0511 & 0.0169 & 28.00 & 19.00 & 0.9724 & 0.9724 \\
\hline 10 & 10 & 11 & 0.0117 & 0.0039 & 145.00 & 104.00 & 0.9713 & 0.9713 \\
\hline 11 & 11 & 12 & 0.0444 & 0.0147 & 145.00 & 104.00 & 0.9682 & 0.9682 \\
\hline 12 & 12 & 13 & 0.0643 & 0.0212 & 8.00 & 5.50 & 0.9653 & 0.9653 \\
\hline 13 & 13 & 14 & 0.0651 & 0.0215 & 8.00 & 5.50 & 0.9624 & 0.9624 \\
\hline 14 & 14 & 15 & 0.0660 & 0.0218 & 0.00 & 0.00 & 0.9595 & 0.9595 \\
\hline 15 & 15 & 16 & 0.0123 & 0.0041 & 45.50 & 30.00 & 0.9590 & 0.9590 \\
\hline 16 & 16 & 17 & 0.0234 & 0.0077 & 60.00 & 35.00 & 0.9581 & 0.9581 \\
\hline 17 & 17 & 18 & 0.0003 & 0.0001 & 60.00 & 35.00 & 0.9581 & 0.9581 \\
\hline 18 & 18 & 19 & 0.0204 & 0.0068 & 0.00 & 0.00 & 0.9576 & 0.9576 \\
\hline 19 & 19 & 20 & 0.0131 & 0.0043 & 1.00 & 0.60 & 0.9573 & 0.9573 \\
\hline 20 & 20 & 21 & 0.0213 & 0.0070 & 114.00 & 81.00 & 0.9568 & 0.9568 \\
\hline 21 & 21 & 22 & 0.0009 & 0.0003 & 5.30 & 3.50 & 0.9568 & 0.9568 \\
\hline 22 & 22 & 23 & 0.0099 & 0.0033 & 0.00 & 0.00 & 0.9567 & 0.9567 \\
\hline 23 & 23 & 24 & 0.0216 & 0.0071 & 28.00 & 20.00 & 0.9566 & 0.9566 \\
\hline 24 & 24 & 25 & 0.0467 & 0.0154 & 0.00 & 0.00 & 0.9564 & 0.9564 \\
\hline 25 & 25 & 26 & 0.0193 & 0.0064 & 14.00 & 10.00 & 0.9563 & 0.9563 \\
\hline 26 & 26 & 27 & 0.0108 & 0.0036 & 14.00 & 10.00 & 0.9563 & 0.9563 \\
\hline 27 & 3 & 28 & 0.0003 & 0.0007 & 26.00 & 18.60 & 0.9999 & 0.9999 \\
\hline 28 & 28 & 29 & 0.0040 & 0.0098 & 26.00 & 18.60 & 0.9999 & 0.9999 \\
\hline 29 & 29 & 30 & 0.0248 & 0.0082 & 0.00 & 0.00 & 0.9997 & 0.9997 \\
\hline 30 & 30 & 31 & 0.0044 & 0.0014 & 0.00 & 0.00 & 0.9997 & 0.9997 \\
\hline 31 & 31 & 32 & 0.0219 & 0.0072 & 0.00 & 0.00 & 0.9996 & 0.9996 \\
\hline 32 & 32 & 33 & 0.0523 & 0.0176 & 14.00 & 10.00 & 0.9993 & 0.9993 \\
\hline 33 & 33 & 34 & 0.1066 & 0.0352 & 19.50 & 14.00 & 0.9990 & 0.9990 \\
\hline 34 & 34 & 35 & 0.0920 & 0.0304 & 6.00 & 4.00 & 0.9989 & 0.9989 \\
\hline 35 & 3 & 36 & 0.0003 & 0.0007 & 26.00 & 18.60 & $\begin{array}{l}0.99999 \\
\end{array}$ & 0.9999 \\
\hline 36 & 36 & 37 & 0.0040 & 0.0098 & 26.00 & 18.60 & 0.9997 & 0.9997 \\
\hline 37 & 37 & 38 & 0.0066 & 0.0077 & 0.00 & 0.00 & 0.9996 & 0.9996 \\
\hline 38 & 38 & 39 & 0.0019 & 0.0022 & 24.00 & 17.00 & 0.9995 & 0.9995 \\
\hline 39 & 39 & 40 & 0.0001 & 0.0001 & 24.00 & 17.00 & 0.9995 & 0.9995 \\
\hline 40 & 40 & 41 & 0.0454 & 0.0531 & 1.20 & 1.00 & 0.9988 & 0.9988 \\
\hline 41 & 41 & 42 & 0.0193 & 0.0226 & 0.00 & 0.00 & 0.9986 & 0.9986 \\
\hline 42 & 42 & 43 & 0.0026 & 0.0030 & 6.00 & 4.30 & 0.9985 & 0.9985 \\
\hline 43 & 43 & 44 & 0.0006 & 0.0007 & 0.00 & 0.00 & 0.9985 & 0.9985 \\
\hline 44 & 44 & 45 & 0.0068 & 0.0086 & 39.20 & 26.30 & 0.9984 & 0.9984 \\
\hline 45 & 45 & 46 & 0.0001 & 0.0001 & 39.20 & 26.30 & 0.9984 & 0.9984 \\
\hline 46 & 4 & 47 & 0.0002 & 0.0005 & 0.00 & 0.00 & 0.9998 & 0.9998 \\
\hline 47 & 47 & 48 & 0.0053 & 0.0130 & $\begin{array}{l}79.00 \\
\end{array}$ & 56.40 & 0.9985 & 0.9985 \\
\hline 48 & 48 & 49 & 0.0181 & 0.0442 & 384.70 & 274.50 & 0.9947 & 0.9947 \\
\hline 49 & 49 & 50 & 0.0051 & 0.0125 & 384.70 & 274.50 & 0.9942 & 0.9942 \\
\hline 50 & 8 & 51 & 0.0058 & 0.0030 & 40.50 & 28.30 & 0.9785 & 0.9785 \\
\hline
\end{tabular}




\begin{tabular}{|l|c|c|c|c|c|c|c|c|}
51 & 51 & 52 & 0.0207 & 0.0070 & 3.60 & 2.70 & 0.9785 & 0.9785 \\
\hline 52 & 9 & 53 & 0.0109 & 0.0055 & 4.30 & 3.50 & 0.9747 & 0.9747 \\
\hline 53 & 53 & 54 & 0.0127 & 0.0065 & 26.40 & 19.00 & 0.9714 & 0.9714 \\
\hline 54 & 54 & 55 & 0.0177 & 0.0090 & 24.00 & 17.20 & 0.9669 & 0.9669 \\
\hline 55 & 55 & 56 & 0.0176 & 0.0089 & 0.00 & 0.00 & 0.9626 & 0.9626 \\
\hline 56 & 56 & 57 & 0.0992 & 0.0333 & 0.00 & 0.00 & 0.9401 & 0.9401 \\
\hline 57 & 57 & 58 & 0.0489 & 0.0164 & 0.00 & 0.00 & 0.9290 & 0.9290 \\
\hline 58 & 58 & 59 & 0.0190 & 0.0063 & 100.00 & 72.00 & 0.9248 & 0.9248 \\
\hline 59 & 59 & 60 & 0.0241 & 0.0073 & 0.00 & 0.00 & 0.9197 & 0.9197 \\
\hline 60 & 60 & 61 & 0.0317 & 0.0161 & 1244.00 & 888.00 & 0.9123 & 0.9123 \\
\hline 61 & 61 & 62 & 0.0061 & 0.0031 & 32.00 & 23.00 & 0.9120 & 0.9120 \\
\hline 62 & 62 & 63 & 0.0090 & 0.0046 & 0.00 & 0.00 & 0.9117 & 0.9117 \\
\hline 63 & 63 & 64 & 0.0443 & 0.0226 & 227.00 & 162.00 & 0.9098 & 0.9098 \\
\hline 64 & 64 & 65 & 0.0650 & 0.0331 & 59.00 & 42.00 & 0.9092 & 0.9092 \\
\hline 65 & 11 & 66 & 0.0126 & 0.0038 & 18.00 & 13.00 & 0.9713 & 0.9713 \\
\hline 66 & 66 & 67 & 0.0003 & 0.0001 & 18.00 & 13.00 & 0.9713 & 0.9713 \\
\hline 67 & 12 & 68 & 0.0461 & 0.0152 & 28.00 & 20.00 & 0.9678 & 0.9678 \\
\hline 68 & 68 & 69 & 0.0003 & 0.0001 & 28.00 & 20.00 & 0.9678 & 0.9678 \\
\hline
\end{tabular}

$\mathrm{S}_{\text {base }}=100$ and $V_{\text {base }}=12.66 \mathrm{kV}$

The total active power load $=3802.1000 \mathrm{~kW}$

The total reactive power load $=2694.7000 \mathrm{kVAr}$

The total active power losses $=225.0007 \mathrm{~kW}$

The total reactive power losses $=102.1648 \mathrm{kVAr}$

The minimum voltage value in p.u. $=0.909$

The minimum voltage is at bus 65 


\subsection{0-bus radial system}

This system is composed of 74 buses, 73 branches, two substations and four feeders, as shown in Figure 18. The bus data, line data and load flow solution of this system are given in Table 18 and they are driven from (Das 2006).

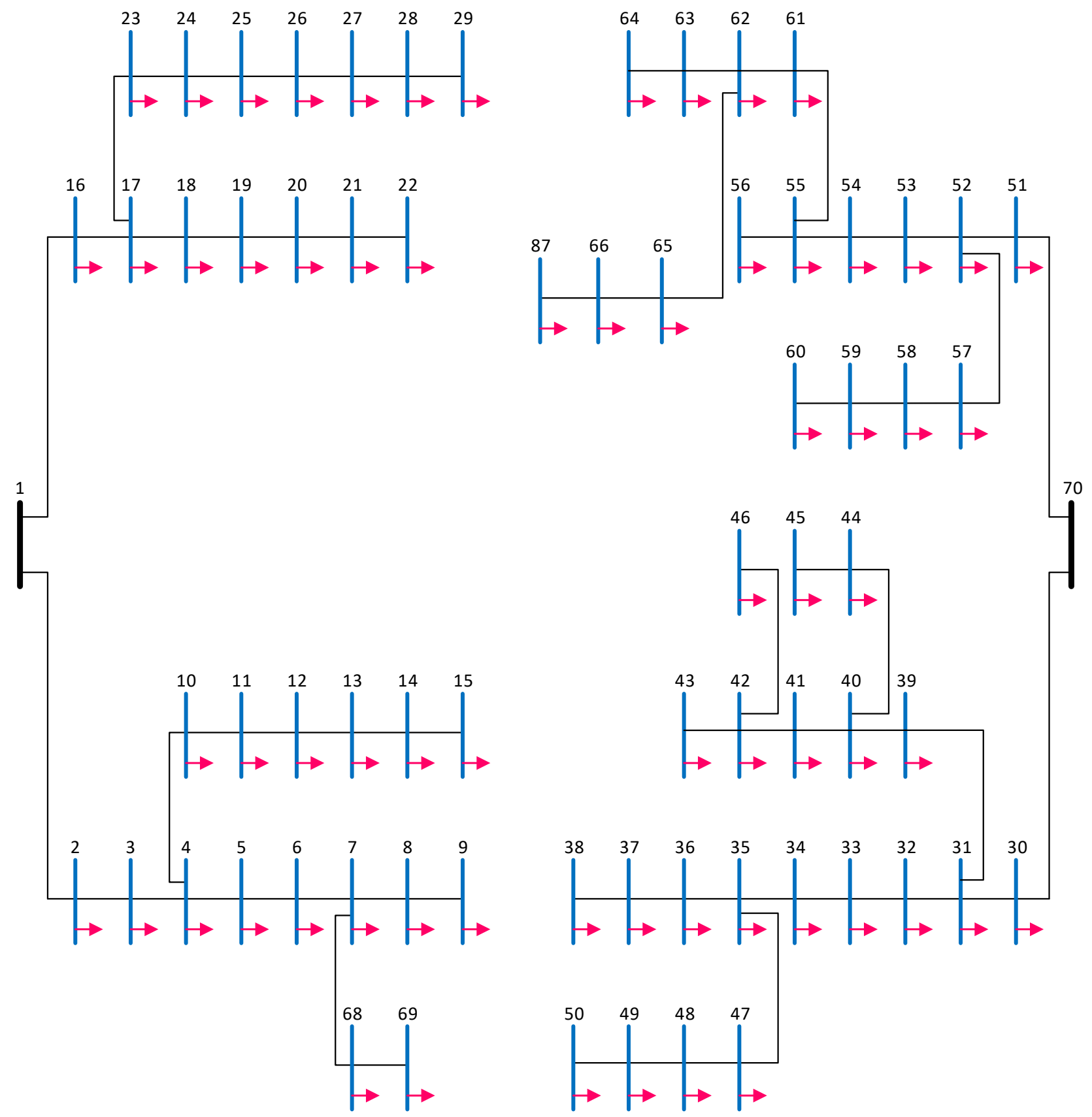

Figure 18 : Single line diagram of the 70-bus radial system. 
Table 18 : Data and load flow solution for the 70-bus radial system.

\begin{tabular}{|c|c|c|c|c|c|c|c|c|}
\hline $\begin{array}{c}\text { Branch } \\
\mathbf{N}^{\circ}\end{array}$ & $\begin{array}{c}\text { Bus } \\
\text { From }\end{array}$ & $\begin{array}{c}\text { Bus } \\
\text { To }\end{array}$ & $\begin{array}{c}\mathbf{R} \\
\text { (p.u.) }\end{array}$ & $\begin{array}{c}\mathbf{X} \\
\text { (p.u.) }\end{array}$ & $\begin{array}{c}\text { PL } \\
\text { (Bus To) } \\
(\mathbf{k W})\end{array}$ & $\begin{array}{c}\text { QL } \\
\text { (Bus To) } \\
\text { (kVAR) }\end{array}$ & $\begin{array}{c}\mathbf{V} \\
\text { (Bus To) } \\
\text { (pu) } \\
\text { NR } \\
\end{array}$ & $\begin{array}{c}\mathbf{V} \\
\text { (Bus To) } \\
\text { (pu) } \\
\text { PQSUM } \\
\end{array}$ \\
\hline 1 & 1 & 2 & 0.9066 & 0.8876 & 120.00 & 108.00 & 0.9832 & 0.9832 \\
\hline 2 & 2 & 3 & 1.2091 & 1.1835 & 72.00 & 48.00 & 0.9636 & 0.9636 \\
\hline 3 & 3 & 4 & 0.6041 & 0.5917 & 180.00 & 156.00 & 0.9545 & 0.9545 \\
\hline 4 & 4 & 5 & 0.3025 & 0.2959 & 90.00 & 60.00 & 0.9527 & 0.9527 \\
\hline 5 & 5 & 6 & 1.5107 & 1.4793 & 21.60 & 13.00 & 0.9462 & 0.9462 \\
\hline 6 & 6 & 7 & 0.9066 & 0.8876 & 21.60 & 17.00 & 0.9426 & 0.9426 \\
\hline 7 & 7 & 8 & 0.6041 & 0.5917 & 15.60 & 12.00 & 0.9423 & 0.9423 \\
\hline 8 & 8 & 9 & 0.6041 & 0.5917 & 19.00 & 13.00 & 0.9420 & 0.9420 \\
\hline 9 & 4 & 10 & 0.8926 & 0.6066 & 24.00 & 12.00 & 0.9501 & 0.9501 \\
\hline 10 & 10 & 11 & 1.3388 & 0.9099 & 19.20 & 11.00 & 0.9440 & 0.9440 \\
\hline 11 & 11 & 12 & 0.8926 & 0.6066 & 60.00 & 48.00 & 0.9401 & 0.9401 \\
\hline 12 & 12 & 13 & 1.1157 & 0.7579 & 126.00 & 108.00 & 0.9364 & 0.9364 \\
\hline 13 & 13 & 14 & 0.6694 & 0.4545 & 30.00 & 18.00 & 0.9356 & 0.9356 \\
\hline 14 & 14 & 15 & 1.6066 & 1.0917 & 48.00 & 30.00 & 0.9345 & 0.9345 \\
\hline 15 & 7 & 68 & 0.8926 & 0.6066 & 120.00 & 72.00 & 0.9403 & 0.9403 \\
\hline 16 & 68 & 69 & 1.3388 & 0.9099 & 48.00 & 36.00 & 0.9393 & 0.9393 \\
\hline 17 & 1 & 16 & 0.9066 & 0.8876 & 72.00 & 36.00 & 0.9819 & 0.9819 \\
\hline 18 & 16 & 17 & 0.3025 & 0.2959 & 48.00 & 30.00 & 0.9762 & 0.9762 \\
\hline 19 & 17 & 18 & 1.2091 & 1.1835 & 18.00 & 11.00 & 0.9715 & 0.9715 \\
\hline 20 & 18 & 19 & 0.7554 & 0.7397 & 15.60 & 8.40 & 0.9688 & 0.9688 \\
\hline 21 & 19 & 20 & 0.6645 & 0.6504 & 36.00 & 24.00 & 0.9666 & 0.9666 \\
\hline 22 & 20 & 21 & 0.9364 & 0.9174 & 108.00 & 60.00 & 0.9641 & 0.9641 \\
\hline 23 & 21 & 22 & 0.3926 & 0.3843 & 60.00 & 36.00 & 0.9637 & 0.9637 \\
\hline 24 & 17 & 23 & 1.8298 & 1.2438 & 72.00 & 48.00 & 0.9534 & 0.9534 \\
\hline 25 & 23 & 24 & 1.3388 & 0.9174 & 120.00 & 96.00 & 0.9382 & 0.9382 \\
\hline 26 & 24 & 25 & 0.8926 & 0.6066 & 96.00 & 78.00 & 0.9298 & 0.9298 \\
\hline 27 & 25 & 26 & 0.4463 & 0.3033 & 120.00 & 72.00 & 0.9263 & 0.9263 \\
\hline 28 & 26 & 27 & 0.4463 & 0.3033 & 120.00 & 66.00 & 0.9236 & 0.9236 \\
\hline 29 & 27 & 28 & 0.8926 & 0.6066 & 144.00 & 84.00 & 0.9199 & 0.9199 \\
\hline 30 & 28 & 29 & 0.8926 & 0.6066 & 126.00 & 84.00 & 0.9181 & 0.9181 \\
\hline 31 & 70 & 30 & 0.3025 & 0.2959 & 96.00 & 60.00 & 0.9909 & 0.9909 \\
\hline 32 & 30 & 31 & 0.6041 & 0.5917 & 72.00 & 48.00 & 0.9735 & 0.9735 \\
\hline 33 & 31 & 32 & 0.6041 & 0.5917 & 15.60 & 9.60 & 0.9570 & 0.9570 \\
\hline 34 & 32 & 33 & 0.6645 & 0.6504 & 19.20 & 11.80 & 0.9473 & 0.9473 \\
\hline 35 & 33 & 34 & 0.9669 & 0.9463 & 60.00 & 36.00 & 0.9335 & 0.9335 \\
\hline 36 & 34 & 35 & 0.6347 & 0.6215 & 48.00 & 33.60 & 0.9251 & 0.9251 \\
\hline 37 & 35 & 36 & 0.6041 & 0.5917 & 72.00 & 48.00 & 0.9233 & 0.9233 \\
\hline 38 & 36 & 37 & 0.9066 & 0.8876 & 48.00 & 36.00 & 0.9218 & 0.9218 \\
\hline 39 & 37 & 38 & 1.2091 & 1.1835 & 36.00 & 30.00 & 0.9210 & 0.9210 \\
\hline 40 & 32 & 39 & 0.8926 & 0.6066 & 180.00 & 120.00 & 0.9471 & 0.9471 \\
\hline 41 & 39 & 40 & 0.4463 & 0.3033 & 72.00 & 42.00 & 0.9434 & 0.9434 \\
\hline 42 & 40 & 41 & 0.8926 & 0.6066 & 144.00 & 84.00 & 0.9387 & 0.9387 \\
\hline 43 & 41 & 42 & 1.5174 & 1.0314 & 108.00 & 72.00 & 0.9339 & 0.9339 \\
\hline 44 & 42 & 43 & 1.0711 & 0.7281 & 21.60 & 12.00 & 0.9336 & 0.9336 \\
\hline 45 & 40 & 44 & 0.9818 & 0.6669 & 19.20 & 12.00 & 0.9414 & 0.9414 \\
\hline 46 & 44 & 45 & 0.4463 & 0.3033 & 120.00 & 60.00 & 0.9406 & 0.9406 \\
\hline 47 & 42 & 46 & 0.8926 & 0.6066 & 72.00 & 48.00 & 0.9329 & 0.9329 \\
\hline 48 & 35 & 47 & 0.4463 & 0.3033 & 108.00 & 84.00 & 0.9213 & 0.9213 \\
\hline 49 & 47 & 48 & 0.8926 & 0.6066 & 122.40 & 79.20 & 0.9155 & 0.9155 \\
\hline 50 & 48 & 49 & 0.8926 & 0.6066 & 120.00 & 84.00 & 0.9114 & 0.9114 \\
\hline
\end{tabular}




\begin{tabular}{|l|l|l|l|l|l|l|l|l|}
51 & 49 & 50 & 0.8926 & 0.6066 & 168.00 & 108.00 & 0.9090 & 0.9090 \\
\hline 52 & 70 & 51 & 0.3025 & 0.2959 & 72.00 & 48.00 & 0.9916 & 0.9916 \\
\hline 53 & 51 & 52 & 1.2091 & 1.1835 & 24.00 & 13.20 & 0.9597 & 0.9597 \\
\hline 54 & 52 & 53 & 1.2091 & 1.1835 & 48.00 & 36.00 & 0.9396 & 0.9396 \\
\hline 55 & 53 & 54 & 0.7554 & 0.7397 & 43.20 & 28.80 & 0.9278 & 0.9278 \\
\hline 56 & 54 & 55 & 0.9066 & 0.8876 & 36.00 & 24.00 & 0.9142 & 0.9142 \\
\hline 57 & 55 & 56 & 0.9066 & 0.8876 & 51.60 & 36.00 & 0.9134 & 0.9134 \\
\hline 58 & 52 & 57 & 0.2231 & 0.1512 & 96.00 & 60.00 & 0.9578 & 0.9578 \\
\hline 59 & 57 & 58 & 0.2231 & 0.1512 & 288.00 & 144.00 & 0.9563 & 0.9563 \\
\hline 60 & 58 & 59 & 0.6694 & 0.4545 & 150.00 & 132.00 & 0.9543 & 0.9543 \\
\hline 61 & 59 & 60 & 1.0711 & 0.7281 & 30.00 & 12.00 & 0.9539 & 0.9539 \\
\hline 62 & 55 & 61 & 0.9818 & 0.6669 & 12.00 & 6.00 & 0.9030 & 0.9030 \\
\hline 63 & 61 & 62 & 0.9818 & 0.6669 & 180.00 & 156.00 & 0.8918 & 0.8918 \\
\hline 64 & 62 & 63 & 0.6694 & 0.4545 & 60.00 & 36.00 & 0.8908 & 0.8908 \\
\hline 65 & 63 & 64 & 1.3388 & 0.9099 & 36.00 & 24.00 & 0.8900 & 0.8900 \\
\hline 66 & 62 & 65 & 0.8926 & 0.6066 & 156.00 & 144.00 & 0.8860 & 0.8860 \\
\hline 67 & 65 & 66 & 0.4463 & 0.3033 & 180.00 & 156.00 & 0.8843 & 0.8843 \\
\hline 68 & 66 & 67 & 0.8926 & 0.6066 & 30.00 & 18.00 & 0.8839 & 0.8839 \\
\hline
\end{tabular}

$S_{\text {base }}=100$ and $V_{\text {base }}=11 \mathrm{kV}$

The total active power load $=5385.4000 \mathrm{~kW}$

The total reactive power load $=3687.6000 \mathrm{kVAr}$

The total active power losses $=341.4270 \mathrm{~kW}$

The total reactive power losses $=307.5841 \mathrm{kVAr}$

The minimum voltage value in p.u. $=0.884$

The minimum voltage is at bus 67 


\subsection{4-bus radial system}

This system is composed of 70 buses, 68 branches, two substations and four feeders, as shown in Figure 19. The bus data, line data and load flow solution of this system are given in Table 19 and they are driven from (Myint and Naing 2015).

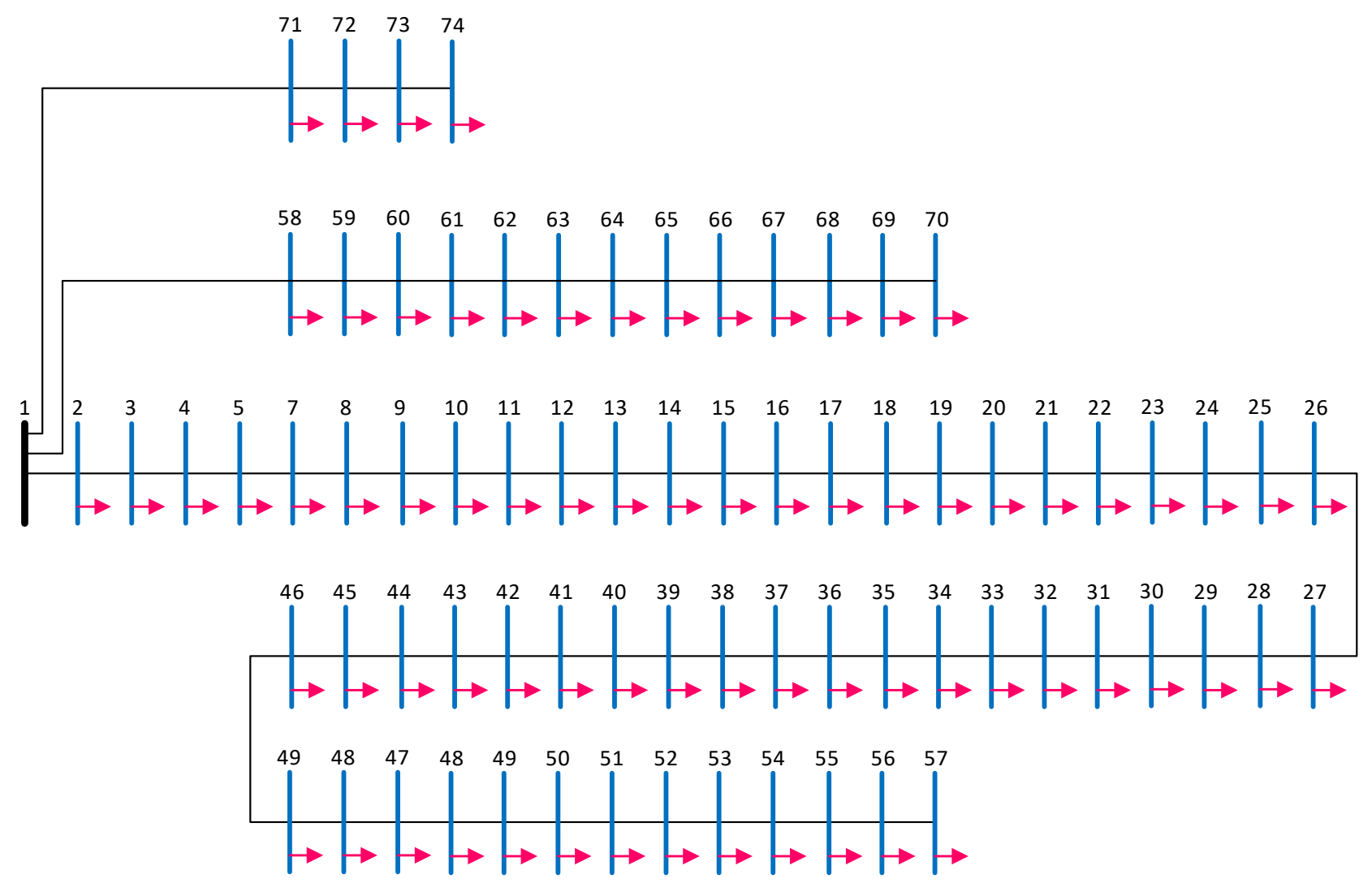

Figure 19 : Single line diagram of the 74-bus radial system. 
Table 19 : Data and load flow solution for the 74-bus radial system.

\begin{tabular}{|c|c|c|c|c|c|c|c|c|}
\hline $\begin{array}{c}\text { Branch } \\
\mathbf{N}^{\circ}\end{array}$ & $\begin{array}{c}\text { Bus } \\
\text { From }\end{array}$ & $\begin{array}{c}\text { Bus } \\
\text { To }\end{array}$ & $\begin{array}{c}\mathbf{R} \\
\text { (p.u.) }\end{array}$ & $\begin{array}{c}\mathbf{X} \\
\text { (p.u.) }\end{array}$ & $\begin{array}{c}\text { PL } \\
\text { (Bus To) } \\
\text { (kW) }\end{array}$ & $\begin{array}{c}\text { QL } \\
\text { (Bus To) } \\
\text { (kVAR) }\end{array}$ & $\begin{array}{c}\mathbf{V} \\
\text { (Bus To) } \\
\text { (pu) } \\
\text { NR } \\
\end{array}$ & $\begin{array}{c}\mathbf{V} \\
\text { (Bus To) } \\
\text { (pu) } \\
\text { PQSUM } \\
\end{array}$ \\
\hline 1 & 1 & 2 & 0.0478 & 0.0362 & 193.00 & 130.00 & 0.9976 & 0.9976 \\
\hline 2 & 2 & 3 & 0.0466 & 0.0354 & 1.00 & 1.00 & 0.9954 & 0.9954 \\
\hline 3 & 3 & 4 & 0.0356 & 0.0270 & 3.00 & 2.00 & 0.9937 & 0.9937 \\
\hline 4 & 4 & 5 & 0.0356 & 0.0270 & 108.00 & 73.00 & 0.9921 & 0.9921 \\
\hline 5 & 5 & 6 & 0.0298 & 0.0226 & 5.00 & 3.00 & 0.9907 & 0.9907 \\
\hline 6 & 6 & 7 & 0.0298 & 0.0226 & 18.00 & 12.00 & 0.9894 & 0.9894 \\
\hline 7 & 7 & 8 & 0.0239 & 0.0181 & 16.00 & 11.00 & $\begin{array}{l}0.9883 \\
\end{array}$ & 0.9883 \\
\hline 8 & 8 & 9 & 0.0239 & 0.0181 & 76.00 & 51.00 & 0.9872 & 0.9872 \\
\hline 9 & 9 & 10 & 0.0239 & 0.0181 & 52.00 & 35.00 & 0.9862 & 0.9862 \\
\hline 10 & 10 & 11 & 0.0239 & 0.0181 & 46.00 & 31.00 & 0.9852 & 0.9852 \\
\hline 11 & 11 & 12 & 0.0239 & 0.0181 & 123.00 & 83.00 & 0.9842 & 0.9842 \\
\hline 12 & 12 & 13 & 0.0239 & 0.0181 & 46.00 & 31.00 & 0.9832 & 0.9832 \\
\hline 13 & 13 & 14 & 0.0239 & 0.0181 & 7.00 & 4.00 & 0.9823 & 0.9823 \\
\hline 14 & 14 & 15 & 0.0239 & 0.0181 & 70.00 & 47.00 & 0.9813 & 0.9813 \\
\hline 15 & 15 & 16 & 0.0384 & 0.0291 & 70.00 & 47.00 & 0.9799 & 0.9799 \\
\hline 16 & 16 & 17 & 0.0384 & 0.0291 & 4.00 & 3.00 & 0.9784 & 0.9784 \\
\hline 17 & 17 & 18 & 0.0384 & 0.0291 & 49.00 & 33.00 & 0.9770 & 0.9770 \\
\hline 18 & 18 & 19 & 0.0384 & 0.0291 & 57.00 & 38.00 & 0.9756 & 0.9756 \\
\hline 19 & 19 & 20 & 0.0333 & 0.0252 & 118.00 & 79.00 & 0.9744 & 0.9744 \\
\hline 20 & 20 & 21 & 0.0333 & 0.0252 & 19.00 & 13.00 & 0.9733 & 0.9733 \\
\hline 21 & 21 & 22 & 0.0333 & 0.0252 & 14.00 & 10.00 & 0.9722 & 0.9722 \\
\hline 22 & 22 & 23 & 0.0333 & 0.0252 & 8.00 & 6.00 & 0.9711 & 0.9711 \\
\hline 23 & 23 & 24 & 0.0419 & 0.0317 & 210.00 & 141.00 & 0.9697 & 0.9697 \\
\hline 24 & 24 & 25 & 0.0298 & 0.0226 & 136.00 & 92.00 & 0.9688 & 0.9688 \\
\hline 25 & 25 & 26 & 0.0372 & 0.0282 & 189.00 & 127.00 & 0.9678 & 0.9678 \\
\hline 26 & 26 & 27 & 0.0372 & 0.0282 & 16.00 & 10.00 & 0.9669 & 0.9669 \\
\hline 27 & 27 & 28 & 0.0372 & 0.0282 & 74.00 & 49.00 & 0.9660 & 0.9660 \\
\hline 28 & 28 & 29 & 0.0239 & 0.0181 & 117.00 & 79.00 & 0.9654 & 0.9654 \\
\hline 29 & 29 & 30 & 0.0239 & 0.0181 & 6.00 & 4.00 & 0.9649 & 0.9649 \\
\hline 30 & 30 & 31 & 0.0356 & 0.0270 & 125.00 & 84.00 & 0.9641 & 0.9641 \\
\hline 31 & 31 & 32 & 0.0333 & 0.0252 & 17.00 & 11.00 & 0.9635 & 0.9635 \\
\hline 32 & 32 & 33 & 0.0298 & 0.0226 & 4.00 & 3.00 & 0.9629 & 0.9629 \\
\hline 33 & 33 & 34 & 0.0356 & 0.0270 & 19.00 & 13.00 & 0.9622 & 0.9622 \\
\hline 34 & 34 & 35 & 0.0384 & 0.0291 & 31.00 & 21.00 & 0.9615 & 0.9615 \\
\hline 35 & 35 & 36 & 0.0384 & 0.0291 & 40.00 & 27.00 & 0.9608 & 0.9608 \\
\hline 36 & 36 & 37 & 0.0400 & 0.0302 & 11.00 & 7.00 & 0.9601 & 0.9601 \\
\hline 37 & 37 & 38 & 0.0400 & 0.0302 & 217.00 & 146.00 & 0.9594 & 0.9594 \\
\hline 38 & 38 & 39 & 0.0356 & 0.0270 & 37.00 & 25.00 & 0.9589 & 0.9589 \\
\hline 39 & 39 & 40 & 0.0356 & 0.0270 & 114.00 & 77.00 & 0.9584 & 0.9584 \\
\hline 40 & 40 & 41 & 0.0298 & 0.0226 & 12.00 & 8.00 & 0.9581 & 0.9581 \\
\hline 41 & 41 & 42 & 0.0298 & 0.0226 & 55.00 & 37.00 & 0.9577 & 0.9577 \\
\hline 42 & 42 & 43 & 0.0478 & 0.0362 & 72.00 & 48.00 & 0.9572 & 0.9572 \\
\hline 43 & 43 & 44 & 0.0384 & 0.0291 & 40.00 & 27.00 & 0.9568 & 0.9568 \\
\hline 44 & 44 & 45 & 0.0419 & 0.0317 & 4.00 & 3.00 & 0.9565 & 0.9565 \\
\hline 45 & 45 & 46 & 0.0443 & 0.0336 & 41.00 & 27.00 & 0.9561 & 0.9561 \\
\hline 46 & 46 & 47 & 0.0454 & 0.0345 & 42.00 & 28.00 & 0.9557 & 0.9557 \\
\hline 47 & 47 & 48 & 0.0466 & 0.0354 & 49.00 & 33.00 & 0.9554 & 0.9554 \\
\hline 48 & 48 & 49 & 0.0349 & 0.0264 & 43.00 & 29.00 & 0.9551 & 0.9551 \\
\hline 49 & 49 & 50 & 0.0356 & 0.0270 & 61.00 & 41.00 & 0.9549 & 0.9549 \\
\hline 50 & 50 & 51 & 0.0536 & 0.0407 & 46.00 & 31.00 & 0.9546 & 0.9546 \\
\hline
\end{tabular}




\begin{tabular}{|l|c|c|c|c|c|c|c|c|}
51 & 51 & 52 & 0.0513 & 0.0389 & 10.00 & 7.00 & 0.9544 & 0.9544 \\
\hline 52 & 52 & 53 & 0.0525 & 0.0398 & 9.00 & 6.00 & 0.9542 & 0.9542 \\
\hline 53 & 53 & 54 & 0.0525 & 0.0398 & 65.00 & 44.00 & 0.9540 & 0.9540 \\
\hline 54 & 54 & 55 & 0.0525 & 0.0398 & 45.00 & 30.00 & 0.9538 & 0.9538 \\
\hline 55 & 55 & 56 & 0.0263 & 0.0199 & 76.00 & 51.00 & 0.9538 & 0.9538 \\
\hline 56 & 56 & 57 & 0.0263 & 0.0199 & 71.00 & 48.00 & 0.9537 & 0.9537 \\
\hline 57 & 1 & 58 & 0.1661 & 0.1259 & 394.00 & 265.00 & 0.9926 & 0.9926 \\
\hline 58 & 58 & 59 & 0.0596 & 0.0451 & 234.00 & 157.00 & 0.9903 & 0.9903 \\
\hline 59 & 59 & 60 & 0.0596 & 0.0451 & 227.00 & 153.00 & 0.9882 & 0.9882 \\
\hline 60 & 60 & 61 & 0.0596 & 0.0451 & 225.00 & 151.00 & 0.9863 & 0.9863 \\
\hline 61 & 61 & 62 & 0.0596 & 0.0451 & 192.00 & 129.00 & 0.9846 & 0.9846 \\
\hline 62 & 62 & 63 & 0.0596 & 0.0451 & 118.00 & 79.00 & 0.9831 & 0.9831 \\
\hline 63 & 63 & 64 & 0.0596 & 0.0451 & 418.00 & 281.00 & 0.9817 & 0.9817 \\
\hline 64 & 64 & 65 & 0.0596 & 0.0451 & 234.00 & 157.00 & 0.9807 & 0.9807 \\
\hline 65 & 65 & 66 & 0.0596 & 0.0451 & 221.00 & 148.00 & 0.9798 & 0.9798 \\
\hline 66 & 66 & 67 & 0.0596 & 0.0451 & 198.00 & 133.00 & 0.9792 & 0.9792 \\
\hline 67 & 67 & 68 & 0.0596 & 0.0451 & 249.00 & 167.00 & 0.9788 & 0.9788 \\
\hline 68 & 68 & 69 & 0.0596 & 0.0451 & 113.00 & 76.00 & 0.9786 & 0.9786 \\
\hline 69 & 69 & 70 & 0.0596 & 0.0451 & 96.00 & 64.00 & 0.9785 & 0.9785 \\
\hline 70 & 1 & 71 & 0.3918 & 0.2969 & 91.00 & 61.00 & 0.9971 & 0.9971 \\
\hline 71 & 71 & 72 & 0.1960 & 0.1485 & 116.00 & 78.00 & 0.9959 & 0.9959 \\
\hline 72 & 72 & 73 & 0.1371 & 0.1040 & 168.00 & 113.00 & 0.9953 & 0.9953 \\
\hline 73 & 73 & 74 & 0.0885 & 0.0671 & 116.00 & 78.00 & 0.9951 & 0.9951 \\
\hline
\end{tabular}

$S_{\text {base }}=100$ and $V_{\text {base }}=11 \mathrm{kV}$

The total active power load $=6617.0000 \mathrm{~kW}$

The total reactive power load $=4447.0000 \mathrm{kVAr}$

The total active power losses $=145.1363 \mathrm{~kW}$

The total reactive power losses $=109.9673 \mathrm{kVAr}$

The minimum voltage value in p.u. $=0.954$

The minimum voltage is at bus 57 


\subsection{5-bus radial system}

This system is composed of 85 buses, 84 branches as shown in Figure 20. The bus data, line data and load flow solution of this system are given in Table 20 and they are driven from (Das et al. 1995).

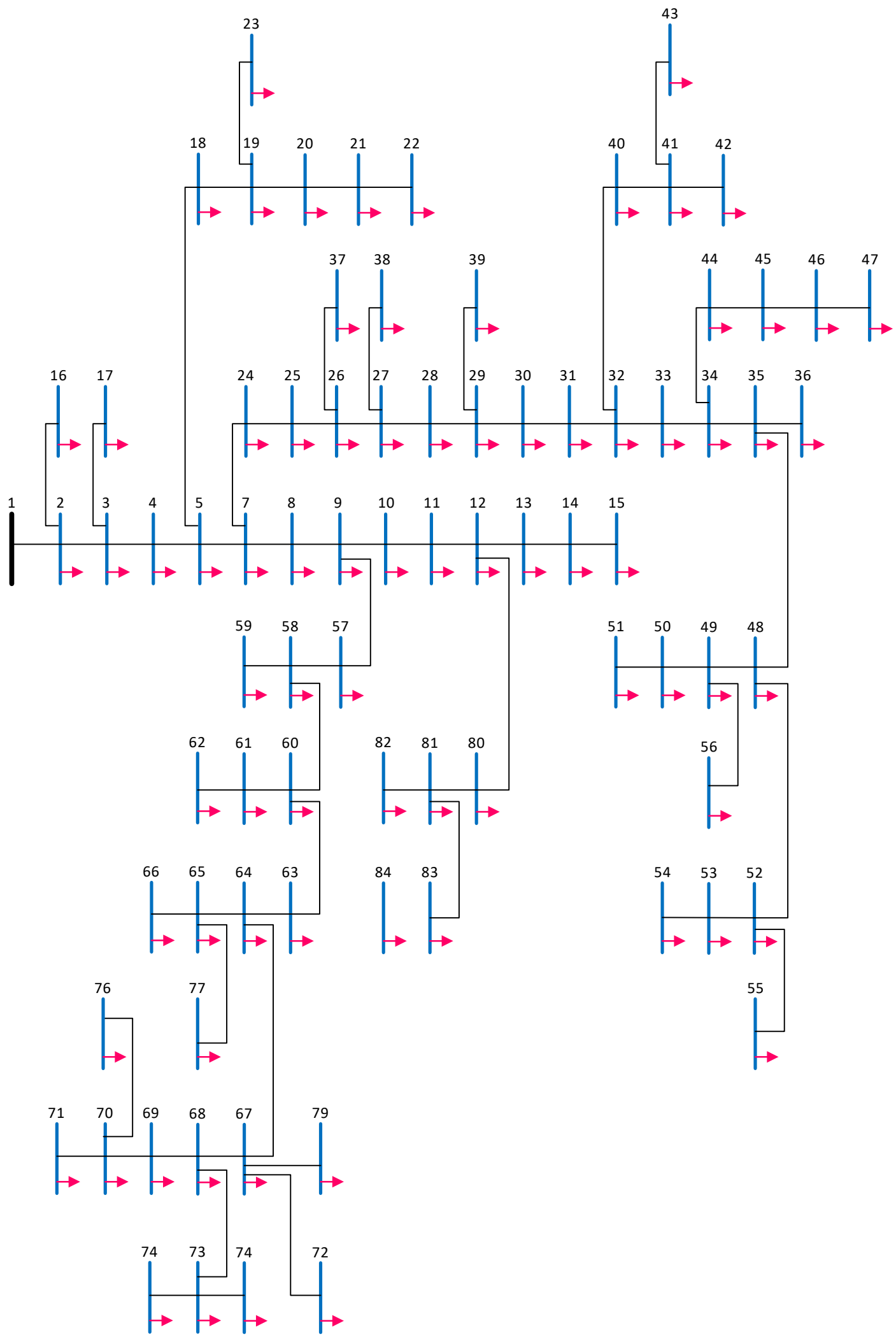

Figure 20 : Single line diagram of the 85 -bus radial system. 
Table 20 : Data and load flow solution for the 85-bus radial system.

\begin{tabular}{|c|c|c|c|c|c|c|c|c|}
\hline $\begin{array}{c}\text { Branch } \\
\mathbf{N}^{\circ}\end{array}$ & $\begin{array}{l}\text { Bus } \\
\text { From }\end{array}$ & $\begin{array}{c}\text { Bus } \\
\text { To }\end{array}$ & $\begin{array}{c}\mathbf{R} \\
\text { (p.u.) }\end{array}$ & $\begin{array}{c}\mathbf{X} \\
\text { (p.u.) }\end{array}$ & $\begin{array}{c}\text { PL } \\
\text { (Bus To) } \\
(\mathbf{k W})\end{array}$ & $\begin{array}{c}\text { QL } \\
\text { (Bus To) } \\
\text { (kVAR) }\end{array}$ & $\begin{array}{c}\mathbf{V} \\
\text { (Bus To) } \\
\text { (pu) } \\
\text { NR } \\
\end{array}$ & $\begin{array}{c}\mathrm{V} \\
\text { (Bus To) } \\
\text { (pu) } \\
\text { PQSUM } \\
\end{array}$ \\
\hline 1 & 1 & 2 & 0.0893 & 0.0620 & 0 & 0 & 0.9958 & 0.9958 \\
\hline 2 & 2 & 3 & 0.1347 & 0.0926 & 0 & 0 & 0.9895 & 0.9895 \\
\hline 3 & 3 & 4 & 0.1793 & 0.1231 & 56 & 57.1314 & 0.9815 & 0.9815 \\
\hline 4 & 4 & 5 & 0.0893 & 0.0612 & 0 & 0 & 0.9777 & 0.9777 \\
\hline 5 & 5 & 6 & 0.3595 & 0.2463 & 35.28 & 35.9928 & 0.9638 & 0.9638 \\
\hline 6 & 6 & 7 & 0.2248 & 0.1537 & 0 & 0 & 0.9552 & 0.9552 \\
\hline 7 & 7 & 8 & 0.9893 & 0.6777 & 35.28 & 35.9928 & 0.9182 & 0.9182 \\
\hline 8 & 8 & 9 & 0.0893 & 0.0612 & 0 & 0 & 0.9165 & 0.9165 \\
\hline 9 & 9 & 10 & 0.4942 & 0.3388 & 0 & 0 & 0.9130 & 0.9130 \\
\hline 10 & 10 & 11 & 0.4496 & 0.3083 & 56 & $\begin{array}{l}57.1314 \\
\end{array}$ & 0.9103 & 0.9103 \\
\hline 11 & 11 & 12 & 0.4496 & 0.3083 & 0 & 0 & 0.9080 & 0.9080 \\
\hline 12 & 12 & 13 & 0.4942 & 0.3388 & 0 & 0 & 0.9071 & 0.9071 \\
\hline 13 & 13 & 14 & 0.2248 & 0.1537 & 35.28 & 35.9928 & 0.9068 & 0.9068 \\
\hline 14 & 14 & 15 & 0.2694 & 0.1843 & 35.28 & 35.9928 & 0.9066 & 0.9066 \\
\hline 15 & 2 & 16 & 0.6017 & 0.2496 & 35.28 & 35.9928 & 0.9955 & 0.9955 \\
\hline 16 & 3 & 17 & 0.3760 & 0.1562 & 112 & 114.2629 & 0.9889 & 0.9889 \\
\hline 17 & 5 & 18 & 0.6777 & 0.2810 & 56 & 57.1314 & 0.9750 & 0.9750 \\
\hline 18 & 18 & 19 & 0.5264 & 0.2182 & 56 & 57.1314 & 0.9733 & 0.9733 \\
\hline 19 & 19 & 20 & 0.3760 & 0.1562 & 35.28 & 35.9928 & 0.9727 & 0.9727 \\
\hline 20 & 20 & 21 & 0.6769 & 0.2810 & 35.28 & 35.9928 & 0.9720 & 0.9720 \\
\hline 21 & 21 & 22 & 1.2793 & 0.5306 & 35.28 & 35.9928 & 0.9713 & 0.9713 \\
\hline 22 & 19 & 23 & 0.1504 & 0.0620 & 56 & 57.1314 & 0.9732 & 0.9732 \\
\hline 23 & 7 & 24 & 0.7521 & 0.3124 & 35.28 & 35.9928 & 0.9548 & 0.9548 \\
\hline 24 & 8 & 25 & 0.3760 & 0.1562 & 35.28 & 35.9928 & 0.9127 & 0.9127 \\
\hline 25 & 25 & 26 & 0.3008 & 0.1248 & 56 & 57.1314 & 0.9084 & 0.9084 \\
\hline 26 & 26 & 27 & 0.4512 & 0.1868 & 0 & 0 & 0.9028 & 0.9028 \\
\hline 27 & 27 & 28 & 0.2256 & 0.0934 & 56 & 57.1314 & 0.9003 & 0.9003 \\
\hline 28 & 28 & 29 & 0.4512 & 0.1868 & 0 & 0 & 0.8955 & 0.8955 \\
\hline 29 & 29 & 30 & 0.4512 & 0.1868 & 35.28 & 35.9928 & 0.8911 & 0.8911 \\
\hline 30 & 30 & 31 & 0.2256 & 0.0934 & 35.28 & 35.9928 & 0.8890 & 0.8890 \\
\hline 31 & 31 & 32 & 0.1504 & 0.0620 & 0 & 0 & 0.8877 & 0.8877 \\
\hline 32 & 32 & 33 & 0.1504 & 0.0620 & 14 & 14.2829 & 0.8867 & 0.8867 \\
\hline 33 & 33 & 34 & 0.6769 & 0.2810 & 0 & 0 & 0.8822 & 0.8822 \\
\hline 34 & 34 & 35 & 0.5264 & 0.2182 & 0 & 0 & 0.8797 & 0.8797 \\
\hline 35 & 35 & 36 & 0.1504 & 0.0620 & 35.28 & 35.9928 & 0.8797 & 0.8797 \\
\hline 36 & 26 & 37 & 0.3008 & 0.1248 & 56 & 57.1314 & 0.9082 & 0.9082 \\
\hline 37 & 27 & 38 & 0.8281 & 0.3438 & 56 & 57.1314 & 0.9021 & 0.9021 \\
\hline 38 & 29 & 39 & 0.4512 & 0.1868 & 56 & 57.1314 & 0.8951 & 0.8951 \\
\hline 39 & 32 & 40 & 0.3760 & 0.1562 & 35.28 & 35.9928 & 0.8871 & 0.8871 \\
\hline 40 & 40 & 41 & 0.8281 & 0.3438 & 0 & 0 & 0.8862 & 0.8862 \\
\hline 41 & 41 & 42 & 0.2256 & 0.0934 & 35.28 & 35.9928 & 0.8860 & 0.8860 \\
\hline 42 & 41 & 43 & 0.3760 & 0.1562 & 35.28 & 35.9928 & 0.8860 & 0.8860 \\
\hline 43 & 34 & 44 & 0.8281 & 0.3438 & 35.28 & 35.9928 & 0.8806 & 0.8806 \\
\hline 44 & 44 & 45 & 0.7529 & 0.3124 & 35.28 & 35.9928 & 0.8796 & 0.8796 \\
\hline 45 & 45 & 46 & 0.7529 & 0.3124 & 35.28 & 35.9928 & 0.8790 & 0.8790 \\
\hline 46 & 46 & 47 & 0.4512 & 0.1868 & 14 & 14.2829 & 0.8789 & 0.8789 \\
\hline 47 & 35 & 48 & 0.5264 & 0.2182 & 0 & 0 & 0.8776 & 0.8776 \\
\hline 48 & 48 & 49 & 0.1504 & 0.0620 & 0 & 0 & 0.8773 & 0.8773 \\
\hline 49 & 49 & 50 & 0.3008 & 0.1248 & 36.28 & 37.013 & 0.8769 & 0.8769 \\
\hline 50 & 50 & 51 & 0.3760 & 0.1562 & 56 & 57.1314 & 0.8765 & 0.8765 \\
\hline
\end{tabular}




\begin{tabular}{|l|c|c|c|c|c|c|c|c|}
51 & 48 & 52 & 1.1289 & 0.4686 & 0 & 0 & 0.8749 & 0.8749 \\
\hline 52 & 52 & 53 & 0.3760 & 0.1562 & 35.28 & 35.9928 & 0.8743 & 0.8743 \\
\hline 53 & 53 & 54 & 0.4512 & 0.1868 & 56 & 57.1314 & 0.8739 & 0.8739 \\
\hline 54 & 52 & 55 & 0.4512 & 0.1868 & 56 & 57.1314 & 0.8744 & 0.8744 \\
\hline 55 & 49 & 56 & 0.4512 & 0.1868 & 14 & 14.2829 & 0.8772 & 0.8772 \\
\hline 56 & 9 & 57 & 0.2256 & 0.0934 & 56 & 57.1314 & 0.9142 & 0.9142 \\
\hline 57 & 57 & 58 & 0.6769 & 0.2810 & 0 & 0 & 0.9080 & 0.9080 \\
\hline 58 & 58 & 59 & 0.1504 & 0.0620 & 56 & 57.1314 & 0.9078 & 0.9078 \\
\hline 59 & 58 & 60 & 0.4512 & 0.1868 & 0 & 0 & 0.9042 & 0.9042 \\
\hline 60 & 60 & 61 & 0.6017 & 0.2496 & 56 & 57.1314 & 0.9031 & 0.9031 \\
\hline 61 & 61 & 62 & 0.8281 & 0.3430 & 56 & 57.1314 & 0.9024 & 0.9024 \\
\hline 62 & 60 & 63 & 0.1504 & 0.0620 & 14 & 14.2829 & 0.9032 & 0.9032 \\
\hline 63 & 63 & 64 & 0.6017 & 0.2496 & 0 & 0 & 0.8994 & 0.8994 \\
\hline 64 & 64 & 65 & 0.1504 & 0.0620 & 0 & 0 & 0.8992 & 0.8992 \\
\hline 65 & 65 & 66 & 0.1504 & 0.0620 & 56 & 57.1314 & 0.8991 & 0.8991 \\
\hline 66 & 64 & 67 & 0.3760 & 0.1562 & 0 & 0 & 0.8974 & 0.8974 \\
\hline 67 & 67 & 68 & 0.7521 & 0.3124 & 0 & 0 & 0.8945 & 0.8945 \\
\hline 68 & 68 & 69 & 0.9025 & 0.3744 & 56 & 57.1314 & 0.8924 & 0.8924 \\
\hline 69 & 69 & 70 & 0.3760 & 0.1562 & 0 & 0 & 0.8919 & 0.8919 \\
\hline 70 & 70 & 71 & 0.4512 & 0.1868 & 35.28 & 35.9928 & 0.8916 & 0.8916 \\
\hline 71 & 67 & 72 & 0.1504 & 0.0620 & 56 & 57.1314 & 0.8973 & 0.8973 \\
\hline 72 & 68 & 73 & 0.9785 & 0.4058 & 0 & 0 & 0.8931 & 0.8931 \\
\hline 73 & 73 & 74 & 0.2256 & 0.0934 & 56 & 57.1314 & 0.8929 & 0.8929 \\
\hline 74 & 73 & 75 & 0.8281 & 0.3438 & 35.28 & 35.9928 & 0.8927 & 0.8927 \\
\hline 75 & 70 & 76 & 0.4512 & 0.1868 & 56 & 57.1314 & 0.8915 & 0.8915 \\
\hline 76 & 65 & 77 & 0.0752 & 0.0306 & 14 & 14.2829 & 0.8992 & 0.8992 \\
\hline 77 & 10 & 78 & 0.5264 & 0.2182 & 56 & 57.1314 & 0.9126 & 0.9126 \\
\hline 78 & 67 & 79 & 0.4512 & 0.1868 & 35.28 & 35.9928 & 0.8972 & 0.8972 \\
\hline 79 & 12 & 80 & 0.6017 & 0.2496 & 56 & 57.1314 & 0.9065 & 0.9065 \\
\hline 80 & 80 & 81 & 0.3008 & 0.1248 & 0 & 0 & 0.9060 & 0.9060 \\
\hline 81 & 81 & 82 & 0.0752 & 0.0306 & 56 & 57.1314 & 0.9060 & 0.9060 \\
\hline 82 & 81 & 83 & 0.9025 & 0.3744 & 35.28 & 35.9928 & 0.9053 & 0.9053 \\
\hline 83 & 83 & 84 & 0.8281 & 0.3438 & 14 & 14.2829 & 0.9051 & 0.9051 \\
\hline 84 & 13 & 85 & 0.6769 & 0.2810 & 35.28 & 35.9928 & 0.9067 & 0.9067 \\
\hline
\end{tabular}

$\mathrm{S}_{\text {base }}=100$ and $V_{\text {base }}=11 \mathrm{kV}$

Total active power load $=2514.2800 \mathrm{~kW}$

Total reactive power load $=2565.0783 \mathrm{kVAr}$

The total active power losses $=299.3075 \mathrm{~kW}$

The total reactive power losses $=187.8123 \mathrm{kVAr}$

The minimum voltage value in p.u. $=0.874$

The minimum voltage is at bus 54 


\subsection{4-bus radial system}

This system is composed of 94 buses and 93 branches as shown in Figure 21. The bus and line data of this system are given in Table 21 and they are driven from (Pires et al. 2012).

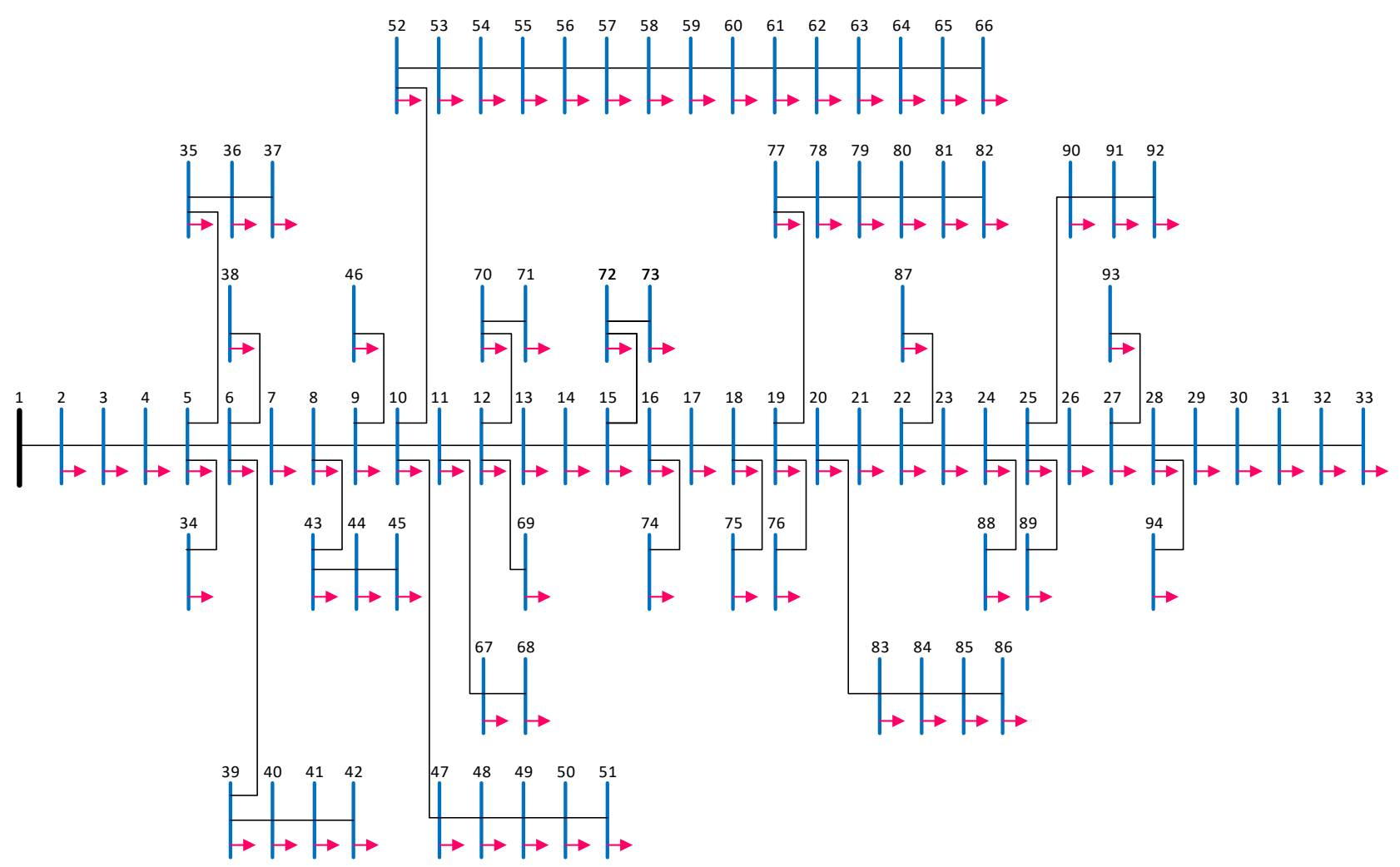

Figure 21 : Single line diagram of the 94-bus radial system. 
Table 21 : Data and load flow solution for the 94-bus radial system.

\begin{tabular}{|c|c|c|c|c|c|c|c|c|}
\hline $\begin{array}{c}\text { Branch } \\
\mathbf{N}^{\circ}\end{array}$ & $\begin{array}{c}\text { Bus } \\
\text { From }\end{array}$ & $\begin{array}{c}\text { Bus } \\
\text { To }\end{array}$ & $\begin{array}{c}\mathbf{R} \\
\text { (p.u.) }\end{array}$ & $\begin{array}{c}\mathbf{X} \\
\text { (p.u.) }\end{array}$ & $\begin{array}{c}\text { PL } \\
\text { (Bus To) } \\
\text { (kW) }\end{array}$ & $\begin{array}{c}\text { QL } \\
\text { (Bus To) } \\
\text { (kVAR) }\end{array}$ & $\begin{array}{c}\mathbf{V} \\
\text { (Bus To) } \\
\text { (pu) } \\
\text { NR } \\
\end{array}$ & $\begin{array}{c}\mathbf{V} \\
\text { (Bus To) } \\
\text { (pu) } \\
\text { PQSUM } \\
\end{array}$ \\
\hline 1 & 1 & 2 & 0.0498 & 0.0832 & 22.50 & 10.90 & 0.9951 & 0.9951 \\
\hline 2 & 2 & 3 & 0.0339 & 0.0566 & 240.30 & 116.40 & 0.9918 & 0.9918 \\
\hline 3 & 3 & 4 & 0.0840 & 0.1405 & 24.30 & 11.80 & 0.9839 & 0.9839 \\
\hline 4 & 4 & 5 & 0.0997 & 0.1666 & 0.00 & 0.00 & 0.9746 & 0.9746 \\
\hline 5 & 5 & 6 & 0.1143 & 0.1910 & 0.00 & 0.00 & 0.9645 & 0.9645 \\
\hline 6 & 6 & 7 & 0.0596 & 0.0995 & 28.80 & 14.00 & 0.9597 & 0.9597 \\
\hline 7 & 7 & 8 & 0.1327 & 0.2218 & 0.00 & 0.00 & 0.9492 & 0.9492 \\
\hline 8 & 8 & 9 & 0.0868 & 0.1451 & 0.00 & 0.00 & 0.9425 & 0.9425 \\
\hline 9 & 9 & 10 & 0.2265 & 0.3786 & 0.00 & 0.00 & 0.9252 & 0.9252 \\
\hline 10 & 10 & 11 & 0.6801 & 0.6712 & 0.00 & 0.00 & 0.9023 & 0.9023 \\
\hline 11 & 11 & 12 & 0.0840 & 0.0828 & 0.00 & 0.00 & 0.8996 & 0.8996 \\
\hline 12 & 12 & 13 & 0.0807 & 0.0797 & 0.00 & 0.00 & 0.8970 & 0.8970 \\
\hline 13 & 13 & 14 & 0.0294 & 0.0290 & 57.60 & 27.90 & 0.8961 & 0.8961 \\
\hline 14 & 14 & 15 & 0.1829 & 0.1805 & 0.00 & 0.00 & 0.8907 & 0.8907 \\
\hline 15 & 15 & 16 & 0.1148 & 0.1133 & 0.00 & 0.00 & 0.8876 & 0.8876 \\
\hline 16 & 16 & 17 & 0.0904 & 0.0892 & 18.90 & 9.20 & 0.8851 & 0.8851 \\
\hline 17 & 17 & 18 & 0.3219 & 0.3177 & 0.00 & 0.00 & 0.8766 & 0.8766 \\
\hline 18 & 18 & 19 & 0.0961 & 0.0948 & 0.00 & 0.00 & 0.8742 & 0.8742 \\
\hline 19 & 19 & 20 & 0.1556 & 0.1535 & 55.80 & 27.00 & 0.8710 & 0.8710 \\
\hline 20 & 20 & 21 & 0.6567 & 0.1729 & 40.50 & 19.60 & 0.8656 & 0.8656 \\
\hline 21 & 21 & 22 & 0.2000 & 0.0527 & 0.00 & 0.00 & 0.8641 & 0.8641 \\
\hline 22 & 22 & 23 & 0.3427 & 0.0902 & 54.00 & 26.20 & 0.8619 & 0.8619 \\
\hline 23 & 23 & 24 & 0.3933 & 0.1036 & 0.00 & 0.00 & 0.8597 & 0.8597 \\
\hline 24 & 24 & 25 & 0.4407 & 0.1160 & 0.00 & 0.00 & 0.8576 & 0.8576 \\
\hline 25 & 25 & 26 & 0.1707 & 0.0449 & 46.80 & 22.70 & 0.8571 & 0.8571 \\
\hline 26 & 26 & 27 & 0.3220 & 0.0848 & 0.00 & 0.00 & 0.8563 & 0.8563 \\
\hline 27 & 27 & 28 & 0.5267 & 0.1387 & 0.00 & 0.00 & 0.8552 & 0.8552 \\
\hline 28 & 28 & 29 & 0.5490 & 0.3066 & 13.50 & 6.50 & 0.8547 & 0.8547 \\
\hline 29 & 29 & 30 & 0.1581 & 0.0883 & 3.60 & 1.70 & 0.8546 & 0.8546 \\
\hline 30 & 30 & 31 & 0.4220 & 0.1514 & 18.00 & 8.70 & 0.8543 & 0.8543 \\
\hline 31 & 31 & 32 & 0.3066 & 0.1712 & 21.60 & 10.50 & 0.8542 & 0.8542 \\
\hline 32 & 32 & 33 & 0.6981 & 0.3899 & 9.00 & 4.40 & 0.8541 & 0.8541 \\
\hline 33 & 5 & 34 & 0.5624 & 0.2018 & 64.80 & 31.40 & 0.9742 & 0.9742 \\
\hline 34 & 5 & 35 & 0.0750 & 0.0419 & 65.70 & 31.80 & 0.9745 & 0.9745 \\
\hline 35 & 35 & 36 & 0.1218 & 0.0680 & 59.40 & 28.80 & 0.9744 & 0.9744 \\
\hline 36 & 36 & 37 & 0.1134 & 0.0633 & 13.50 & 6.50 & 0.9743 & 0.9743 \\
\hline 37 & 6 & 38 & 0.1851 & 0.1034 & 161.10 & 78.00 & 0.9641 & 0.9641 \\
\hline 38 & 6 & 39 & 0.6593 & 0.1736 & 26.10 & 12.60 & 0.9622 & 0.9622 \\
\hline 39 & 39 & 40 & 0.8000 & 0.2107 & 134.10 & 65.00 & 0.9598 & 0.9598 \\
\hline 40 & 40 & 41 & 0.2301 & 0.1285 & 85.50 & 41.40 & 0.9594 & 0.9594 \\
\hline 41 & 41 & 42 & 0.3177 & 0.1774 & 41.40 & 20.10 & 0.9592 & 0.9592 \\
\hline 42 & 8 & 43 & 0.4700 & 0.1238 & 41.40 & 20.10 & 0.9486 & 0.9486 \\
\hline 43 & 43 & 44 & 0.2310 & 0.1290 & 41.40 & 20.10 & 0.9484 & 0.9484 \\
\hline 44 & 44 & 45 & 0.1485 & 0.0829 & 21.60 & 10.50 & 0.9483 & 0.9483 \\
\hline 45 & 9 & 46 & 0.1551 & 0.0866 & 25.20 & 12.20 & 0.9424 & 0.9424 \\
\hline 46 & 10 & 47 & 0.2565 & 0.1432 & 45.90 & 22.20 & 0.9243 & 0.9243 \\
\hline 47 & 47 & 48 & 0.1599 & 0.0893 & 36.90 & 17.90 & 0.9239 & 0.9239 \\
\hline 48 & 48 & 49 & 0.3417 & 0.1908 & 63.90 & 31.00 & 0.9232 & 0.9232 \\
\hline 49 & 49 & 50 & 0.1155 & 0.0645 & 68.40 & 33.10 & 0.9230 & 0.9230 \\
\hline 50 & 50 & 51 & 0.3846 & 0.2148 & 27.90 & 13.50 & 0.9229 & 0.9229 \\
\hline
\end{tabular}




\begin{tabular}{|c|c|c|c|c|c|c|c|c|}
\hline 51 & 10 & 52 & 0.2332 & 0.2302 & 81.00 & 39.20 & 0.9196 & 0.9196 \\
\hline 52 & 52 & 53 & 0.0772 & 0.0762 & 69.30 & 33.60 & 0.9178 & 0.9178 \\
\hline 53 & 53 & 54 & 0.2732 & 0.2697 & 62.10 & 30.10 & 0.9119 & 0.9119 \\
\hline 54 & 54 & 55 & 0.0880 & 0.0868 & 35.10 & 17.00 & 0.9100 & 0.9100 \\
\hline 55 & 55 & 56 & 0.0880 & 0.0868 & 205.20 & 99.40 & 0.9083 & 0.9083 \\
\hline 56 & 56 & 57 & 0.1267 & 0.1250 & 31.50 & 15.30 & 0.9061 & 0.9061 \\
\hline 57 & 57 & 58 & 0.0635 & 0.0627 & 521.10 & 252.40 & 0.9051 & 0.9051 \\
\hline 58 & 58 & 59 & 0.1515 & 0.0846 & 212.40 & 102.90 & 0.9041 & 0.9041 \\
\hline 59 & 59 & 60 & 0.1635 & 0.0913 & 39.60 & 19.20 & 0.9035 & 0.9035 \\
\hline 60 & 60 & 61 & 0.1596 & 0.0892 & 45.00 & 21.80 & 0.9030 & 0.9030 \\
\hline 61 & 61 & 62 & 0.1557 & 0.0870 & 17.10 & 8.30 & 0.9026 & 0.9026 \\
\hline 62 & 62 & 63 & 0.1875 & 0.1047 & 21.60 & 10.50 & 0.9022 & 0.9022 \\
\hline 63 & 63 & 64 & 0.6836 & 0.2452 & 35.10 & 17.00 & 0.9009 & 0.9009 \\
\hline 64 & 64 & 65 & 0.4350 & 0.1560 & 70.20 & 34.00 & 0.9003 & 0.9003 \\
\hline 65 & 65 & 66 & 0.6627 & 0.2377 & 34.20 & 16.60 & 0.9000 & 0.9000 \\
\hline 66 & 11 & 67 & 0.4307 & 0.1134 & 22.50 & 10.90 & 0.9019 & 0.9019 \\
\hline 67 & 67 & 68 & 0.2980 & 0.0785 & 45.90 & 22.20 & 0.9018 & 0.9018 \\
\hline 68 & 12 & 69 & 0.1935 & 0.1081 & 33.30 & 16.10 & 0.8995 & 0.8995 \\
\hline 69 & 13 & 70 & 0.2058 & 0.1149 & 36.90 & 17.90 & 0.8968 & 0.8968 \\
\hline 70 & 70 & 71 & 0.1203 & 0.0672 & 45.00 & 21.80 & 0.8967 & 0.8967 \\
\hline 71 & 15 & 72 & 0.2970 & 0.1659 & 75.60 & 36.60 & 0.8901 & 0.8901 \\
\hline 72 & 72 & 73 & 0.3789 & 0.2116 & 67.50 & 32.70 & 0.8897 & 0.8897 \\
\hline 73 & 16 & 74 & 0.1473 & 0.0823 & 27.90 & 13.50 & 0.8875 & 0.8875 \\
\hline 74 & 18 & 75 & 0.1800 & 0.1005 & 38.70 & 18.70 & 0.8765 & 0.8765 \\
\hline 75 & 19 & 76 & 0.1941 & 0.1084 & 53.10 & 25.70 & 0.8740 & 0.8740 \\
\hline 76 & 19 & 77 & 0.1518 & 0.0848 & 65.70 & 31.80 & 0.8736 & 0.8736 \\
\hline 77 & 77 & 78 & 0.0939 & 0.0524 & 63.00 & 30.50 & 0.8733 & 0.8733 \\
\hline 78 & 78 & 79 & 0.5000 & 0.1793 & 67.50 & 32.70 & 0.8724 & 0.8724 \\
\hline 79 & 79 & 80 & 0.5217 & 0.2914 & 45.00 & 21.80 & 0.8718 & 0.8718 \\
\hline 80 & 80 & 81 & 0.2751 & 0.1536 & 9.00 & 4.40 & 0.8717 & 0.8717 \\
\hline 81 & 81 & 82 & 0.2526 & 0.1411 & 16.20 & 7.80 & 0.8717 & 0.8717 \\
\hline 82 & 20 & 83 & 0.3730 & 0.1338 & 67.50 & 32.70 & 0.8684 & 0.8684 \\
\hline 83 & 83 & 84 & 0.0948 & 0.0529 & 296.10 & 143.40 & 0.8678 & 0.8678 \\
\hline 84 & 84 & 85 & 0.1620 & 0.0905 & 72.00 & 34.90 & 0.8674 & 0.8674 \\
\hline 85 & 85 & 86 & 0.1425 & 0.0796 & 76.50 & 37.10 & 0.8673 & 0.8673 \\
\hline 86 & 22 & 87 & 0.3411 & 0.1905 & 90.90 & 44.00 & 0.8637 & 0.8637 \\
\hline 87 & 24 & 88 & 0.7073 & 0.2537 & 72.00 & 34.90 & 0.8590 & 0.8590 \\
\hline 88 & 25 & 89 & 0.3120 & 0.1743 & 63.00 & 30.50 & 0.8573 & 0.8573 \\
\hline 89 & 25 & 90 & 9.2191 & 0.3307 & 21.60 & 10.50 & 0.8488 & 0.8488 \\
\hline 90 & 90 & 91 & 0.3013 & 0.1081 & 36.90 & 17.90 & 0.8486 & 0.8486 \\
\hline 91 & 91 & 92 & 0.2550 & 0.1424 & 20.70 & 10.00 & 0.8485 & 0.8485 \\
\hline 92 & 27 & 93 & 0.2628 & 0.1468 & 17.10 & 8.30 & 0.8563 & 0.8563 \\
\hline 93 & 28 & 94 & 0.5273 & 0.1388 & 90.00 & 43.60 & 0.8546 & 0.8546 \\
\hline
\end{tabular}

$\mathrm{S}_{\text {base }}=100$ and $V_{\text {base }}=15 \mathrm{kV}$

The total active power load $=4797.0000 \mathrm{~kW}$

The total reactive power load $=2323.9000 \mathrm{kVAr}$

The total active power losses $=362.8578 \mathrm{~kW}$

The total reactive power losses $=504.0420 \mathrm{kVAr}$

The minimum voltage value in p.u. $=0.848$

The minimum voltage is at bus 92 


\subsection{8-bus radial system}

This system is composed of 118 buses and 117 branches as shown in Figure 22. The bus and line data of this system are given in Table 22 and they are driven from (Zhang et al. 2007).

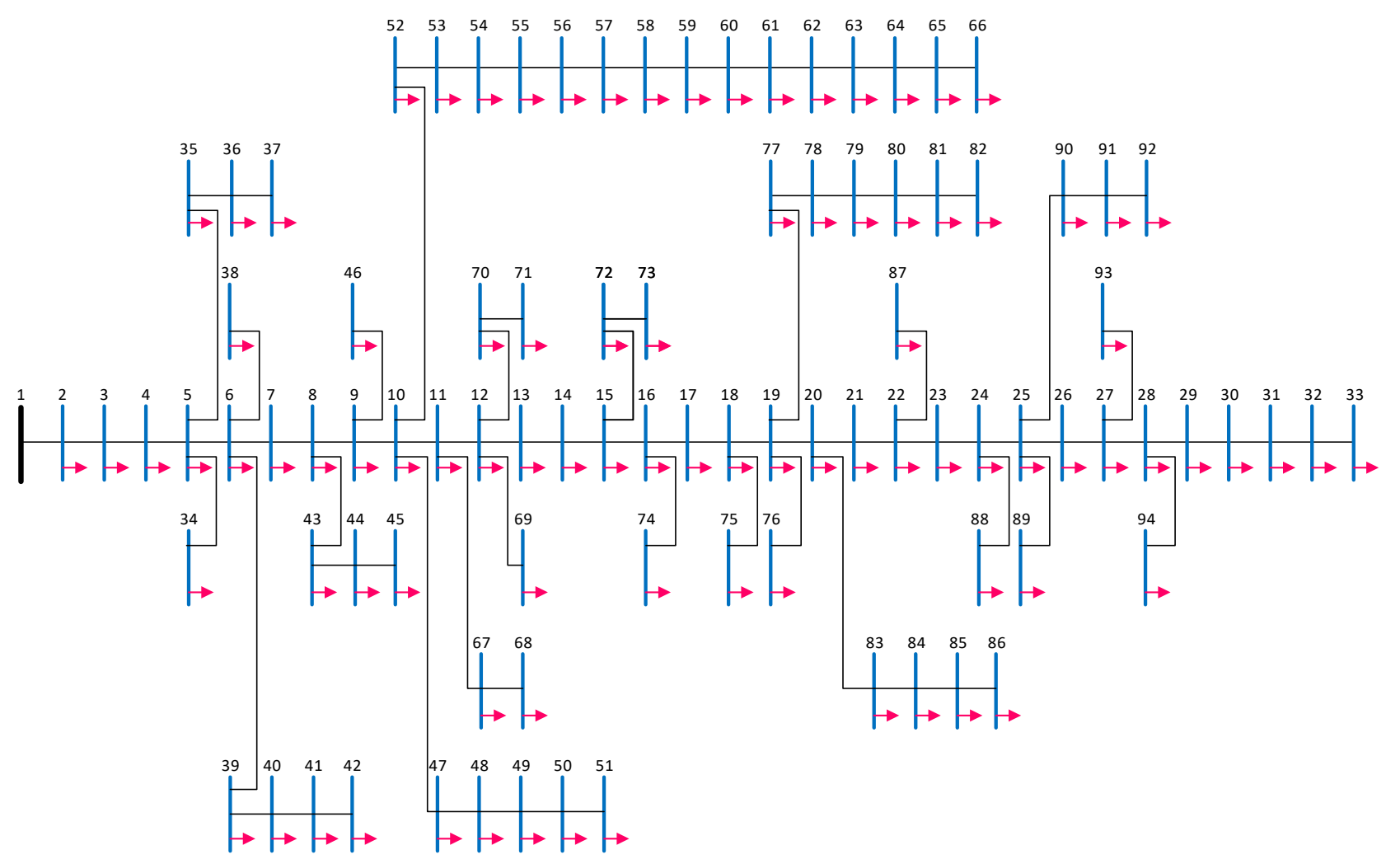

Figure 22 : Single line diagram of the 118-bus radial system. 
Table 22 : Data and load flow solution for the 118-bus radial system.

\begin{tabular}{|c|c|c|c|c|c|c|c|c|}
\hline $\begin{array}{c}\text { Branch } \\
\mathbf{N}^{\circ}\end{array}$ & $\begin{array}{c}\text { Bus } \\
\text { From }\end{array}$ & $\begin{array}{c}\text { Bus } \\
\text { To }\end{array}$ & $\begin{array}{c}\mathbf{R} \\
\text { (p.u.) }\end{array}$ & $\begin{array}{c}\mathbf{X} \\
\text { (p.u.) }\end{array}$ & $\begin{array}{c}\text { PL } \\
\text { (Bus To) } \\
\text { (kW) }\end{array}$ & $\begin{array}{c}\text { QL } \\
\text { (Bus To) } \\
\text { (kVAR) }\end{array}$ & $\begin{array}{c}\mathbf{V} \\
\text { (Bus To) } \\
\text { (pu) } \\
\text { NR } \\
\end{array}$ & $\begin{array}{c}\mathbf{V} \\
\text { (Bus To) } \\
\text { (pu) } \\
\text { PQSUM } \\
\end{array}$ \\
\hline 1 & 1 & 2 & 0.0298 & 0.0107 & 133.84 & 101.14 & 0.9959 & 0.9959 \\
\hline 2 & 2 & 3 & 0.0273 & 0.0098 & 16.21 & 11.29 & 0.9959 & 0.9959 \\
\hline 3 & 2 & 4 & 0.0372 & 0.0134 & 34.32 & 21.85 & 0.9919 & 0.9919 \\
\hline 4 & 4 & 5 & 0.0124 & 0.0446 & 73.02 & 63.60 & 0.9917 & 0.9917 \\
\hline 5 & 5 & 6 & 0.0124 & 0.0446 & 144.20 & 68.60 & 0.9916 & 0.9916 \\
\hline 6 & 6 & 7 & 0.0124 & 0.0103 & 104.47 & 61.73 & 0.9916 & 0.9916 \\
\hline 7 & 7 & 8 & 0.0149 & 0.0116 & 28.55 & 11.50 & 0.9915 & 0.9915 \\
\hline 8 & 8 & 9 & 0.0174 & 0.0521 & 87.56 & 51.07 & 0.9915 & 0.9915 \\
\hline 9 & 2 & 10 & 0.1372 & 0.1111 & 198.20 & 106.77 & 0.9914 & 0.9914 \\
\hline 10 & 10 & 11 & 0.0926 & 0.0652 & 146.80 & 76.00 & 0.9888 & 0.9888 \\
\hline 11 & 11 & 12 & 0.1545 & 0.2587 & 26.04 & 18.69 & $\begin{array}{l}0.9877 \\
\end{array}$ & 0.9877 \\
\hline 12 & 12 & 13 & 0.1174 & 0.1250 & 52.10 & 23.22 & 0.9870 & 0.9870 \\
\hline 13 & 13 & 14 & 0.1488 & 0.0975 & 141.90 & 117.50 & 0.9865 & 0.9865 \\
\hline 14 & 14 & 15 & 0.1240 & 0.0372 & 21.87 & 28.79 & 0.9864 & 0.9864 \\
\hline 15 & 15 & 16 & 0.1322 & 0.1488 & 33.37 & 26.45 & 0.9862 & 0.9862 \\
\hline 16 & 16 & 17 & 0.1298 & 0.1413 & 32.43 & 25.23 & 0.9861 & 0.9861 \\
\hline 17 & 11 & 18 & 0.1802 & 0.2355 & 20.23 & 11.91 & 0.9837 & 0.9837 \\
\hline 18 & 18 & 19 & 0.0975 & 0.1529 & 156.94 & 78.52 & 0.9808 & 0.9808 \\
\hline 19 & 19 & 20 & 0.1322 & 0.1620 & 546.29 & 351.40 & 0.9776 & 0.9776 \\
\hline 20 & 20 & 21 & 0.0992 & 0.1562 & 180.31 & 164.20 & 0.9760 & 0.9760 \\
\hline 21 & 21 & 22 & 0.0992 & 0.0652 & 93.17 & 54.59 & 0.9752 & 0.9752 \\
\hline 22 & 22 & 23 & 1.1653 & 0.5975 & 85.18 & 39.65 & 0.9681 & 0.9681 \\
\hline 23 & 23 & 24 & 0.2421 & 0.1114 & 168.10 & 95.18 & 0.9669 & 0.9669 \\
\hline 24 & 24 & 25 & 0.1099 & 0.0860 & 125.11 & 150.22 & 0.9665 & 0.9665 \\
\hline 25 & 25 & 26 & 0.1471 & 0.1107 & 16.03 & 24.62 & 0.9664 & 0.9664 \\
\hline 26 & 26 & 27 & 0.1471 & 0.1107 & 26.03 & 24.62 & 0.9663 & 0.9663 \\
\hline 27 & 4 & 28 & 0.0124 & 0.0245 & 594.56 & 522.62 & 0.9893 & 0.9893 \\
\hline 28 & 28 & 29 & 0.0099 & 0.0228 & 120.62 & 59.12 & 0.9872 & 0.9872 \\
\hline 29 & 29 & 30 & 0.0992 & 0.2286 & 102.38 & 99.55 & 0.9734 & 0.9734 \\
\hline 30 & 30 & 31 & 0.1736 & 0.2008 & 513.40 & 318.50 & 0.9606 & 0.9606 \\
\hline 31 & 31 & 32 & 0.0992 & 0.0446 & 475.25 & 456.14 & 0.9562 & 0.9562 \\
\hline 32 & 32 & 33 & 0.1471 & 0.1934 & 151.43 & 136.79 & 0.9478 & 0.9478 \\
\hline 33 & 33 & 34 & 0.1471 & 0.1934 & 205.38 & 83.30 & 0.9398 & 0.9398 \\
\hline 34 & 34 & 35 & 0.1273 & 0.1339 & 131.60 & 93.08 & 0.9341 & 0.9341 \\
\hline 35 & 30 & 36 & 0.1545 & 0.2157 & 448.40 & 369.79 & 0.9704 & 0.9704 \\
\hline 36 & 36 & 37 & 0.1099 & 0.0818 & 440.52 & 321.64 & 0.9697 & 0.9697 \\
\hline 37 & 29 & 38 & 0.2727 & 0.1603 & 112.54 & 55.13 & 0.9808 & 0.9808 \\
\hline 38 & 38 & 39 & 0.2562 & 0.1603 & 53.96 & 39.00 & 0.9750 & 0.9750 \\
\hline 39 & 39 & 40 & 0.1074 & 0.1603 & 393.05 & 342.60 & 0.9716 & 0.9716 \\
\hline 40 & 40 & 41 & 0.2314 & 0.1240 & 326.74 & 278.56 & 0.9682 & 0.9682 \\
\hline 41 & 41 & 42 & 0.9752 & 0.7025 & 536.26 & 240.24 & 0.9576 & 0.9576 \\
\hline 42 & 42 & 43 & 0.3471 & 0.2013 & 76.25 & 66.56 & 0.9565 & 0.9565 \\
\hline 43 & 43 & 44 & 0.2231 & 0.0803 & 53.52 & 39.76 & 0.9562 & 0.9562 \\
\hline 44 & 44 & 45 & 0.2802 & 0.1009 & 40.33 & 31.96 & 0.9559 & 0.9559 \\
\hline 45 & 45 & 46 & 0.2231 & 0.1470 & 39.65 & 20.76 & 0.9557 & 0.9557 \\
\hline 46 & 35 & 47 & 0.1736 & 0.1143 & 66.20 & 42.36 & 0.9281 & 0.9281 \\
\hline 47 & 47 & 48 & 0.0992 & 0.0652 & 73.90 & 51.65 & 0.9247 & 0.9247 \\
\hline 48 & 48 & 49 & 0.1240 & 0.0816 & 114.77 & 57.97 & 0.9207 & 0.9207 \\
\hline 49 & 49 & 50 & 0.1240 & 0.0816 & 918.37 & 1205.10 & 0.9169 & 0.9169 \\
\hline 50 & 50 & 51 & 0.1983 & 0.1307 & 210.30 & 146.66 & 0.9145 & 0.9145 \\
\hline
\end{tabular}




\begin{tabular}{|c|c|c|c|c|c|c|c|c|}
\hline 51 & 51 & 52 & 0.0992 & 0.0652 & 66.68 & 56.61 & 0.9136 & 0.9136 \\
\hline 52 & 52 & 53 & 0.3347 & 0.1205 & 42.21 & 40.18 & 0.9115 & 0.9115 \\
\hline 53 & 53 & 54 & 0.3347 & 0.1205 & 433.74 & 283.41 & 0.9095 & 0.9095 \\
\hline 54 & 29 & 55 & 0.3231 & 0.1165 & 62.10 & 26.86 & 0.9836 & 0.9836 \\
\hline 55 & 55 & 56 & 0.3355 & 0.1207 & 92.46 & 88.38 & 0.9800 & 0.9800 \\
\hline 56 & 56 & 57 & 0.3355 & 0.1207 & 85.19 & 55.44 & 0.9769 & 0.9769 \\
\hline 57 & 57 & 58 & 0.5835 & 0.4513 & 345.30 & 332.40 & 0.9707 & 0.9707 \\
\hline 58 & 58 & 59 & 0.2793 & 0.1007 & 22.50 & 16.83 & 0.9697 & 0.9697 \\
\hline 59 & 59 & 60 & 0.2793 & 0.1007 & 80.55 & 49.16 & 0.9689 & 0.9689 \\
\hline 60 & 60 & 61 & 0.1711 & 0.0617 & 95.86 & 90.76 & 0.9685 & 0.9685 \\
\hline 61 & 61 & 62 & 0.2041 & 0.7374 & 62.92 & 47.70 & 0.9680 & 0.9680 \\
\hline 62 & 1 & 63 & 0.0231 & 0.0345 & 478.80 & 463.74 & 0.9963 & 0.9963 \\
\hline 63 & 63 & 64 & 0.0967 & 0.1666 & 120.94 & 52.01 & 0.9807 & 0.9807 \\
\hline 64 & 64 & 65 & 0.2107 & 0.0759 & 139.11 & 100.34 & 0.9676 & 0.9676 \\
\hline 65 & 65 & 66 & 0.1736 & 0.0627 & 391.78 & 193.50 & 0.9605 & 0.9605 \\
\hline 66 & 66 & 67 & 0.3165 & 0.1140 & 27.74 & 26.71 & 0.9492 & 0.9492 \\
\hline 67 & 67 & 68 & 0.4165 & 0.2730 & 52.81 & 25.26 & 0.9322 & 0.9322 \\
\hline 68 & 68 & 69 & 0.3355 & 0.1207 & 66.89 & 38.71 & 0.9206 & 0.9206 \\
\hline 69 & 69 & 70 & 0.7950 & 0.6289 & 467.50 & 395.14 & 0.8877 & 0.8877 \\
\hline 70 & 70 & 71 & 0.1364 & 0.0496 & 594.85 & 239.74 & 0.8840 & 0.8840 \\
\hline 71 & 71 & 72 & 0.2504 & 0.0902 & 132.50 & 84.36 & 0.8792 & 0.8792 \\
\hline 72 & 72 & 73 & 0.2504 & 0.0902 & 52.70 & 22.48 & 0.8748 & 0.8748 \\
\hline 73 & 73 & 74 & 0.1702 & 0.1190 & 869.79 & 614.78 & 0.8714 & 0.8714 \\
\hline 74 & 74 & 75 & 0.1926 & 0.0694 & 31.35 & 29.82 & 0.8706 & 0.8706 \\
\hline 75 & 75 & 76 & 0.4884 & 0.1465 & 192.39 & 122.43 & 0.8689 & 0.8689 \\
\hline 76 & 76 & 77 & 0.1041 & 0.0374 & 65.75 & 45.37 & 0.8688 & 0.8688 \\
\hline 77 & 64 & 78 & 0.4620 & 0.3047 & 238.15 & 223.22 & 0.9650 & 0.9650 \\
\hline 78 & 78 & 79 & 0.1537 & 0.1014 & 294.55 & 162.47 & 0.9604 & 0.9604 \\
\hline 79 & 79 & 80 & 0.1537 & 0.1014 & 485.57 & 437.92 & 0.9575 & 0.9575 \\
\hline 80 & 80 & 81 & 0.2149 & 0.1149 & 243.53 & 183.03 & 0.9552 & 0.9552 \\
\hline 81 & 81 & 82 & 0.1273 & 0.1223 & 243.53 & 183.03 & 0.9542 & 0.9542 \\
\hline 82 & 82 & 83 & 0.1901 & 0.1058 & 134.25 & 119.29 & 0.9536 & 0.9536 \\
\hline 83 & 83 & 84 & 0.2083 & 0.0876 & 22.71 & 27.96 & 0.9534 & 0.9534 \\
\hline 84 & 84 & 85 & 0.1488 & 0.1223 & 49.51 & 26.52 & 0.9533 & 0.9533 \\
\hline 85 & 79 & 86 & 0.1322 & 0.1504 & 383.78 & 257.16 & 0.9593 & 0.9593 \\
\hline 86 & 86 & 87 & 0.1653 & 0.1901 & 49.64 & 20.60 & 0.9591 & 0.9591 \\
\hline 87 & 87 & 88 & 0.1322 & 0.3248 & 22.47 & 11.81 & 0.9591 & 0.9591 \\
\hline 88 & 65 & 89 & 0.5529 & 0.1993 & 62.93 & 42.96 & 0.9565 & 0.9565 \\
\hline 89 & 89 & 90 & 0.2198 & 0.1014 & 30.67 & 34.93 & 0.9521 & 0.9521 \\
\hline 90 & 90 & 91 & 0.2198 & 0.1014 & 62.53 & 66.79 & 0.9477 & 0.9477 \\
\hline 91 & 91 & 92 & 0.2198 & 0.1014 & 114.57 & 81.75 & 0.9469 & 0.9469 \\
\hline 92 & 92 & 93 & 0.2198 & 0.1014 & 81.29 & 66.53 & 0.9464 & 0.9464 \\
\hline 93 & 93 & 94 & 0.1926 & 0.0950 & 31.73 & 15.96 & 0.9462 & 0.9462 \\
\hline 94 & 94 & 95 & 0.4099 & 0.1140 & 33.32 & 60.48 & 0.9460 & 0.9460 \\
\hline 95 & 91 & 96 & 0.1620 & 0.1488 & 531.28 & 224.85 & 0.9448 & 0.9448 \\
\hline 96 & 96 & 97 & 0.1620 & 0.1488 & 507.03 & 367.42 & 0.9432 & 0.9432 \\
\hline 97 & 97 & 98 & 0.1542 & 0.1008 & 26.39 & 11.70 & 0.9430 & 0.9430 \\
\hline 98 & 98 & 99 & 0.0617 & 0.2628 & 45.99 & 30.39 & 0.9429 & 0.9429 \\
\hline 99 & 1 & 100 & 0.0517 & 0.0219 & 100.66 & 47.57 & 0.9963 & 0.9963 \\
\hline 100 & 100 & 101 & 0.1240 & 0.1934 & 456.48 & 350.30 & 0.9832 & 0.9832 \\
\hline 101 & 101 & 102 & 0.1113 & 0.0734 & 522.56 & 449.29 & 0.9759 & 0.9759 \\
\hline 102 & 102 & 103 & 0.1907 & 0.0994 & 408.43 & 168.46 & 0.9658 & 0.9658 \\
\hline 103 & 103 & 104 & 0.3694 & 0.1329 & 141.48 & 134.25 & 0.9497 & 0.9497 \\
\hline 104 & 104 & 105 & 0.1349 & 0.0486 & 104.43 & 66.02 & 0.9441 & 0.9441 \\
\hline 105 & 105 & 106 & 0.2727 & 0.0818 & 96.79 & 83.65 & 0.9336 & 0.9336 \\
\hline
\end{tabular}




\begin{tabular}{|l|c|c|c|c|c|c|c|c|}
106 & 106 & 107 & 0.1289 & 0.0464 & 493.92 & 419.34 & 0.9287 & 0.9287 \\
\hline 107 & 107 & 108 & 0.3156 & 0.1136 & 225.38 & 135.88 & 0.9187 & 0.9187 \\
\hline 108 & 108 & 109 & 0.1344 & 0.0483 & 509.21 & 387.21 & 0.9148 & 0.9148 \\
\hline 109 & 109 & 110 & 0.3156 & 0.1136 & 188.50 & 173.46 & 0.9081 & 0.9081 \\
\hline 110 & 110 & 111 & 0.2021 & 0.0726 & 918.03 & 898.55 & 0.9053 & 0.9053 \\
\hline 111 & 110 & 112 & 0.1726 & 0.0622 & 305.08 & 215.37 & 0.9072 & 0.9072 \\
\hline 112 & 112 & 113 & 0.1902 & 0.0684 & 54.38 & 40.97 & 0.9071 & 0.9071 \\
\hline 113 & 100 & 114 & 0.5043 & 0.1815 & 211.14 & 192.90 & 0.9929 & 0.9929 \\
\hline 114 & 114 & 115 & 0.1542 & 0.1050 & 67.01 & 53.34 & 0.9922 & 0.9922 \\
\hline 115 & 115 & 116 & 0.3084 & 0.2033 & 162.07 & 90.32 & 0.9912 & 0.9912 \\
\hline 116 & 116 & 117 & 0.3347 & 0.3033 & 48.79 & 29.16 & 0.9908 & 0.9908 \\
\hline 117 & 117 & 118 & 0.4041 & 0.3620 & 33.90 & 18.98 & 0.9906 & 0.9906 \\
\hline
\end{tabular}

$\mathrm{S}_{\text {base }}=100$ and $V_{\text {base }}=11 \mathrm{kV}$

The total active power load $=22709.7200 \mathrm{~kW}$

The total reactive power load $=17041.0680 \mathrm{kVAr}$

The total active power losses $=1298.0916 \mathrm{~kW}$

The total reactive power losses $=978.7361 \mathrm{kVAr}$

The minimum voltage value in p.u. $=0.869$

The minimum voltage is at bus 77 


\subsection{6-bus radial system}

This system is composed of 136 buses and 135 branches as shown in Figure 23. The bus and line data of this system are given in Table 23 and they are driven from (Mantovani et al. 2000).

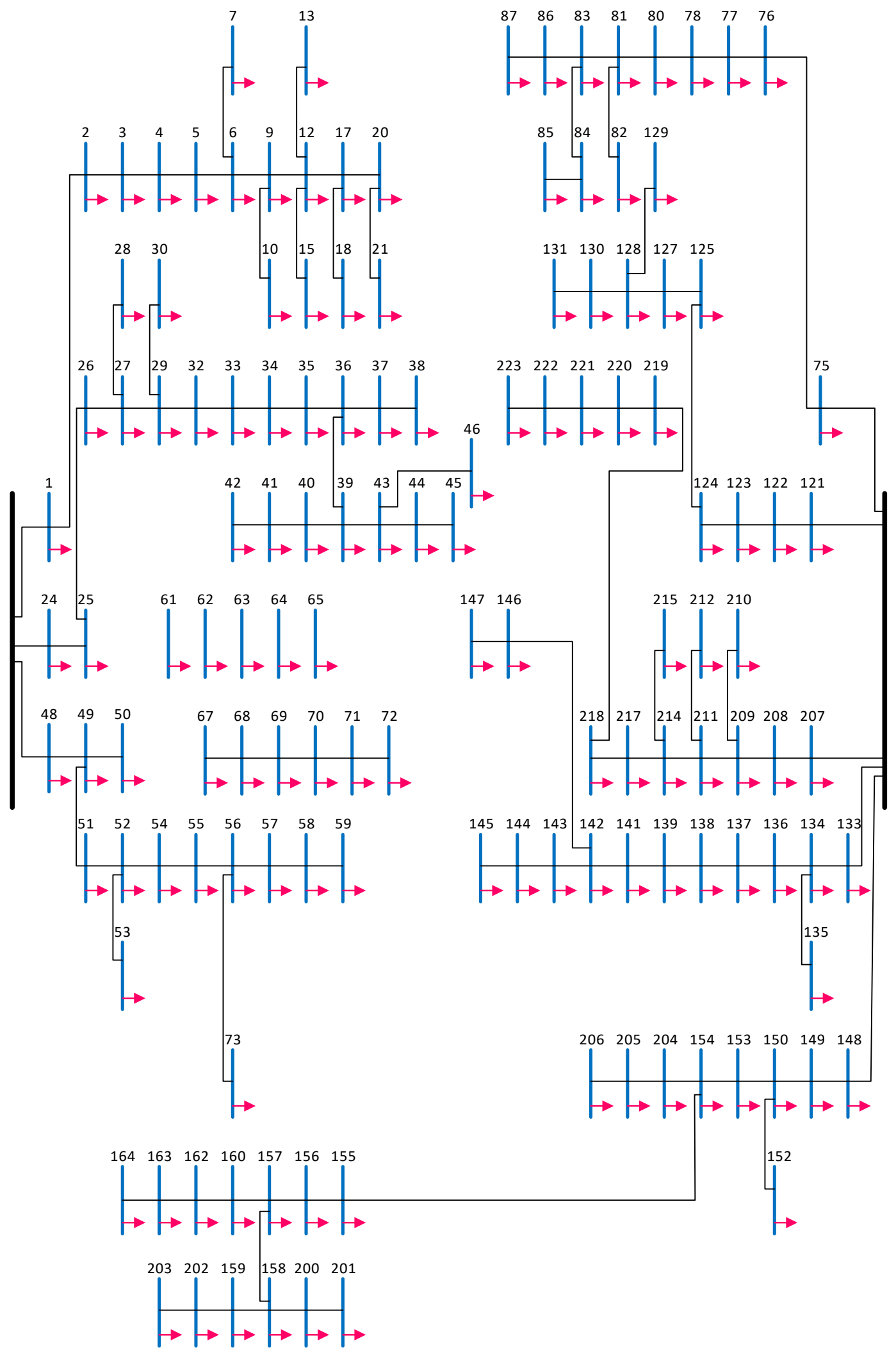

Figure 23 : Single line diagram of the 136-bus radial system. 
Table 23 : Data and load flow solution for the 136-bus radial system.

\begin{tabular}{|c|c|c|c|c|c|c|c|c|}
\hline $\begin{array}{c}\text { Branch } \\
\mathbf{N}^{\circ}\end{array}$ & $\begin{array}{c}\text { Bus } \\
\text { From }\end{array}$ & $\begin{array}{c}\text { Bus } \\
\text { To }\end{array}$ & $\begin{array}{c}\mathbf{R} \\
\text { (p.u.) }\end{array}$ & $\begin{array}{c}\mathbf{X} \\
\text { (p.u.) }\end{array}$ & $\begin{array}{c}\text { PL } \\
\text { (Bus To) } \\
\text { (kW) }\end{array}$ & $\begin{array}{c}\text { QL } \\
\text { (Bus To) } \\
\text { (kVAR) }\end{array}$ & $\begin{array}{c}\mathbf{V} \\
\text { (Bus To) } \\
\text { (pu) } \\
\text { NR } \\
\end{array}$ & $\begin{array}{c}\mathbf{V} \\
\text { (Bus To) } \\
\text { (pu) } \\
\text { PQSUM } \\
\end{array}$ \\
\hline 1 & 1 & 2 & 0.1744 & 0.4025 & 0.00 & 0.00 & 0.9910 & 0.9910 \\
\hline 2 & 2 & 3 & 0.0010 & 0.0023 & 47.78 & 19.01 & 0.9909 & 0.9909 \\
\hline 3 & 3 & 4 & 0.1172 & 0.2706 & 42.55 & 16.93 & 0.9850 & 0.9850 \\
\hline 4 & 4 & 5 & 0.0522 & 0.1205 & 87.02 & 34.62 & 0.9824 & 0.9824 \\
\hline 5 & 5 & 6 & 0.0818 & 0.1887 & 311.31 & 123.86 & 0.9785 & 0.9785 \\
\hline 6 & 6 & 7 & 0.0857 & 0.1978 & 148.87 & 59.23 & 0.9750 & 0.9750 \\
\hline 7 & 7 & 8 & 0.0601 & 0.1387 & 238.67 & 94.96 & 0.9747 & 0.9747 \\
\hline 8 & 7 & 9 & 0.0298 & 0.0298 & 62.30 & 24.79 & 0.9743 & 0.9743 \\
\hline 9 & 9 & 10 & 0.2737 & 0.1440 & 124.60 & 49.57 & 0.9738 & 0.9738 \\
\hline 10 & 9 & 11 & 0.0571 & 0.0570 & 140.18 & 55.77 & 0.9730 & 0.9730 \\
\hline 11 & 11 & 12 & 0.2090 & 0.1099 & 116.81 & 46.47 & 0.9727 & 0.9727 \\
\hline 12 & 11 & 13 & 0.4817 & 0.1652 & 249.20 & 99.15 & 0.9716 & 0.9716 \\
\hline 13 & 11 & 14 & 0.0621 & 0.0620 & 291.45 & 115.95 & 0.9721 & 0.9721 \\
\hline 14 & 14 & 15 & 0.2637 & 0.1387 & 303.72 & 120.84 & 0.9711 & 0.9711 \\
\hline 15 & 14 & 16 & 0.0298 & 0.0298 & 215.40 & 85.70 & 0.9719 & 0.9719 \\
\hline 16 & 16 & 17 & 0.1543 & 0.0811 & 198.59 & 79.01 & 0.9716 & 0.9716 \\
\hline 17 & 1 & 18 & 0.1744 & 0.4025 & 0.00 & 0.00 & 0.9913 & 0.9913 \\
\hline 18 & 18 & 19 & 0.0010 & 0.0023 & 0.00 & 0.00 & 0.9913 & 0.9913 \\
\hline 19 & 19 & 20 & 0.1172 & 0.2706 & 0.00 & 0.00 & 0.9854 & 0.9854 \\
\hline 20 & 20 & 21 & 0.0571 & 0.1319 & 30.13 & 14.73 & 0.9826 & 0.9826 \\
\hline 21 & 21 & 22 & 0.3732 & 0.1963 & 230.97 & 112.92 & 0.9815 & 0.9815 \\
\hline 22 & 21 & 23 & 0.0956 & 0.2206 & 60.26 & 29.46 & 0.9784 & 0.9784 \\
\hline 23 & 23 & 24 & 0.1592 & 0.0838 & 230.97 & 112.92 & 0.9780 & 0.9780 \\
\hline 24 & 23 & 25 & 0.0128 & 0.0296 & 120.51 & 58.92 & 0.9779 & 0.9779 \\
\hline 25 & 25 & 26 & 0.0236 & 0.0546 & 0.00 & 0.00 & 0.9771 & 0.9771 \\
\hline 26 & 26 & 27 & 0.0099 & 0.0227 & 56.98 & 27.86 & 0.9768 & 0.9768 \\
\hline 27 & 27 & 28 & 0.0621 & 0.0620 & 364.67 & 178.28 & 0.9753 & 0.9753 \\
\hline 28 & 28 & 29 & 0.0124 & 0.0124 & 0.00 & 0.00 & 0.9751 & 0.9751 \\
\hline 29 & 29 & 30 & 0.0995 & 0.0524 & 124.65 & 60.94 & 0.9749 & 0.9749 \\
\hline 30 & 30 & 31 & 0.2090 & 0.1099 & 56.98 & 27.86 & 0.9747 & 0.9747 \\
\hline 31 & 29 & 32 & 0.0298 & 0.0298 & 0.00 & 0.00 & 0.9747 & 0.9747 \\
\hline 32 & 32 & 33 & 0.0498 & 0.0262 & 85.47 & 41.79 & 0.9744 & 0.9744 \\
\hline 33 & 33 & 34 & 0.2190 & 0.1152 & 0.00 & 0.00 & 0.9732 & 0.9732 \\
\hline 34 & 34 & 35 & 0.0597 & 0.0314 & 396.74 & 193.96 & 0.9729 & 0.9729 \\
\hline 35 & 32 & 36 & 0.0397 & 0.0397 & 0.00 & 0.00 & 0.9744 & 0.9744 \\
\hline 36 & 36 & 37 & 0.1941 & 0.1021 & 181.15 & 88.56 & 0.9733 & 0.9733 \\
\hline 37 & 37 & 38 & 0.1393 & 0.0733 & 242.17 & 118.40 & 0.9729 & 0.9729 \\
\hline 38 & 36 & 39 & 0.0298 & 0.0298 & 75.32 & 36.82 & 0.9743 & 0.9743 \\
\hline 39 & 1 & 40 & 0.1744 & 0.4025 & 0.00 & 0.00 & 0.9909 & 0.9909 \\
\hline 40 & 40 & 41 & 0.0621 & 0.1433 & 1.25 & 0.53 & 0.9876 & 0.9876 \\
\hline 41 & 41 & 42 & 1.5558 & 0.5336 & 6.27 & 2.66 & 0.9875 & 0.9875 \\
\hline 42 & 41 & 43 & 0.0010 & 0.0023 & 0.00 & 0.00 & 0.9876 & 0.9876 \\
\hline 43 & 43 & 44 & 0.0364 & 0.0841 & 117.88 & 49.97 & 0.9857 & 0.9857 \\
\hline 44 & 44 & 45 & 0.4280 & 0.2251 & 62.67 & 26.57 & 0.9853 & 0.9853 \\
\hline 45 & 44 & 46 & 0.0335 & 0.0773 & 172.29 & 73.03 & 0.9841 & 0.9841 \\
\hline 46 & 46 & 47 & 0.0690 & 0.1592 & 458.56 & 194.39 & 0.9810 & 0.9810 \\
\hline 47 & 47 & 48 & 0.0325 & 0.0750 & 262.96 & 111.47 & $\begin{array}{l}0.9798 \\
\end{array}$ & 0.9798 \\
\hline 48 & 48 & 49 & 0.0601 & 0.1387 & 235.76 & 99.94 & $\begin{array}{l}0.9783 \\
\end{array}$ & 0.9783 \\
\hline 49 & 49 & 50 & 0.1490 & 0.1488 & 0.00 & 0.00 & 0.9781 & 0.9781 \\
\hline 50 & 50 & 51 & 0.1490 & 0.1488 & 109.22 & 46.30 & 0.9778 & 0.9778 \\
\hline
\end{tabular}




\begin{tabular}{|c|c|c|c|c|c|c|c|c|}
\hline 51 & 49 & 52 & 0.0236 & 0.0546 & 0.00 & 0.00 & 0.9779 & 0.9779 \\
\hline 52 & 52 & 53 & 0.0138 & 0.0318 & 72.81 & 30.87 & 0.9776 & 0.9776 \\
\hline 53 & 53 & 54 & 0.0315 & 0.0728 & 258.47 & 109.57 & 0.9774 & 0.9774 \\
\hline 54 & 54 & 55 & 0.0158 & 0.0364 & 69.17 & 29.32 & 0.9774 & 0.9774 \\
\hline 55 & 55 & 56 & 0.0108 & 0.0250 & 21.84 & 9.26 & 0.9774 & 0.9774 \\
\hline 56 & 53 & 57 & 0.0571 & 0.1319 & 0.00 & 0.00 & 0.9770 & 0.9770 \\
\hline 57 & 57 & 58 & 0.1344 & 0.0707 & 20.53 & 8.70 & 0.9762 & 0.9762 \\
\hline 58 & 58 & 59 & 0.2190 & 0.1152 & 150.55 & 63.82 & 0.9750 & 0.9750 \\
\hline 59 & 59 & 60 & 0.2637 & 0.1387 & 220.69 & 93.55 & 0.9739 & 0.9739 \\
\hline 60 & 60 & 61 & 0.1742 & 0.0916 & 92.38 & 39.16 & 0.9737 & 0.9737 \\
\hline 61 & 61 & 62 & 0.1095 & 0.0576 & 0.00 & 0.00 & 0.9737 & 0.9737 \\
\hline 62 & 48 & 63 & 0.0729 & 0.1683 & 226.69 & 96.10 & 0.9795 & 0.9795 \\
\hline 63 & 1 & 64 & 0.0039 & 0.0091 & 0.00 & 0.00 & 0.9999 & 0.9999 \\
\hline 64 & 64 & 65 & 0.1419 & 0.3275 & 294.02 & 116.97 & 0.9955 & 0.9955 \\
\hline 65 & 65 & 66 & 0.2010 & 0.4639 & 83.02 & 33.03 & 0.9906 & 0.9906 \\
\hline 66 & 66 & 67 & 0.1734 & 0.4002 & 83.02 & 33.03 & 0.9866 & 0.9866 \\
\hline 67 & 67 & 68 & 0.1724 & 0.3980 & 103.77 & 41.29 & 0.9829 & 0.9829 \\
\hline 68 & 68 & 69 & 0.0896 & 0.2069 & 176.41 & 70.18 & 0.9811 & 0.9811 \\
\hline 69 & 69 & 70 & 0.2936 & 0.1544 & 83.02 & 33.03 & 0.9808 & 0.9808 \\
\hline 70 & 69 & 71 & 0.0305 & 0.0705 & 217.92 & 86.70 & 0.9807 & 0.9807 \\
\hline 71 & 71 & 72 & 0.3683 & 0.1937 & 23.29 & 9.27 & 0.9806 & 0.9806 \\
\hline 72 & 72 & 73 & 0.5375 & 0.2827 & 5.08 & 2.02 & 0.9805 & 0.9805 \\
\hline 73 & 71 & 74 & 0.0355 & 0.0819 & 72.64 & 28.90 & 0.9804 & 0.9804 \\
\hline 74 & 74 & 75 & 0.6950 & 0.2384 & 405.99 & 161.52 & 0.9771 & 0.9771 \\
\hline 75 & 1 & 76 & 0.0059 & 0.0136 & 0.00 & 0.00 & 0.9998 & 0.9998 \\
\hline 76 & 76 & 77 & 0.3832 & 0.8846 & 100.18 & 42.47 & 0.9869 & 0.9869 \\
\hline 77 & 77 & 78 & 0.1182 & 0.2729 & 142.52 & 60.42 & 0.9832 & 0.9832 \\
\hline 78 & 78 & 79 & 0.1093 & 0.2524 & 96.04 & 40.71 & 0.9801 & 0.9801 \\
\hline 79 & 79 & 80 & 0.0246 & 0.0569 & 300.45 & 127.37 & 0.9794 & 0.9794 \\
\hline 80 & 80 & 81 & 0.3253 & 0.3248 & 141.24 & 59.87 & 0.9746 & 0.9746 \\
\hline 81 & 81 & 82 & 0.1788 & 0.1785 & 279.85 & 118.63 & 0.9724 & 0.9724 \\
\hline 82 & 82 & 83 & 0.2986 & 0.1571 & 87.31 & 37.01 & 0.9721 & 0.9721 \\
\hline 83 & 82 & 84 & 0.0571 & 0.0570 & 243.85 & 103.37 & 0.9720 & 0.9720 \\
\hline 84 & 84 & 85 & 0.2986 & 0.1571 & 247.75 & 105.03 & 0.9711 & 0.9711 \\
\hline 85 & 1 & 86 & 0.0059 & 0.0136 & 0.00 & 0.00 & 0.9997 & 0.9997 \\
\hline 86 & 86 & 87 & 0.2197 & 0.5071 & 89.88 & 38.10 & 0.9873 & 0.9873 \\
\hline 87 & 87 & 88 & 0.0551 & 0.0716 & 1137.28 & 482.11 & 0.9863 & 0.9863 \\
\hline 88 & 87 & 89 & 0.2305 & 0.5321 & 458.34 & 194.30 & 0.9801 & 0.9801 \\
\hline 89 & 89 & 90 & 0.0395 & 0.0135 & 385.20 & 163.29 & 0.9796 & 0.9796 \\
\hline 90 & 90 & 91 & 0.0404 & 0.0932 & 0.00 & 0.00 & 0.9790 & 0.9790 \\
\hline 91 & 91 & 92 & 0.1744 & 0.4025 & 79.61 & 33.75 & 0.9766 & 0.9766 \\
\hline 92 & 92 & 93 & 0.0443 & 0.1023 & 87.31 & 37.01 & 0.9760 & 0.9760 \\
\hline 93 & 93 & 94 & 0.0699 & 0.1615 & 0.00 & 0.00 & 0.9753 & 0.9753 \\
\hline 94 & 94 & 95 & 0.1540 & 0.1537 & 74.00 & 31.37 & 0.9743 & 0.9743 \\
\hline 95 & 95 & 96 & 0.1142 & 0.1141 & 232.05 & 98.37 & 0.9737 & 0.9737 \\
\hline 96 & 96 & 97 & 0.1391 & 0.1389 & 141.82 & 60.12 & 0.9734 & 0.9734 \\
\hline 97 & 94 & 98 & 0.0542 & 0.1251 & 0.00 & 0.00 & 0.9752 & 0.9752 \\
\hline 98 & 98 & 99 & 0.0709 & 0.1637 & 76.45 & 32.41 & 0.9751 & 0.9751 \\
\hline 99 & 1 & 100 & 0.0049 & 0.0114 & 0.00 & 0.00 & 0.9997 & 0.9997 \\
\hline 100 & 100 & 101 & 0.0887 & 0.2047 & 51.32 & 21.76 & 0.9939 & 0.9939 \\
\hline 101 & 101 & 102 & 0.0621 & 0.1433 & 59.87 & 25.38 & 0.9899 & 0.9899 \\
\hline 102 & 102 & 103 & 1.2004 & 0.4118 & 9.07 & 3.84 & 0.9898 & 0.9898 \\
\hline 103 & 102 & 104 & 0.2394 & 0.5526 & 2.09 & 0.89 & 0.9749 & 0.9749 \\
\hline 104 & 104 & 105 & 0.3655 & 0.8437 & 16.74 & 7.09 & 0.9525 & 0.9525 \\
\hline 105 & 105 & 106 & 0.2404 & 0.5549 & 1506.52 & 638.63 & 0.9389 & 0.9389 \\
\hline
\end{tabular}




\begin{tabular}{|l|c|c|c|c|c|c|c|c|}
106 & 106 & 107 & 0.1066 & 0.1385 & 313.02 & 132.69 & 0.9368 & 0.9368 \\
\hline 107 & 107 & 108 & 0.1121 & 0.1456 & 79.83 & 33.84 & 0.9352 & 0.9352 \\
\hline 108 & 108 & 109 & 0.2886 & 0.1518 & 51.32 & 21.76 & 0.9335 & 0.9335 \\
\hline 109 & 109 & 110 & 0.2837 & 0.1492 & 0.00 & 0.00 & 0.9325 & 0.9325 \\
\hline 110 & 108 & 111 & 0.0239 & 0.0310 & 202.44 & 85.82 & 0.9351 & 0.9351 \\
\hline 111 & 111 & 112 & 0.2488 & 0.1309 & 60.82 & 25.78 & 0.9348 & 0.9348 \\
\hline 112 & 112 & 113 & 0.4529 & 0.2382 & 45.62 & 19.34 & 0.9345 & 0.9345 \\
\hline 113 & 113 & 114 & 0.2986 & 0.1571 & 0.00 & 0.00 & 0.9345 & 0.9345 \\
\hline 114 & 109 & 115 & 0.4081 & 0.2147 & 157.07 & 66.58 & 0.9326 & 0.9326 \\
\hline 115 & 115 & 116 & 0.5673 & 0.2984 & 0.00 & 0.00 & 0.9326 & 0.9326 \\
\hline 116 & 110 & 117 & 0.5773 & 0.3036 & 250.15 & 106.04 & 0.9307 & 0.9307 \\
\hline 117 & 117 & 118 & 0.2488 & 0.1309 & 0.00 & 0.00 & 0.9307 & 0.9307 \\
\hline 118 & 105 & 119 & 0.1694 & 0.3911 & 69.81 & 29.59 & 0.9519 & 0.9519 \\
\hline 119 & 119 & 120 & 0.0768 & 0.1774 & 32.07 & 13.60 & 0.9518 & 0.9518 \\
\hline 120 & 120 & 121 & 0.0650 & 0.1501 & 61.08 & 25.89 & 0.9517 & 0.9517 \\
\hline 121 & 1 & 122 & 0.0059 & 0.0136 & 0.00 & 0.00 & 0.9997 & 0.9997 \\
\hline 122 & 122 & 123 & 0.3408 & 0.7868 & 94.62 & 46.26 & 0.9848 & 0.9848 \\
\hline 123 & 123 & 124 & 0.0236 & 0.0546 & 49.86 & 24.38 & 0.9838 & 0.9838 \\
\hline 124 & 124 & 125 & 0.2764 & 0.0948 & 123.16 & 60.21 & 0.9834 & 0.9834 \\
\hline 125 & 124 & 126 & 0.0108 & 0.0250 & 78.35 & 38.30 & 0.9834 & 0.9834 \\
\hline 126 & 126 & 127 & 0.2787 & 0.1466 & 145.48 & 71.12 & 0.9828 & 0.9828 \\
\hline 127 & 126 & 128 & 0.0512 & 0.1183 & 21.37 & 10.45 & 0.9817 & 0.9817 \\
\hline 128 & 128 & 129 & 0.0621 & 0.1433 & 74.79 & 36.56 & 0.9816 & 0.9816 \\
\hline 129 & 128 & 130 & 0.0729 & 0.1683 & 227.93 & 111.43 & 0.9794 & 0.9794 \\
\hline 130 & 130 & 131 & 0.0227 & 0.0523 & 35.61 & 17.41 & 0.9788 & 0.9788 \\
\hline 131 & 131 & 132 & 0.0483 & 0.1114 & 249.30 & 121.88 & 0.9776 & 0.9776 \\
\hline 132 & 132 & 133 & 0.0847 & 0.1956 & 316.72 & 154.84 & 0.9759 & 0.9759 \\
\hline 133 & 133 & 134 & 0.1987 & 0.1984 & 333.82 & 163.20 & 0.9742 & 0.9742 \\
\hline 134 & 134 & 135 & 0.2086 & 0.2083 & 249.30 & 121.88 & 0.9734 & 0.9734 \\
\hline 135 & 135 & 136 & 0.1540 & 0.1537 & 0.00 & 0.00 & 0.9734 & 0.9734 \\
\hline & & & & & & & & \\
\hline
\end{tabular}

$S_{\text {base }}=100$ and $V_{\text {base }}=13.8 \mathrm{kV}$

The total active power load $=18313.8070 \mathrm{~kW}$

The total reactive power load $=7932.5680 \mathrm{kVAr}$

The total active power losses $=320.3639 \mathrm{~kW}$

The total reactive power losses $=702.9474 \mathrm{kVAr}$

The minimum voltage value in p.u. $=0.931$

The minimum voltage is at bus 117

\subsection{Summary of test systems}

\section{Applications of the reviewed systems}

In Table 24 some examples of use of the presented RDS in this paper are given. In the first column of this table the name of the RDS is give, in the second and third columns the references and the year of use of the RDS are given, respectively, and in the last column the type of the study applied 
on the RDS is given. It is worth to mention that even though a large list of references is given in Table

24 it is not exhaustive and some other references can be found in the literature.

Table 24 : Some examples of use of radial test systems.

\begin{tabular}{|c|c|c|c|}
\hline Test system & References & Year & Application \\
\hline \multirow{13}{*}{ 10-bus RDS } & (Rao and Narasimham 2008) & 2008 & Optimal capacitor placement \\
\hline & (Hamouda and Zehar 2011) & 2011 & Optimal Var planning \\
\hline & (Khan et al. 2013) & 2013 & Optimal siting and sizing of shunt capacitors \\
\hline & (Baghipourr and Hosseini 2013) & 2013 & Optimal siting and sizing of DG and capacitor \\
\hline & (S. Vijayabaskar and T. Manigandan 2013) & 2013 & Analysis of RDS optimization with FACTS devices \\
\hline & (Muthukumar and Thanushkodi 2013) & 2013 & Capacitor placement \\
\hline & (Hamouda and Sayah 2013) & 2013 & Optimal capacitors sizing \\
\hline & (Elsheikh et al. 2014) & 2014 & Optimal siting and sizing of capacitors \\
\hline & (Nojavan et al. 2014) & 2014 & Optimal allocation of capacitors \\
\hline & (Peddanna 2015) & 2015 & Power factor and energy loss cost evaluation \\
\hline & (Abdelaziz et al. 2016) & 2016 & Optimal capacitor placement and sizing \\
\hline & (Murthy and Bhimaraju 2016) & 2016 & Optimal sizing of capacitors \\
\hline & (Bhongade and Arya 2016) & 2016 & Optimal siting and sizing of shunt capacitors \\
\hline \multirow{8}{*}{ 12-bus RDS } & (Hamouda and Zehar 2006) & 2006 & Load flow \\
\hline & (Gözel and Hocaoglu 2009) & 2009 & Optimal siting and sizing of DG \\
\hline & (Aman et al. 2012) & 2012 & Optimal siting and sizing of DG \\
\hline & (Kayal and Chanda 2013) & 2013 & Placement of wind and solar based DGs \\
\hline & (Gopiya Naik et al. 2013) & 2013 & Optimal allocation of combined DG and capacitor \\
\hline & (Devi and Geethanjali 2014) & 2014 & Optimal siting and sizing of DG and DSTATCOM \\
\hline & (Peddanna 2015) & 2015 & Power factor and energy loss cost evaluation \\
\hline & (Yuvaraj et al. 2017) & 2017 & Optimal allocation of DG and DSTATCOM \\
\hline \multirow{3}{*}{ 15-bus RDS } & (Kayal and Chanda 2013) & 2013 & Placement of wind and solar based DGs \\
\hline & (Elsheikh et al. 2014) & 2014 & Optimal siting and sizing of capacitors \\
\hline & (Abdelaziz et al. 2015) & 2015 & Optimal siting and sizing of capacitors \\
\hline \multirow{4}{*}{ 16-bus RDS } & (Hung et al. 2010) & 2010 & Optimal DG allocation \\
\hline & (Hung and Mithulananthan 2013) & 2013 & Optimal DG allocation \\
\hline & (Rosseti et al. 2013) & 2013 & Optimal DG allocation and network reconfiguration \\
\hline & (Lee et al. 2014) & 2014 & Network reconfiguration \\
\hline \multirow{2}{*}{ 17-bus RDS } & (Ebrahimi et al. 2013) & 2013 & Optimal siting and sizing of DG \\
\hline & (HLAING and SWE 2014) & 2014 & Optimal siting and sizing of DG \\
\hline 28-bus RDS & (Peddanna and Kishore 2015) & 2013 & Power Loss Allocation \\
\hline \multirow{12}{*}{ 33-bus RDS } & (Raoofat 2011) & 2011 & $\begin{array}{l}\text { Simultaneous allocation of DG and remote controllable } \\
\text { switches }\end{array}$ \\
\hline & (El Ramli et al. 2011) & 2011 & Dynamic network reconfiguration \\
\hline & (Moradi and Abedini 2012) & 2012 & Optimal siting and sizing of DG \\
\hline & (Ebrahimi et al. 2013) & 2013 & Optimal siting and sizing of DG \\
\hline & (Andervazh et al. 2013) & 2013 & Network reconfiguration \\
\hline & (Baghipourr and Hosseini 2013) & 2013 & Optimal siting and sizing of DG and capacitors \\
\hline & (Gopiya Naik et al. 2013) & 2013 & Optimal siting and sizing of DG and capacitors \\
\hline & (Ettehadi et al. 2013) & 2013 & Optimal DG allocation \\
\hline & (Kayal and Chanda 2013) & 2013 & Placement of wind and solar based DGs \\
\hline & (Rosseti et al. 2013) & 2013 & Optimal DG allocation and network reconfiguration \\
\hline & (Muthukumar and Thanushkodi 2013) & 2013 & Capacitor placement \\
\hline & (Lee et al. 2014) & 2014 & Network reconfiguration \\
\hline
\end{tabular}




\begin{tabular}{|c|c|c|c|}
\hline & (Mohamed Imran et al. 2014) & 2014 & Optimal DG allocation and network reconfiguration \\
\hline & (Esmaeilian and Fadaeinedjad 2014) & 2014 & Optimal DG allocation and network reconfiguration \\
\hline & (Moradi et al. 2014) & 2014 & Optimal siting and sizing of DG and shunt capacitors \\
\hline & (Bagheri Tolabi et al. 2015) & 2015 & $\begin{array}{l}\text { Network reconfiguration and optimal allocation of } \\
\text { Photovoltaic and DSTATCOM }\end{array}$ \\
\hline & (Pazouki et al. 2015) & 2015 & $\begin{array}{l}\text { Simultaneous Planning of PEV Charging Stations and } \\
\text { DGs }\end{array}$ \\
\hline & (Bakhshideh Zad et al. 2015) & 2015 & Optimal reactive power control of DGs for \\
\hline & (Kanwar et al. 2015) & 2015 & Optimal allocation of DG and capacitors \\
\hline & (Zare et al. 2015) & 2015 & Dynamic planning of DG \\
\hline & (Rajaram et al. 2015) & 2015 & Network reconfiguration \\
\hline & (Sultana and Roy 2015) & 2015 & Optimal siting of DG \\
\hline & (ChithraDevi et al. 2016) & 2016 & Optimal siting and sizing of DG \\
\hline & (Sultana and Roy 2016) & 2016 & Optimal Location of DG \\
\hline & (Dixit et al. 2017) & 2017 & Incorporation of DG and shunt capacitor \\
\hline \multirow{10}{*}{ 34-bus RDS } & (Rao and Narasimham 2008) & 2008 & Optimal capacitor placement \\
\hline & (Muthukumar and Thanushkodi 2013) & 2013 & Capacitor placement \\
\hline & (S. Vijayabaskar and T. Manigandan 2013) & 2013 & Analysis of RDS optimization with FACTS devices \\
\hline & (Elsheikh et al. 2014) & 2014 & Optimal siting and sizing of capacitors \\
\hline & (Nojavan et al. 2014) & 2014 & Optimal allocation of capacitors \\
\hline & (Devabalaji et al. 2015) & 2015 & Optimal siting and sizing of capacitors \\
\hline & (Capitanescu et al. 2015) & 2015 & Network reconfiguration \\
\hline & (Murthy and Bhimaraju 2016) & 2016 & Optimal sizing of capacitors \\
\hline & (Bhongade and Arya 2016) & 2016 & Optimal siting and sizing of shunt capacitors \\
\hline & (Yuvaraj et al. 2017) & 2017 & Optimal Allocation of DG and DSTATCOM \\
\hline \multirow{4}{*}{ 38-bus RDS } & (Abdi and Afshar 2013) & 2013 & Optimal siting and sizing of DG \\
\hline & (Shaaban et al. 2013) & 2013 & Optimal allocation of DG \\
\hline & (Mohandas et al. 2015) & 2015 & Optimal siting and sizing of DG \\
\hline & (Zhang et al. 2015) & 2015 & DG planning \\
\hline \multirow{2}{*}{ 51-bus RDS } & (Gampa and Das 2015) & 2015 & Optimal siting and sizing of DG \\
\hline & (Gampa and Das 2016) & 2016 & Optimum placement of shunt capacitors \\
\hline \multirow{18}{*}{ 69-bus RDS } & (Kashem et al. 2001) & 2001 & Network reconfiguration \\
\hline & (Acharya et al. 2006) & 2006 & Optimal siting and sizing of DG \\
\hline & (Gözel and Hocaoglu 2009) & 2009 & Optimal siting and sizing of DG \\
\hline & (Hung et al. 2010) & 2010 & Optimal DG allocation \\
\hline & (Ayres et al. 2010) & 2010 & $\begin{array}{l}\text { Determination of the maximum allowable penetration } \\
\text { level of DG }\end{array}$ \\
\hline & (Shukla et al. 2010) & 2010 & Optimal Sizing of DG \\
\hline & (Alrashidi and Alhajri 2011) & 2011 & Optimal planning of multiple DG \\
\hline & (Aman et al. 2012) & 2012 & Optimal siting and sizing of DG \\
\hline & (Moradi and Abedini 2012) & 2012 & Optimal siting and sizing of DG \\
\hline & (Khan et al. 2013) & 2013 & Optimal siting and sizing of shunt capacitors \\
\hline & (Andervazh et al. 2013) & 2013 & Network reconfiguration \\
\hline & (Injeti and Prema Kumar 2013) & 2013 & Optimal siting and sizing of DG \\
\hline & (Nekooei et al. 2013) & 2013 & Optimal siting of DG \\
\hline & (Hung and Mithulananthan 2013) & 2013 & Optimal DG allocation \\
\hline & (García and Mena 2013) & 2013 & Optimal siting and sizing of DG \\
\hline & (Kayal and Chanda 2013) & 2013 & Placement of wind and solar based DGs \\
\hline & (Kansal et al. 2013) & 2013 & Optimal siting of DG \\
\hline & (Devi and Geethanjali 2014) & 2014 & Optimal siting and sizing of DG and DSTATCOM \\
\hline
\end{tabular}




\begin{tabular}{|c|c|c|c|}
\hline & (Moradi et al. 2014) & 2014 & Optimal siting and sizing of DG and shunt capacitors \\
\hline & (Sultana and Roy 2014a) & 2014 & Optimal siting and sizing of DG \\
\hline & (Sultana and Roy 2014b) & 2014 & Optimal siting of shunt capacitor \\
\hline & (Mena and García 2015) & 2015 & Optimal siting and sizing of DG \\
\hline & (Abdelaziz et al. 2015) & 2015 & Optimal siting and sizing of capacitors \\
\hline & (El-Fergany 2015) & 2015 & Impact of load models on DG siting and sizing \\
\hline & (Sultana and Roy 2015) & 2015 & Optimal siting of DG \\
\hline & (ChithraDevi et al. 2016) & 2016 & Optimal siting and sizing of DG \\
\hline & (Abul'Wafa 2016) & 2016 & Multi-conductor feeder design \\
\hline & (Sultana and Roy 2016) & 2016 & Optimal siting of DG \\
\hline & (Gampa and Das 2016) & 2016 & Optimum placement of shunt capacitors \\
\hline & (Abdelaziz et al. 2016) & 2016 & Optimal capacitor placement and sizing \\
\hline & (Kansal et al. 2016) & 2016 & Optimal siting of DG \\
\hline & (Yuvaraj et al. 2017) & 2017 & Optimal Allocation of DG and DSTATCOM \\
\hline \multirow{4}{*}{ 70-bus RDS } & (Gupta et al. 2010) & 2010 & Network reconfiguration \\
\hline & (Niknam et al. 2012) & 2012 & Location of Automatic Voltage Regulators \\
\hline & (Azizipanah-Abarghooee et al. 2014) & 2014 & Network reconfiguration \\
\hline & (Dias et al. 2015) & 2015 & Network planning \\
\hline 74-bus RDS & (Myint 2015) & 2015 & Network reconfiguration \\
\hline \multirow{11}{*}{ 85-bus RDS } & (Rao and Narasimham 2008) & 2008 & Optimal Capacitor Placement \\
\hline & (Rao et al. 2012) & 2012 & Optimal conductor size selection \\
\hline & (Muthukumar and Thanushkodi 2013) & 2013 & Capacitor placement \\
\hline & (S. Vijayabaskar and T. Manigandan 2013) & 2013 & Analysis of RDS optimization with FACTS devices \\
\hline & (Nojavan et al. 2014) & 2014 & Optimal allocation of capacitors \\
\hline & (Sultana and Roy 2014b) & 2014 & Optimal siting of shunt capacitor \\
\hline & (Devabalaji et al. 2015) & 2015 & Optimal siting and sizing of capacitors \\
\hline & (Bhongade and Arya 2016) & 2016 & Optimal siting and sizing of shunt capacitors \\
\hline & (Abul'Wafa 2016) & 2016 & Multi-conductor feeder design \\
\hline & (Dixit et al. 2017) & 2017 & Incorporation of DG and shunt capacitor \\
\hline & (Reddy et al. 2017) & 2017 & Sizing of renewable resources \\
\hline \multirow{2}{*}{ 94-bus RDS } & (Lee et al. 2014) & 2014 & Network reconfiguration \\
\hline & (ChithraDevi et al. 2016) & 2016 & Optimal siting and sizing of DG \\
\hline \multirow{9}{*}{ 118-bus RDS } & (Injeti and Prema Kumar 2013) & 2013 & Optimal siting and sizing of DG \\
\hline & (Hemmatpour and Mohammadian 2013) & 2013 & Network reconfiguration and DG planning \\
\hline & (García and Mena 2013) & 2013 & Optimal siting and sizing of DG \\
\hline & (Sultana and Roy 2014a) & 2014 & Optimal siting and sizing of DG \\
\hline & (Mena and García 2015) & 2015 & Optimal siting and sizing of DG \\
\hline & (Abdelaziz et al. 2015) & 2015 & Optimal siting and sizing of capacitors \\
\hline & (Sultana and Roy 2015) & 2015 & Optimal siting of DG \\
\hline & (Sultana and Roy 2016) & 2016 & Optimal siting of DG \\
\hline & (Abdelaziz et al. 2016) & 2016 & Optimal capacitor placement and sizing \\
\hline \multirow{3}{*}{ 136-bus RDS } & (Gupta et al. 2010) & 2010 & Network reconfiguration \\
\hline & (El Ramli et al. 2011) & 2011 & Dynamic network reconfiguration \\
\hline & (El-Fergany 2015) & 2015 & Impact of load models on DG siting and sizing \\
\hline
\end{tabular}




\section{Conclusion}

This paper has presented a comprehensive database on the common radial distribution test systems used in majority of power system literature. This database presents a comprehensive and organized information for further research and education works purposes in the distribution system operation and planning. The important properties of the distribution system and the various application of the distribution study is provided to highlight the importance of distribution system research and study. The database and the contribution of the reviewed works are provided. We hope that this paper will help researchers, academic staffs and students in distribution system related research works.

\section{References}

Abdelaziz AY, Ali ES, Abd Elazim SM (2015) Optimal sizing and locations of capacitors in radial distribution systems via flower pollination optimization algorithm and power loss index. Eng Sci Technol an Int J 19:610-618. doi: 10.1016/j.jestch.2015.09.002

Abdelaziz AY, Ali ES, Abd Elazim SM (2016) Flower Pollination Algorithm for Optimal Capacitor Placement and Sizing in Distribution Systems. Electr Power Components Syst 44:544-555. doi: 10.1080/15325008.2015.1117540

Abdi S, Afshar K (2013) Application of IPSO-Monte Carlo for optimal distributed generation allocation and sizing. Int J Electr Power Energy Syst 44:786-797. doi: 10.1016/j.ijepes.2012.08.006

Abul'Wafa AR (2016) Multi-conductor feeder design for radial distribution networks. Electr Power Syst Res 140:184-192. doi: 10.1016/j.epsr.2016.06.023

Acharya N, Mahat P, Mithulananthan N (2006) An analytical approach for DG allocation in primary distribution network. Int J Electr Power Energy Syst 28:669-678. doi: 10.1016/j.ijepes.2006.02.013

Almasri R (2011) Advanced CSP Teaching Materials Chapter 13 Electrical Power Transmission Author.

Alrashidi MR, Alhajri MF (2011) Optimal planning of multiple distributed generation sources in distribution networks: A new approach. Energy Convers Manag 52:3301-3308. doi: 10.1016/j.enconman.2011.06.001

Aman MM, Jasmon GB, Bakar AHA, Mokhlis H (2014) A new approach for optimum simultaneous multi-DG distributed generation Units placement and sizing based on maximization of system loadability using HPSO (hybrid particle swarm optimization) algorithm. Energy 66:202-215. doi: 10.1016/j.energy.2013.12.037

Aman MM, Jasmon GB, Mokhlis H, Bakar AHA (2012) Optimal placement and sizing of a DG based on a new power stability index and line losses. Int J Electr Power Energy Syst 43:1296-1304. doi: 10.1016/j.ijepes.2012.05.053

Andervazh M-R, Haghifam M-R, Olamaei J (2013) Adaptive multi-objective distribution network reconfiguration using multiobjective discrete particles swarm optimisation algorithm and graph theory. IET Gener Transm Distrib 7:1367-1382. doi: 10.1049/iet-gtd.2012.0712

Ayres HM, Freitas W, De Almeida MC, Da Silva LCP (2010) Method for determining the maximum allowable penetration level of distributed generation without steady-state voltage violations. IET Gener Transm Distrib 4:495. doi: 10.1049/iet-gtd.2009.0317

Azizipanah-Abarghooee R, Javidsharifi M, Narimani MR, Azizi Vahed A (2014) Enhanced gravitational search algorithm for multiobjective distribution feeder reconfiguration considering reliability, loss and operational cost. IET Gener Transm Distrib 8:5569. doi: 10.1049/iet-gtd.2013.0117

Bagheri Tolabi H, Ali MH, Rizwan M (2015) Simultaneous reconfiguration, optimal placement of DSTATCOM, and photovoltaic array in a distribution system based on fuzzy-aco approach. IEEE Trans Sustain Energy 6:210-218. doi: 10.1109/TSTE.2014.2364230

Baghipourr R, Hosseini SM (2013) Optimal Placement and Sizing of Distributed Generation And Capacitor Bank For Loss Reduction And Reliability Improvement In Distribution Systems. Majlesi J Electr Eng 7:58-65. doi: 10.1016/j.egypro.2013.06.759

Baghzouz Y, Ertem S (1990) Shunt capacitor sizing for radial distribution feeders with distorted substation voltages. IEEE Trans Power Deliv 5:650-657. doi: 10.1109/61.53067

Bakhshideh Zad B, Hasanvand H, Lobry J, Vallée F (2015) Optimal reactive power control of DGs for voltage regulation of MV distribution systems using sensitivity analysis method and PSO algorithm. Int J Electr Power Energy Syst 68:52-60. doi: 10.1016/j.ijepes.2014.12.046

Baran ME, Wu FF (1989a) Network reconfiguration in distribution systems for loss reduction and load balancing. Power Deliv IEEE Trans 4:1401-1407. doi: 10.1109/61.25627

Baran ME, Wu FF (1989b) Optimal sizing of capacitors placed on a radial distribution system. IEEE Trans Power Deliv 4:735-743. 
doi: $10.1109 / 61.19266$

Baran ME, Wu FF (1989c) Optimal capacitor placement on radial distribution systems. IEEE Trans Power Deliv 4:725-734. doi: $10.1109 / 61.19265$

Battu NR, Abhyankar AR, Senroy N (2016) DG Planning with Amalgamation of Operational and Reliability Considerations. Int J Emerg Electr Power Syst 17:131-141. doi: 10.1515/ijeeps-2015-0142

Bhongade S, Arya S (2016) Optimal Capacitors in Radial Distribution System for Loss Reduction and Voltage Enhancement. Indones J Electr Eng Comput Sci 2:566-582. doi: 10.11591/ijeecs.v2.i2.pp566-582

Capitanescu F, Ochoa LF, Margossian H, Hatziargyriou ND (2015) Assessing the potential of network reconfiguration to improve distributed generation hosting capacity in active distribution systems. IEEE Trans Power Syst 30:346-356. doi: 10.1109/TPWRS.2014.2320895

ChithraDevi SA, Lakshminarasimman L, Balamurugan R (2016) Stud Krill herd Algorithm for multiple DG placement and sizing in a radial distribution system. Eng Sci Technol an Int J. doi: 10.1016/j.jestch.2016.11.009

Civanlar S, Grainger JJ, Yin H, Lee SSH (1988) Distribution Feeder Reconfiguration for Loss Reduction. IEEE Trans Power Deliv 3:1217-1223. doi: 10.1109/61.193906

Das D (1994) Novel method for solving radial distribution networks. IEE Proc - Gener Transm Distrib 141:291. doi: 10.1049/ipgtd:19949966

Das D (2006) Reconfiguration of distribution system using fuzzy multi-objective approach. Int J Electr Power Energy Syst 28:331338. doi: 10.1016/j.ijepes.2005.08.018

Das D, Kothari DP, Kalam A (1995) Simple and efficient method for load flow solution of radial distribution networks. Int J Electr Power Energy Syst 17:335-346. doi: 10.1016/0142-0615(95)00050-0

Devabalaji KR, Ravi K, Kothari DP (2015) Optimal location and sizing of capacitor placement in radial distribution system using Bacterial Foraging Optimization Algorithm. Int J Electr Power Energy Syst 71:383-390. doi: 10.1016/j.ijepes.2015.03.008

Devi S, Geethanjali M (2014) Optimal location and sizing determination of Distributed Generation and DSTATCOM using Particle Swarm Optimization algorithm. Int J Electr Power Energy Syst 62:562-570. doi: 10.1016/j.ijepes.2014.05.015

Diab AAZ, Tulsky VN, Tolba MA (2017) Optimal shunt capacitors sittings and sizing in radial distribution systems using a novel hybrid optimization algorithm. 2016 18th Int Middle-East Power Syst Conf MEPCON 2016 - Proc. doi: 10.1109/MEPCON.2016.7836929

Dias FM, Canizes B, Khodr H, Cordeiro M (2015) Distribution networks planning using decomposition optimisation technique. IET Gener Transm Distrib 9:1409-1420. doi: 10.1049/iet-gtd.2014.0860

Dixit M, Kundu P, Jariwala HR (2017) Incorporation of distributed generation and shunt capacitor in radial distribution system for techno-economic benefits. Eng Sci Technol an Int J 20:482-493. doi: 10.1016/j.jestch.2017.01.003

Ebrahimi R, Ehsan M, Nouri H (2013) U-shaped energy loss curves utilization for distributed generation optimization in distribution networks. J Zhejiang Univ Sci C 14:887-898. doi: 10.1631/jzus.C1200282

El-Fergany A (2015) Study impact of various load models on DG placement and sizing using backtracking search algorithm. Appl Soft Comput J 30:803-811. doi: 10.1016/j.asoc.2015.02.028

El Ramli R, Awad M, Jabr RA (2011) Ordinal Optimization for Dynamic Network Reconfiguration. Electr Power Components Syst 39:1845-1857. doi: 10.1080/15325008.2011.615801

Elsheikh A, Helmy Y, Abouelseoud Y, Elsherif A (2014) Optimal capacitor placement and sizing in radial electric power systems. Alexandria Eng J 53:809-816. doi: 10.1016/j.aej.2014.09.012

Esmaeilian HR, Fadaeinedjad R (2014) Energy Loss Minimization in Distribution Systems Utilizing an Enhanced Reconfiguration Method Integrating Distributed Generation. IEEE Syst J 1-10. doi: 10.1109/JSYST.2014.2341579

Ettehadi M, Ghasemi H, Vaez-Zadeh S (2013) Voltage stability-based DG placement in distribution networks. IEEE Trans Power Deliv 28:171-178. doi: 10.1109/TPWRD.2012.2214241

Gampa SR, Das D (2015) Optimum placement and sizing of DGs considering average hourly variations of load. Int J Electr Power Energy Syst 66:25-40. doi: 10.1016/j.ijepes.2014.10.047

Gampa SR, Das D (2016) Optimum placement of shunt capacitors in a radial distribution system for substation power factor improvement using fuzzy GA method. Int J Electr Power Energy Syst 77:314-326. doi: 10.1016/j.ijepes.2015.11.056

García JAM, Mena AJG (2013) Optimal distributed generation location and size using a modified teaching-learning based optimization algorithm. Int J Electr Power Energy Syst 50:65-75. doi: 10.1016/j.ijepes.2013.02.023

Georgilakis PS, Hatziargyriou ND (2013) Optimal distributed generation placement in power distribution networks: Models, methods, and future research. IEEE Trans Power Syst 28:3420-3428. doi: 10.1109/TPWRS.2012.2237043

Gopiya Naik S, Khatod DK, Sharma MP (2013) Optimal allocation of combined DG and capacitor for real power loss minimization in distribution networks. Int J Electr Power Energy Syst 53:967-973. doi: 10.1016/j.ijepes.2013.06.008

Gözel T, Eminoglu U, Hocaoglu MH (2008) A tool for voltage stability and optimization (VS\&OP) in radial distribution systems 
using matlab graphical user interface (GUI). Simul Model Pract Theory 16:505-518. doi: 10.1016/j.simpat.2008.02.003

Gözel T, Hocaoglu MH (2009) An analytical method for the sizing and siting of distributed generators in radial systems. Electr Power Syst Res 79:912-918. doi: 10.1016/j.epsr.2008.12.007

Gupta N, Swarnkar A, Bansal RC, Niazi KR (2010) Multi-objective reconfiguration of distribution systems using adaptive genetic algorithm in fuzzy framework. IET Gener Transm Distrib 4:1288-1298. doi: 10.1049/iet-gtd.2010.0056

Hamouda A, Sayah S (2013) Optimal capacitors sizing in distribution feeders using heuristic search based node stability-indices. Int J Electr Power Energy Syst 46:56-64. doi: 10.1016/j.ijepes.2012.10.016

Hamouda A, Zehar K (2011) Stability-index based method for optimal Var planning in distribution feeders. Energy Convers Manag 52:2072-2080. doi: 10.1016/j.enconman.2010.12.002

Hamouda A, Zehar K (2006) Efficient load flow method for radial distribution feeders. J. Appl. Sci. 6:2741-2748.

Hemmatpour MH, Mohammadian M (2013) An Evolutionary Approach for Optimum Reconfiguration and Distributed Generation Planning Considering Variable Load Pattern Based on Voltage Security Margin. Arab J Sci Eng 38:3407-3420. doi: 10.1007/s13369-013-0623-0

Hengsritawat V, Tayjasanant T, Nimpitiwan N (2012) Optimal sizing of photovoltaic distributed generators in a distribution system with consideration of solar radiation and harmonic distortion. Int J Electr Power Energy Syst 39:36-47. doi: 10.1016/j.ijepes.2012.01.002

HLAING CS, SWE PL (2014) Sizing and Sitting of Distributed Generation in Distribution System for Loss Minimization and Voltage Stability. Int J Sci Eng Technol Res 3:9148-9153.

Hung DQ, Mithulananthan N (2013) Multiple distributed generator placement in primary distribution networks for loss reduction. IEEE Trans Ind Electron 60:1700-1708. doi: 10.1109/TIE.2011.2112316

Hung DQ, Mithulananthan N, Bansal RC (2010) Analytical expressions for DG allocation in primary distribution networks. IEEE Trans Energy Convers 25:814-820. doi: 10.1109/TEC.2010.2044414

Injeti SK, Prema Kumar N (2013) A novel approach to identify optimal access point and capacity of multiple DGs in a small, medium and large scale radial distribution systems. Int J Electr Power Energy Syst 45:142-151. doi: 10.1016/j.ijepes.2012.08.043

Javadi MS, Esmaeel Nezhad A, Siano P, et al (2017) Shunt capacitor placement in radial distribution networks considering switching transients decision making approach. Int J Electr Power Energy Syst 92:167-180. doi: 10.1016/j.jjepes.2017.05.001

Kansal S, Kumar V, Tyagi B (2013) Optimal placement of different type of DG sources in distribution networks. Int J Electr Power Energy Syst 53:752-760. doi: 10.1016/j.ijepes.2013.05.040

Kansal S, Kumar V, Tyagi B (2016) Hybrid approach for optimal placement of multiple DGs of multiple types in distribution networks. Int J Electr Power Energy Syst 75:226-235. doi: 10.1016/j.jepes.2015.09.002

Kanwar N, Gupta N, Niazi KR, Swarnkar A (2015) Improved meta-heuristic techniques for simultaneous capacitor and DG allocation in radial distribution networks. Int J Electr Power Energy Syst 73:653-664. doi: 10.1016/j.ijepes.2015.05.049

Kashem MA, Ganapathy V, Jasmon GB (2001) A geometrical approach for network reconfiguration based loss minimization in distribution systems. Electr Power Energy Syst 23:295-304.

Kayal P, Chanda CK (2013) Placement of wind and solar based DGs in distribution system for power loss minimization and voltage stability improvement. Int J Electr Power Energy Syst 53:795-809. doi: 10.1016/j.ijepes.2013.05.047

Khan NA, Ghosh S, Ghoshal SP (2013) Optimum Siting and Sizing of Shunt Capacitors in Radial Distribution System using Novel BPSO Algorithm. IEE Proc Gener TransmDistrib 3:220-225. doi: 10.4236/epe.2013.54B192

Lee C, Liu C, Mehrotra S, Bie Z (2014) Robust Distribution Network Reconfiguration. 6:1-7.

Lee SH, Park JW (2009) Selection of optimal location and size of multiple distributed generations by using Kalman Filter algorithm. IEEE Trans Power Syst 24:1393-1400. doi: 10.1109/TPWRS.2009.2016540

Mantovani JRS, Casari F, Romero RA (2000) Reconfiguração de sistemas de distribuição radiais utilizando o critério de queda de tensão. Rev Bras Control Automação - SBA 11:150-159.

Masoum MAS, Ladjevardi M, Jafarian A, Fuchs EF (2004) Optimal placement, replacement and sizing of capacitor banks in distorted distribution networks by genetic algorithms. IEEE Trans Power Deliv 19:1794-1801. doi: 10.1109/TPWRD.2004.835438

Mena AJG, García JAM (2015) An efficient approach for the siting and sizing problem of distributed generation. Int J Electr Power Energy Syst 69:167-172. doi: 10.1016/j.ijepes.2015.01.011

Mendoza J, Morales D, López R, et al (2007) Multi-objective Location of Automatic Voltage Regulators in a Radial Distribution Network Using a Micro Genetic Algorithm. IEEE Trans Power Syst 22:404-411.

Mohamed Imran A, Kowsalya M, Kothari DP (2014) A novel integration technique for optimal network reconfiguration and distributed generation placement in power distribution networks. Int J Electr Power Energy Syst 63:461-472. doi: 10.1016/j.ijepes.2014.06.011

Mohandas N, Balamurugan R, Lakshminarasimman L (2015) Optimal location and sizing of real power DG units to improve the voltage stability in the distribution system using ABC algorithm united with chaos. Int J Electr Power Energy Syst 66:41-52. 
doi: 10.1016/j.ijepes.2014.10.033

Moradi MH, Abedini M (2012) A combination of genetic algorithm and particle swarm optimization for optimal DG location and sizing in distribution systems. Int J Electr Power Energy Syst 34:66-74. doi: 10.1016/j.ijepes.2011.08.023

Moradi MH, Zeinalzadeh A, Mohammadi Y, Abedini M (2014) An efficient hybrid method for solving the optimal sitting and sizing problem of DG and shunt capacitor banks simultaneously based on imperialist competitive algorithm and genetic algorithm. Int J Electr Power Energy Syst 54:101-111. doi: 10.1016/j.ijepes.2013.06.023

Murthy KVSR, Bhimaraju PSD (2016) Optimal Sizing of Capacitors Using Harmony Search Algorithm with Limited Search Space. Int J Emerg Trends Electr Electron 12:1-10.

Muthukumar R, Thanushkodi K (2013) Opposition based differential evolution algorithm for capacitor placement on radial distribution system. J Electr Eng Technol 8:742-748.

Myint SM (2015) Analysis of Loss Reduction for Radial Distribution System Using Network Reconfiguration. Int J Eng Tech 1:686690. doi: 10.17148/IJARCCE.2015.45144

Myint SM, Naing SW (2015) Network Reconfiguration for Loss Reduction and Voltage Stability Improvement of 74-bus Radial Distribution System Using Particle Swarm Optimization Algorithm.

Nekooei K, Farsangi MM, Nezamabadi-Pour H, Lee KY (2013) An improved multi-objective harmony search for optimal placement of DGs in distribution systems. IEEE Trans Smart Grid 4:557-567. doi: 10.1109/TSG.2012.2237420

Niknam T, Narimani MR, Azizipanah-Abarghooee R (2012) A multi-objective fuzzy adaptive pso algorithm for location of automatic voltage regulators in radial distribution networks. Int J Control Autom Syst 10:772-777. doi: 10.1007/s12555-012-0413-6

Nojavan S, Jalali M, Zare K (2014) Optimal allocation of capacitors in radial/mesh distribution systems using mixed integer nonlinear programming approach. Electr Power Syst Res 107:119-124. doi: 10.1016/j.epsr.2013.09.019

Pazouki S, Mohsenzadeh A, Member S (2015) Simultaneous Planning of PEV Charging Stations and DGs Considering Financial, Technical , and Environmental Effects. Can J Electr Comput Eng 38:238-245. doi: 10.1109/CJECE.2015.2436811

Peddanna G (2015) Power Factor and Energy Loss Cost Evaluation of Radial Distribution Systems. Int J Adv Res Electr Electron Instrum Eng 4:7544-7548. doi: 10.15662/IJAREEIE.2015.0409021

Peddanna G, Kishore YSR (2015) Power Loss Allocation of Balanced Radial Distribution Systems. Int J Sci Res 4:360-366.

Pires DF, Antunes CH, Martins AG (2012) NSGA-II with local search for a multi-objective reactive power compensation problem. Int J Electr Power Energy Syst 43:313-324. doi: 10.1016/j.ijepes.2012.05.024

Rajaram R, Sathish Kumar K, Rajasekar N (2015) Power system reconfiguration in a radial distribution network for reducing losses and to improve voltage profile using modified plant growth simulation algorithm with Distributed Generation (DG). Energy Reports 1:116-122. doi: 10.1016/j.egyr.2015.03.002

Ramalinga Raju M, Ramachandra Murthy KVS, Ravindra K (2012) Direct search algorithm for capacitive compensation in radial distribution systems. Int J Electr Power Energy Syst 42:24-30. doi: 10.1016/j.ijepes.2012.03.006

Rao RS, Narasimham SVL (2008) Optimal capacitor placement in a radial distribution system using Plant Growth Simulation Algorithm. World Acad Sci Eng Technol 21:715-722. doi: 10.1016/j.ijepes.2010.11.021

Rao RS, Satish K, Narasimham SVL (2012) Optimal Conductor Size Selection in Distribution Systems Using the Harmony Search Algorithm with a Differential Operator. Electr Power Components Syst 40:41-56. doi: 10.1080/15325008.2011.621922

Raoofat M (2011) Simultaneous allocation of DGs and remote controllable switches in distribution networks considering multilevel load model. Int J Electr Power Energy Syst 33:1429-1436. doi: 10.1016/j.ijepes.2011.06.023

Reddy PDP, Reddy VCV, Manohar TG (2017) Whale optimization algorithm for optimal sizing of renewable resources for loss reduction in distribution systems. Renewables Wind Water, Sol 4:3. doi: 10.1186/s40807-017-0040-1

Rosseti GJS, De Oliveira EJ, De Oliveira LW, et al (2013) Optimal allocation of distributed generation with reconfiguration in electric distribution systems. Electr Power Syst Res 103:178-183. doi: 10.1016/j.epsr.2013.05.017

S. Vijayabaskar, T. Manigandan (2013) Analysis of Radial Distribution System Optimization With FACTS Devices Using Hybrid Heuristic Technique. J Theor Appl Inf Technol 58:397-404.

Salama MMA, Chikhani AY (1993) Simplified network approach to the var control problem for radial distribution systems. IEEE Trans Power Deliv 8:1529-1535. doi: 10.1109/61.252679

Shaaban MF, Atwa YM, El-Saadany EF (2013) DG allocation for benefit maximization in distribution networks. IEEE Trans Power Syst 28:939-949. doi: 10.1109/TPWRS.2012.2213309

Shukla TN, Singh SP, Srinivasarao V, Naik KB (2010) Optimal Sizing of Distributed Generation Placed on Radial Distribution Systems. Electr Power Components Syst 38:260-274. doi: 10.1080/15325000903273403

Singh D, Misra RK (2007) Effect of Load Models in Distributed Generation Planning. Power Syst IEEE Trans 22:2204-2212. doi: 10.1109/TPWRS.2007.907582

Sultana S, Roy PK (2015) Oppositional krill herd algorithm for optimal location of distributed generator in radial distribution system. Electr Power Energy Syst 73:182-191. doi: 10.1016/j.asoc.2015.11.036 
Sultana S, Roy PK (2016) Krill herd algorithm for optimal location of distributed generator in radial distribution system. Appl Soft Comput J 40:391-404. doi: 10.1016/j.asoc.2015.11.036

Sultana S, Roy PK (2014a) Multi-objective quasi-oppositional teaching learning based optimization for optimal location of distributed generator in radial distribution systems. Int J Electr Power Energy Syst 63:534-545. doi: 10.1016/j.ijepes.2014.06.031

Sultana S, Roy PK (2014b) Optimal capacitor placement in radial distribution systems using teaching learning based optimization. Int J Electr Power Energy Syst 54:387-398. doi: 10.1016/j.ijepes.2013.07.011

V.K. Mehta and R. Mehta (2011) Principles of Power System.

Wang C, Nehrir MH (2004) Analytical approaches for optimal placement of distributed generation sources in power systems. IEEE Trans Power Syst 19:2068-2076. doi: 10.1109/TPWRS.2004.836189

Warren CA (2003) IEEE Guide for Electric Power Distribution Reliability Indices.

Xavier F, Llavall B (2011) Reliability Worth Assessment of Radial System With Distributed Generation Illinois Institute of Technology.

Yuvaraj T, Ravi K, Devabalaji KR (2017) Optimal Allocation of DG and DSTATCOM in Radial Distribution System Using Cuckoo Search Optimization Algorithm. Model Simul Eng. doi: 10.1155/2017/2857926

Zare K, Abapour S, Mohammadi-Ivatloo B (2015) Dynamic planning of distributed generation units in active distribution network. IET Gener Transm Distrib 9:1455-1463. doi: 10.1049/iet-gtd.2014.1143

Zhang D, Fu Z, Zhang L (2007) An improved TS algorithm for loss-minimum reconfiguration in large-scale distribution systems. Electr Power Syst Res 77:685-694. doi: 10.1016/j.epsr.2006.06.005

Zhang L, Tang W, Liu Y, Lv T (2015) Multiobjective optimization and decision-making for DG planning considering benefits between distribution company and DGs owner. Int J Electr Power Energy Syst 73:465-474. doi: 10.1016/j.ijepes.2015.05.019

Zhu D, Broadwater RP, Tam KS, et al (2006) Impact of DG placement on reliability and efficiency with time-varying loads. IEEE Trans Power Syst 21:419-427. doi: 10.1109/TPWRS.2005.860943

Zhu JZ (2002) Optimal reconfiguration of electrical distribution network using the refined genetic algorithm. Electr Power Syst Res 62:37-42. doi: 10.1016/S0378-7796(02)00041-X

Zimmerman RD, Murillo-Sanchez CE, Thomas RJ (2011) Matpower: Steady- State Operations, Planning and Analysis Tools for Power Systems Research and Education. IEEE Trans Power Syst 26:12-19. 